\title{
On the Relationship Between the Preferred Site of Hydrogen Bonding and Protonation
}

\author{
Bun Chan,, ${ }^{* \dagger}$ Janet E. Del Bene,, ${ }^{*, \neq}$ Jose Elguero, ${ }^{*, \uparrow}$ and Leo \\ Radom*,† \\ Contribution from the School of Chemistry, University of Sydney, Sydney, \\ NSW 2006, Australia, Department of Chemistry, Youngstown State \\ University, Youngstown, Ohio 44555, USA, and Instituto de Quimica \\ Medica, CSIC, Juan de la Cierva, 3, E-28006 Madrid, Spain \\ (E-mail: chan_b@chem.usyd.edu.au; jedelbene@ysu.edu; \\ iqmbe17@iqm.csic.es; radom@chem.usyd.edu.au)
}

\section{Supporting Information}

(Tables S1-S5, Ref 16, Total 29 pages) 
Table S1. GAUSSIAN Archive Entries for the MP2/6-31+G(d,p) Optimized Geometries

\begin{abstract}
$\mathrm{C}_{3} \mathrm{H}_{3}^{-}$(Cyclopropenyl Anion)

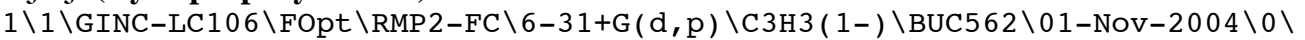

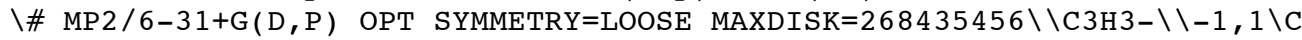
$, 0 .,-0.1245035389,0.9745260837 \backslash \mathrm{C},-0.6576393617,0.0295333019,-0.4360614$ $867 \backslash \mathrm{C}, 0.6576393617,0.0295333019,-0.4360614867 \backslash \mathrm{H},-1.5155435971,-0.23745$ $50933,-1.0408966576 \backslash \mathrm{H}, 1.5155435971,-0.2374550933,-1.0408966576 \backslash \mathrm{H}, 0 ., 0$. $8675317972,1.4673746535 \backslash \backslash$ Version=x86-Linux-G03RevB.03 $\backslash$ State $=1-A^{\prime} \backslash \mathrm{HF}=-1$ $15.1323139 \backslash \mathrm{MP} 2=-115.555326 \backslash \mathrm{RMSD}=7.541 \mathrm{e}-09 \backslash \mathrm{RMSF}=2.318 \mathrm{e}-05 \backslash \mathrm{Dipole}=0 ., 0.6$ $854174,-1.8358607 \backslash \mathrm{PG}=\mathrm{CS}[\mathrm{SG}(\mathrm{C} 1 \mathrm{H} 1), \mathrm{X}(\mathrm{C} 2 \mathrm{H} 2)] \backslash \backslash @$
\end{abstract}

$\mathrm{C}_{3} \mathbf{H}_{4}$

$1 \backslash 1 \backslash$ GINC-LC2 $\backslash$ FOpt $\backslash$ RMP $2-F C \backslash 6-31+G(d, p) \backslash C 3 H 4 \backslash B U C 562 \backslash 01-N o v-2004 \backslash 0 \backslash \backslash \#$ MP2

$/ 6-31+G(D, P)$ OPT SYMMETRY=LOOSE MAXDISK $=268435456 \backslash \backslash \mathrm{C} 3 \mathrm{H} 4 \backslash \backslash 0,1 \backslash \mathrm{C}, 0,0,0$ $. \backslash \mathrm{C}, 0 .,-0.6527,-1.361592 \backslash \mathrm{C}, 0 ., 0.6527,-1.361592 \backslash \mathrm{H}, 0 .,-1.5814,-1.901892 \backslash$ $\mathrm{H}, 0 ., 1.5814,-1.901892 \backslash \mathrm{H},-0.911,0 ., 0.591108 \backslash \mathrm{H}, 0.911,0 ., 0.591108 \backslash \backslash$ Versio $\mathrm{n}=\mathrm{x} 86-\mathrm{Linux}-\mathrm{G} 03$ RevB $.03 \backslash$ State $=1-\mathrm{A} 1 \backslash \mathrm{HF}=-115.8327386 \backslash \mathrm{MP} 2=-116.2438916 \backslash \mathrm{RMS}$ $\mathrm{D}=4.793 \mathrm{e}-09 \backslash \mathrm{RMSF}=1.523 \mathrm{e}-04 \backslash \mathrm{Dipole}=0,0 .,-0.1681107 \backslash \mathrm{PG}=\mathrm{C} 02 \mathrm{~V} \quad[\mathrm{C} 2(\mathrm{C} 1), \mathrm{SGV}$ ( $\left.\mathrm{C} 2 \mathrm{H} 2), \mathrm{SGV}^{\prime}(\mathrm{H} 2)\right] \backslash \backslash @$

\title{
$\mathrm{C}_{3} \mathrm{H}_{3}^{-\cdots \cdots} \mathrm{HF}(\mathrm{C1})$
}

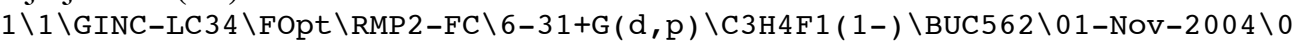

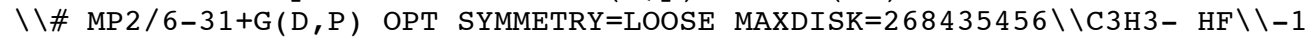
$, 1 \backslash \mathrm{C}, 0 .,-0.6583467115,0.3828950519 \backslash \mathrm{C},-0.6544339084,0.2673484523,1.3950$ $405162 \backslash \mathrm{C}, 0.6544339084,0.2673484523,1.3950405162 \backslash \mathrm{H},-1.5697646186,0.7251$ $304144,1.7283255623 \backslash \mathrm{H}, 1.5697646186,0.7251304144,1.7283255623 \backslash \mathrm{H}, 0 .,-1.7$ $240423113,0.6166323596 \backslash \mathrm{H}, 0 .,-0.3889359708,-0.6833610329 \backslash \mathrm{F}, 0 ., 0.1560684$ $771,-2.4919754397 \backslash \backslash$ Version $=x 86-$ Linux $^{\prime}$ G03RevB.03 $\backslash$ State $=1-A^{\prime} \backslash \mathrm{HF}=-215.258$ $2122 \backslash M P 2=-215.878047 \backslash \mathrm{RMSD}=7.413 e-09 \backslash \mathrm{RMSF}=1.376 \mathrm{e}-04 \backslash \mathrm{Dipole}=0 .,-0.113315$ $2,3.8961424 \backslash \mathrm{PG}=\mathrm{CS}[\mathrm{SG}(\mathrm{C} 1 \mathrm{H} 2 \mathrm{~F} 1), \mathrm{X}(\mathrm{C} 2 \mathrm{H} 2)] \backslash \backslash @$

\section{$\mathrm{C}_{3} \mathrm{H}_{3}^{-} \bullet \bullet \cdot \mathrm{HF}(\mathrm{C}=\mathrm{C})$}

$1 \backslash 1 \backslash$ GINC-LC1 $15 \backslash F O p t \backslash R M P 2-F C \backslash 6-31+G(d, p) \backslash C 3 H 4 F 1(1-) \backslash B U C 562 \backslash 01-N o v-2004 \backslash$

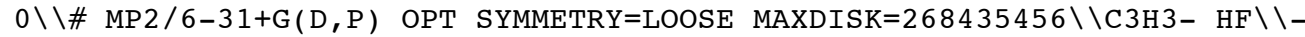
$1,1 \backslash \mathrm{C},-0.6556167468,-0.6578437363,-0.3622688724 \backslash \mathrm{C},-0.6556167468,0.6578$ $437363,-0.3622688724 \backslash \mathrm{C},-1.6864714004,0.0 .6272393301 \backslash \mathrm{H},-0.1054594816,-$ $1.5511448952,-0.62761934 \backslash \mathrm{H},-0.1054594816,1.5511448952,-0.62761934 \backslash \mathrm{H},-2$ $.6660576457,0 ., 0.1035722639 \backslash \mathrm{H}, 1.2297354488,0 ., 0.1008997152 \backslash \mathrm{F}, 2.1814967$ $249,0 ., 0.1816174654 \backslash \backslash$ Version $=x 86-L$ inux-G03RevB.03 $\backslash$ State $=1-A^{\prime} \backslash \mathrm{HF}=-215.1$ $757564 \backslash M P 2=-215.7951669 \backslash \mathrm{RMSD}=6.252 \mathrm{e}-09 \backslash \mathrm{RMSF}=9.970 \mathrm{e}-05 \backslash \mathrm{Dipole}=1.5382197$ , $0 .,-1.6897967 \backslash \mathrm{PG}=\mathrm{CS}[\mathrm{SG}(\mathrm{C} 1 \mathrm{H} 2 \mathrm{~F} 1), \mathrm{X}(\mathrm{C} 2 \mathrm{H} 2)] \backslash \backslash @$

\section{$\mathrm{C}_{4} \mathrm{H}_{4}$ (Cyclobutadiene)}

$1 \backslash 1 \backslash$ GINC-LC3 \FOpt \RMP2-FC $\backslash 6-31+\mathrm{G}(\mathrm{d}, \mathrm{p}) \backslash \mathrm{C} 4 \mathrm{H} 5 \mathrm{~F} 1 \backslash \mathrm{BUC5} 62 \backslash 01-\mathrm{Nov}-2004 \backslash 0 \backslash \backslash \# \mathrm{M}$ P2 /6-31+G(D,P) OPT SYMMETRY=LOOSE MAXDISK $=268435456 \backslash \backslash \mathrm{C} 4 \mathrm{H} 4-\mathrm{HF} \backslash \backslash 0,1 \backslash \mathrm{C}$, $-0.3388391986,-0.676236649,0.6912821636 \backslash C,-1.415806678,-0.6744593982,-$ $0.4435467916 \backslash \mathrm{C},-1.415806678,0.6744593982,-0.4435467916 \backslash \mathrm{C},-0.3388391986$ $, 0.676236649,0.6912821636 \backslash \mathrm{H}, 0.1370562592,-1.4392670019,1.2904351581 \backslash \mathrm{H}$, $-1.9213294796,-1.4416208272,-1.0098753163 \backslash \mathrm{H},-1.9213294796,1.4416208272$ $,-1.0098753163 \backslash \mathrm{H}, 0.1370562592,1.4392670019,1.2904351581 \backslash \mathrm{H}, 1.6607194597$ $, 0 .,-0.0920700081 \backslash \mathrm{F}, 2.5515086112,0 .,-0.38243046 \backslash \backslash$ Version $=x 86-$ Linux-G03 RevB. 03 \State $=1-A^{\prime} \backslash \mathrm{HF}=-253.6821374 \backslash \mathrm{MP} 2=-254.4126241 \backslash \mathrm{RMSD}=4.011 \mathrm{e}-09 \backslash \mathrm{RMS}$ $\mathrm{F}=1.672 \mathrm{e}-04 \backslash \mathrm{Dipole}=-1.0778671,0 ., 0.322937 \backslash \mathrm{PG}=\mathrm{CS} \quad[\mathrm{SG}(\mathrm{H} 1 \mathrm{~F} 1), \mathrm{X}(\mathrm{C} 4 \mathrm{H} 4)] \backslash \backslash @$

\section{$\mathrm{C}_{4} \mathrm{H}_{5}^{+}(\mathrm{C} 1)$}

$1 \backslash 1 \backslash G I N C-L C 138 \backslash F O p t \backslash R M P 2-F C \backslash 6-31+G(d, p) \backslash C 4 H 5(1+) \backslash B U C 562 \backslash 01-N o v-2004 \backslash 0 \backslash$ $\backslash \#$ MP2 $/ 6-31+G(D, P)$ OPT SYMMETRY $=$ LOOSE MAXDISK $=268435456 \backslash \backslash \mathrm{C} 4 \mathrm{H} 5+\backslash \backslash 1,1 \backslash \mathrm{C}$, $0 ., 0 ., 0 . \backslash C,-1.186,0.7542739093,-0.5396632374 \backslash C,-1.186,-0.9237210771,-0$ $.0830957989 \backslash \mathrm{C},-2.205699,0.0138653151,0.050958363 \backslash \mathrm{H},-1.24,1.3688313333$, $-1.432846126 \backslash \mathrm{H},-1.24,-1.9061054025,-0.5417651103 \backslash \mathrm{H}, 0.84437,-0.17880483$ $12,-0.6571506966 \backslash \mathrm{H}, 0.270182,0.2753324818,1.0119129949 \backslash \mathrm{H},-3.283544,0.01$ $91800332,0.0704912281 \backslash \backslash$ Version $=x 86-$ Linux-G03RevB.03 $\backslash$ State $=1-A^{\prime} \backslash \mathrm{HF}=-154$ $.0503789 \backslash M P 2=-154.5686243 \backslash \mathrm{RMSD}=5.491 \mathrm{e}-09 \backslash \mathrm{RMSF}=2.516 \mathrm{e}-04 \backslash \mathrm{Dipole}=0,,-0.3$ $34081,-0.2824033 \backslash P G=C S[S G(C 2 H 3), X(C 2 H 2)] \backslash \backslash @$ 


\section{$\mathrm{C}_{4} \mathrm{H}_{4} \cdots \cdot \mathrm{HF}(\mathrm{C}=\mathrm{C})$}

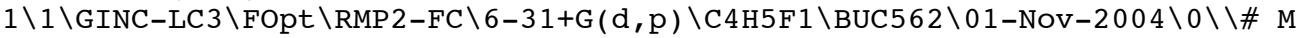
P2 $/ 6-31+G(D, P)$ OPT SYMMETRY=LOOSE MAXDISK $=268435456 \backslash \backslash \mathrm{C} 4 \mathrm{H} 4-\mathrm{HF} \backslash \backslash 0,1 \backslash \mathrm{C}$, $-0.3388391986,-0.676236649,0.6912821636 \backslash C,-1.415806678,-0.6744593982,-$ $0.4435467916 \backslash \mathrm{C},-1.415806678,0.6744593982,-0.4435467916 \backslash \mathrm{C},-0.3388391986$ $, 0.676236649,0.6912821636 \backslash \mathrm{H}, 0.1370562592,-1.4392670019,1.2904351581 \backslash \mathrm{H}$, $-1.9213294796,-1.4416208272,-1.0098753163 \backslash \mathrm{H},-1.9213294796,1.4416208272$ $,-1.0098753163 \backslash \mathrm{H}, 0.1370562592,1.4392670019,1.2904351581 \backslash \mathrm{H}, 1.6607194597$ $, 0 .,-0.0920700081 \backslash \mathrm{F}, 2.5515086112,0 .,-0.38243046 \backslash \backslash$ Version $=x 86-\mathrm{Linux}-\mathrm{G} 03$ RevB.03 $\backslash$ State $=1-A^{\prime} \backslash \mathrm{HF}=-253.6821374 \backslash \mathrm{MP} 2=-254.4126241 \backslash \mathrm{RMSD}=4.011 \mathrm{e}-09 \backslash \mathrm{RMS}$ $\mathrm{F}=1.672 \mathrm{e}-04 \backslash \mathrm{Dipole}=-1.0778671,0 ., 0.322937 \backslash \mathrm{PG}=\mathrm{CS} \quad[\mathrm{SG}(\mathrm{H} 1 \mathrm{~F} 1), \mathrm{X}(\mathrm{C} 4 \mathrm{H} 4)] \backslash \backslash @$

\section{$\mathrm{NH}_{2} \mathrm{CHNH}$ (Formamidine)}

$1 \backslash 1 \backslash G I N C-S C 2 \backslash F O p t \backslash R M P 2-F C \backslash 6-31+G(d, p) \backslash C 1 H 4 N 2 \backslash B U C 562 \backslash 25-O C t-2004 \backslash 0 \backslash \backslash \# M$ $\mathrm{P} 2 / 6-31+\mathrm{G}(\mathrm{D}, \mathrm{P}) \quad \mathrm{OPT}$ MAXDISK $=268435456 \backslash \backslash \mathrm{NHCHNH} 2 \backslash \backslash 0,1 \backslash \mathrm{C},-0.3523228591,0.2$ $216932462,0.0346815008 \backslash \mathrm{N}, 0.639180731,1.0419516166,0.0085739926 \backslash \mathrm{N},-0.16$ $70929378,-1.1345888494,-0.1039965045 \backslash \mathrm{H},-1.4024123291,0.5101621508,0.11$ $04764476 \backslash \mathrm{H},-0.8691178302,-1.7420055957,0.2857880687 \backslash \mathrm{H}, 0.7869997865,-1$. $4474774168,0.0075270039 \backslash \mathrm{H}, 0.2938529751,1.9976220145,0.0560770585 \backslash \backslash$ Vers ion $=\mathrm{DEC}-\mathrm{AXP}-\mathrm{OSF} / 1-\mathrm{G} 03 \mathrm{RevB} .03 \backslash$ State $=1-\mathrm{A} \backslash \mathrm{HF}=-149.0958676 \backslash \mathrm{MP} 2=-149.578368$ $1 \backslash \mathrm{RMSD}=3.729 \mathrm{e}-09 \backslash \mathrm{RMSF}=4.827 \mathrm{e}-05 \backslash \mathrm{Dipole}=-0.9252965,-0.4323694,0.3964805$ $\backslash \mathrm{PG}=\mathrm{C} 01 \quad[\mathrm{X}(\mathrm{C} 1 \mathrm{H} 4 \mathrm{~N} 2)] \backslash \backslash @$

\section{$\mathrm{NH}_{3} \mathrm{CHNH}^{+}$}

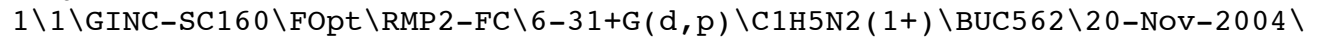
$0 \backslash \backslash \#$ MP2 $/ 6-31+G(D, P)$ OPT SYMMETRY $=$ LOOSE MAXDISK $=1342177280 \backslash \backslash \mathrm{NHCHNH} 2-\mathrm{H}+$ $\mathrm{NH} 2 \backslash \backslash 1,1 \backslash \mathrm{C}, 0.4944641295,-0.2002294227,0 . \backslash \mathrm{N},-0.2465924835,-1.216482822$ $7,0 . \backslash \mathrm{N},-0.2511049195,1.0981834785,0 . \backslash \mathrm{H}, 1.5740589992,-0.0809988657,0 . \backslash \mathrm{H}$ $, 0.2792303035,-2.0919547864,0 . \backslash \mathrm{H},-1.2547403704,0.8727173653,0 . \backslash \mathrm{H},-0.04$ $07259443,1.664854116,0.8291057834 \backslash \mathrm{H},-0.0407259443,1.664854116,-0.82910$ $57834 \backslash \backslash$ Version=DEC-AXP-OSF $/ 1-G 03$ RevB.03 $\backslash$ State $=1-A^{\prime} \backslash \mathrm{HF}=-149.4264037 \backslash \mathrm{MP} 2$ $=-149.9051792 \backslash \mathrm{RMSD}=8.745 \mathrm{e}-09 \backslash \mathrm{RMSF}=1.268 \mathrm{e}-04 \backslash \mathrm{Dipole}=0.4421325,1.5153607$ , $0 . \backslash \mathrm{PG}=\mathrm{CS}[\mathrm{SG}(\mathrm{C} 1 \mathrm{H} 3 \mathrm{~N} 2), \mathrm{X}(\mathrm{H} 2)] \backslash \backslash @$

\section{$\mathrm{NH}_{2} \mathrm{CHNH}_{2}^{+}$}

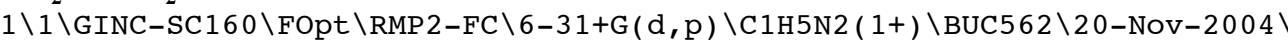
$0 \backslash \backslash \#$ MP2 $/ 6-31+G(D, P)$ OPT SYMMETRY=LOOSE MAXDISK $=1342177280 \backslash \backslash \mathrm{NHCHNH} 2-\mathrm{H}+$ $\mathrm{NH} \backslash \backslash 1,1 \backslash \mathrm{C}, 0.4314591663,0 ., 0 . \backslash \mathrm{N},-0.1766320947,-1.1659591339,0 . \backslash \mathrm{N},-0.17$ $66320947,1.1659591339,0 . \backslash \mathrm{H}, 1.5135663843,0 ., 0 . \backslash \mathrm{H}, 0.3693726098,-2.015874$ $6686,0 . \backslash \mathrm{H}, 0.3693726098,2.0158746686,0 . \backslash \mathrm{H},-1.1841086381,-1.2618455194,0$ $. \backslash \mathrm{H},-1.1841086381,1.2618455194,0 . \backslash \backslash$ Version=DEC-AXP-OSF / 1-G03RevB.03 $\backslash \mathrm{St}$ ate $=1-\mathrm{A} 1 \backslash \mathrm{HF}=-149.4826188 \backslash \mathrm{MP} 2=-149.952404 \backslash \mathrm{RMSD}=4.779 \mathrm{e}-09 \backslash \mathrm{RMSF}=1.312 \mathrm{e}-04$ $\backslash$ Dipole $=-0.0715095,0 ., 0 . \backslash \mathrm{PG}=\mathrm{C} 02 \mathrm{~V}[\mathrm{C} 2(\mathrm{C} 1 \mathrm{H} 1), \mathrm{SGV}(\mathrm{H} 4 \mathrm{~N} 2)] \backslash \backslash @$

\section{$\mathrm{NH}_{2} \mathrm{CHNH} \cdots \mathrm{HF}$ (N1)}

$1 \backslash 1 \backslash G I N C-L C 45 \backslash F O p t \backslash R M P 2-F C \backslash 6-31+G(d, p) \backslash C 1 H 5 F 1 N 2 \backslash B U C 562 \backslash 22-O c t-2004 \backslash 0 \backslash \backslash$ \# MP2 $/ 6-31+G(D, P) \quad O P T$ MAXDISK $=268435456 \backslash \backslash$ NHCHNH2 - HF $\backslash \backslash 0,1 \backslash C, 0.4417987$ $223,-0.794525836,0.6619584722 \backslash \mathrm{N}, 1.7186056851,-0.7081919697,0.661648801$ $1 \backslash \mathrm{N},-0.3461480558,0.366792857,0.6959698697 \backslash \mathrm{H},-0.1387315356,-1.71315768$ $28,0.5739308136 \backslash \mathrm{H}, 2.130891958,-1.6331602256,0.5549119326 \backslash \mathrm{H},-1.19172066$ $74,0.2683050403,1.2463123621 \backslash \mathrm{H}, 0.2019613282,1.1671817906,0.9986547094 \backslash$ $\mathrm{H},-0.965227292,0.7475175651,-0.9257212205 \backslash \mathrm{F},-1.3661299478,0.9244735909$ ,$-1.7692411251 \backslash \backslash$ Version $=x 86-$ Linux-G03RevB.03 $\backslash$ State $=1-A \backslash H F=-249.1294742$ $\backslash \mathrm{MP} 2=-249.8086959 \backslash \mathrm{RMSD}=4.201 \mathrm{e}-09 \backslash \mathrm{RMSF}=8.189 \mathrm{e}-05 \backslash \mathrm{Dipole}=-0.1599069,-0.8$ $040411,1.466591 \backslash \mathrm{PG}=\mathrm{C} 01 \quad[\mathrm{X}(\mathrm{C} 1 \mathrm{H} 5 \mathrm{~F} 1 \mathrm{~N} 2)] \backslash \backslash @$

\section{$\mathrm{NH}_{2} \mathrm{CHNH} \cdots \mathrm{HF}(\mathrm{N} 3)$}

$1 \backslash 1 \backslash G I N C-L C 52 \backslash F O p t \backslash R M P 2-F C \backslash 6-31+G(d, p) \backslash C 1 H 5 F 1 N 2 \backslash B U C 562 \backslash 22-O c t-2004 \backslash 0 \backslash \backslash$ \# MP2 $/ 6-31+\mathrm{G}(\mathrm{D}, \mathrm{P})$ OPT MAXDISK $=268435456 \backslash \backslash \mathrm{NHCHNH} 2-\mathrm{HF} \backslash \backslash 0,1 \backslash \mathrm{C},-0.398930$ $6142,-0.4865763582,0.9954259766 \backslash \mathrm{N}, 0.6354886952,-0.8921117581,0.3318315$ $484 \backslash \mathrm{N},-1.067710114,0.6424974252,0.6537092133 \backslash \mathrm{H},-0.7785140939,-0.989784$ $0021,1.8831159327 \backslash \mathrm{H}, 0.8862218241,-0.0465070919,-0.9832293396 \backslash \mathrm{F}, 0.78569$ $48381,0.61472,4 \mathrm{I} 574028554523 \backslash \mathrm{H},-1.9786159646,0.8138839949,1.040642$ $7866 \backslash \mathrm{H},-0.8316282936,1.0822561203,-0.2270576133 \backslash \mathrm{H}, 1.0504166017,-1.7256$ $133299,0.731886113 \backslash \backslash$ Version $=x 86-$ Linux-G03RevB $.03 \backslash$ State $=1-A \backslash H F=-249.139$ $9856 \backslash \mathrm{MP} 2=-249.8199218 \backslash \mathrm{RMSD}=2.059 \mathrm{e}-09 \backslash \mathrm{RMSF}=2.843 \mathrm{e}-05 \backslash \mathrm{Dipole}=-1.1274985$, $-0.6226714,1.602271 \backslash \mathrm{PG}=\mathrm{C} 01 \quad[\mathrm{X}(\mathrm{C} 1 \mathrm{H} 5 \mathrm{~F} 1 \mathrm{~N} 2)] \backslash \backslash @$ 


\section{$\mathrm{C}_{2} \mathrm{H}_{2} \mathrm{NH}(1 \mathrm{H}$-Azirine)}

$1 \backslash 1 \backslash$ GINC-LC92 2 FOpt \RMP2-FC $66-31+G(d, p) \backslash C 2 H 3 N 1 \backslash B U C 562 \backslash 05-N o v-2004 \backslash 0 \backslash \backslash \#$ MP2 $/ 6-31+G(D, P) \quad O P T$ SYMMETRY $=$ LOOSE MAXDISK $=671088640 \backslash \backslash C 2 \mathrm{H} 2 \mathrm{NH} \backslash \backslash 0,1 \backslash \mathrm{N}, 0$. $, 0.0818606688,-0.8977241143 \backslash \mathrm{C},-0.6442374945,0.0051517688,0.4797792399 \backslash$ $\mathrm{C}, 0.6442374945,0.0051517688,0.4797792399 \backslash \mathrm{H},-1.6219883347,0.1162107492$, $0.9054928741 \backslash \mathrm{H}, 1.6219883347,0.1162107492,0.9054928741 \backslash \mathrm{H}, 0 .,-0.86726740$ $58,-1.2842678261 \backslash \backslash$ Version $=x 86-$ Linux-G03RevB.03 $\backslash$ State $=1-A^{\prime} \backslash \mathrm{HF}=-131.7862$ $366 \backslash M P 2=-132.2294911 \backslash \mathrm{RMSD}=3.330 \mathrm{e}-09 \backslash \mathrm{RMSF}=6.734 \mathrm{e}-05 \backslash \mathrm{Dipole}=0 .,-0.528426$ $7,0.6740716 \backslash \mathrm{PG}=\mathrm{CS} \quad[\mathrm{SG}(\mathrm{H} 1 \mathrm{~N} 1), \mathrm{X}(\mathrm{C} 2 \mathrm{H} 2)] \backslash \backslash @$

\section{$\mathrm{C}_{2} \mathrm{H}_{2} \mathrm{NH}_{2}^{+}$}

$1 \backslash 1 \backslash$ GINC-LC52 \FOpt \RMP2-FC $\backslash 6-31+\mathrm{G}(\mathrm{d}, \mathrm{p}) \backslash \mathrm{C} 2 \mathrm{H} 4 \mathrm{~N} 1(1+) \backslash \mathrm{BUC} 562 \backslash 05-\mathrm{Nov}-2004 \backslash 0$ $\backslash \backslash \#$ MP2/6-31+G(D,P) OPT SYMMETRY=LOOSE MAXDISK $=671088640 \backslash \backslash \mathrm{C} 2 \mathrm{H} 2 \mathrm{NH} 2+\backslash \backslash 1$, $1 \backslash \mathrm{N}, 0 ., 0 \ldots,-0.8077244506 \backslash \mathrm{C},-0.6434634587,0 ., 0.5462900738 \backslash \mathrm{C}, 0.6434634587$ $, 0 ., 0.5462900738 \backslash \mathrm{H},-1.6570823274,0 ., 0.9057423982 \backslash \mathrm{H}, 1.6570823274,0 ., 0.9$ $057423982 \backslash \mathrm{H}, 0 .,-0.8589982977,-1.3564472639 \backslash \mathrm{H}, 0 ., 0.8589982977,-1.356447$ $2639 \backslash \backslash$ Version $=x 86-$ Linux $-G 03 R e v B .03 \backslash$ State $=1-A 1 \backslash H F=-132.167664 \backslash \mathrm{MP} 2=-132$. $5991592 \backslash \mathrm{RMSD}=6.771 \mathrm{e}-09 \backslash \mathrm{RMSF}=1.423 \mathrm{e}-04 \backslash \mathrm{Dipole}=0 ., 0 .,-0.4846179 \backslash \mathrm{PG}=\mathrm{C} 02 \mathrm{~V}$ $\left[\mathrm{C} 2(\mathrm{~N} 1), \mathrm{SGV}(\mathrm{C} 2 \mathrm{H} 2), \mathrm{SGV}^{\prime}(\mathrm{H} 2)\right] \backslash \backslash @$

\section{$\mathrm{C}_{2} \mathrm{H}_{3} \mathrm{NH}^{+}$}

$1 \backslash 1 \backslash$ GINC-LC92 FOpt $\backslash$ RMP2-FC $\backslash 6-31+G(d, p) \backslash C 2 H 4 N 1(1+) \backslash B U C 562 \backslash 05-N o v-2004 \backslash 0$ $\backslash \backslash \#$ MP $2 / 6-31+G(D, P)$ OPT SYMMETRY=LOOSE MAXDISK $=671088640 \backslash \backslash C 2 \mathrm{H} 2 \mathrm{NH} 2+\backslash \backslash 1$, $1 \backslash \mathrm{C},-0.8242144238,-0.3466103702,0 . \backslash \mathrm{N}, 0.6855827842,-0.3390164707,0 . \backslash \mathrm{C}, 0$ $.1277157854,0.7903569494,0 . \backslash \mathrm{H}, 1.5720612029,-0.8449107676,0 . \backslash \mathrm{H}, 0.378328$ $2029,1.841965508,0 . \backslash \mathrm{H},-1.2852385322,-0.6432094606,-0.9316395248 \backslash \mathrm{H},-1.2$ $852385322,-0.6432094606,0.9316395248 \backslash \backslash$ Version $=x 86-L$ inux-G03RevB.03 \Sta te $=1-A^{\prime} \backslash \mathrm{HF}=-132.1775716 \backslash \mathrm{MP} 2=-132.6047779 \backslash \mathrm{RMSD}=3.861 \mathrm{e}-09 \backslash \mathrm{RMSF}=1.358 \mathrm{e}-04$ $\backslash$ Dipole $=0.4550341,0.1255662,0 . \backslash \mathrm{PG}=\mathrm{CS}[\mathrm{SG}(\mathrm{C} 2 \mathrm{H} 2 \mathrm{~N} 1), \mathrm{X}(\mathrm{H} 2)] \backslash \backslash @$

\section{$\mathrm{C}_{2} \mathrm{H}_{2} \mathrm{NH} \cdots \cdot \mathrm{HF}(\mathrm{N} 1)$}

$1 \backslash 1 \backslash G I N C-S C 79 \backslash F O p t \backslash R M P 2-F C \backslash 6-31+G(d, p) \backslash C 2 H 4 F 1 N 1 \backslash B U C 562 \backslash 05-N o v-2004 \backslash 0 \backslash \backslash$ \# MP2 /6-31+G(D,P) OPT SYMMETRY=LOOSE MAXDISK $=1342177280 \backslash \backslash \mathrm{C} 2 \mathrm{H} 2 \mathrm{NH}-\mathrm{HF} \backslash \backslash$ $0,1 \backslash \mathrm{N}, 0 .,-0.6222815678,0.2520902 \backslash \mathrm{C},-0.6440691534,0.2678165104,1.294636$ $6495 \backslash \mathrm{C}, 0.6440691534,0.2678165104,1.2946366495 \backslash \mathrm{H},-1.6311960231,0.607898$ $674,1.5376504587 \backslash \mathrm{H}, 1.6311960231,0.607898674,1.5376504587 \backslash \mathrm{H}, 0 .,-1.59186$ $33571,0.5750126459 \backslash \mathrm{H}, 0 .,-0.1557091615,-1.2734854499 \backslash \mathrm{F}, 0 ., 0.1859942245$, $-2.1863443675 \backslash \backslash$ Version=DEC-AXP-OSF / $1-$ G03RevB.03 $\backslash$ State $=1-A^{\prime} \backslash \mathrm{HF}=-231.830$ $5541 \backslash M P 2=-232.4714092 \backslash \mathrm{RMSD}=5.405 \mathrm{e}-09 \backslash \mathrm{RMSF}=1.324 \mathrm{e}-04 \backslash \mathrm{Dipole}=0 .,-0.28569$ $07,2.1905773 \backslash \mathrm{PG}=\mathrm{CS}[\mathrm{SG}(\mathrm{H} 2 \mathrm{~F} 1 \mathrm{~N} 1), \mathrm{X}(\mathrm{C} 2 \mathrm{H} 2)] \backslash \backslash @$

\section{$\mathrm{C}_{2} \mathrm{H}_{2} \mathrm{NH} \cdots \cdot \mathrm{HF}(\mathrm{C}=\mathrm{C})$}

$1 \backslash 1 \backslash$ GINC-SC105\FOpt $\backslash$ RMP $2-F C \backslash 6-31+G(d, p) \backslash C 2 H 4 F 1 N 1 \backslash B U C 562 \backslash 16-F e b-2005 \backslash 0 \backslash$ $\backslash \#$ MP2 $/ 6-31+G(D, P)$ OPT SYMMETRY=LOOSE MAXDISK $=268435456 \backslash \backslash C 2 \mathrm{H} 2 \mathrm{NH}-\mathrm{HF} \backslash \backslash$ $0,1 \backslash \mathrm{C}, 0.6465109857,0.7058430184,-0.4245569757 \backslash \mathrm{C},-0.6465109857,0.705843$ $0184,-0.4245569757 \backslash \mathrm{N}, 0 ., 1.6862556116,0.535368594 \backslash \mathrm{H}, 1.620619783,0.51534$ $84412,-0.8333303947 \backslash \mathrm{H},-1.620619783,0.5153484412,-0.8333303947 \backslash \mathrm{H}, 0 ., 1.2$ $571388369,1.4655731928 \backslash \mathrm{H}, 0 .,-1.4290714701,0.0027153659 \backslash \mathrm{F}, 0 .,-2.3480744$ $168,0.1717195313 \backslash \backslash$ Version=DEC-AXP-OSF $/ 1-G 03$ RevB.03 $\backslash$ State $=1-A^{\prime} \backslash \mathrm{HF}=-231$. $8124033 \backslash M P 2=-232.4511188 \backslash \mathrm{RMSD}=8.929 \mathrm{e}-09 \backslash \mathrm{RMSF}=8.560 \mathrm{e}-06 \backslash \mathrm{Dipole}=0,0.240$ $6345,-0.3029516 \backslash \mathrm{PG}=\mathrm{CS}[\mathrm{SG}(\mathrm{H} 2 \mathrm{~F} 1 \mathrm{~N} 1), \mathrm{X}(\mathrm{C} 2 \mathrm{H} 2)] \backslash \backslash @$

\section{$\mathrm{NH}_{2} \mathrm{CNCH}_{2}$ (Vinylamine)}

$1 \backslash 1 \backslash G I N C-S C 6 \backslash F O p t \backslash R M P 2-F C \backslash 6-31+G(d, p) \backslash C 2 H 5 N 1 \backslash B U C 562 \backslash 17-O c t-2004 \backslash 0 \backslash \backslash \# M$ $\mathrm{P} 2 / 6-31+\mathrm{G}(\mathrm{D}, \mathrm{P}) \quad \mathrm{OPT}$ MAXDISK $=134217728 \backslash \backslash \mathrm{CH} 2 \mathrm{CHNH} 2 \backslash \backslash 0,1 \backslash \mathrm{C},-0.0265662002,0$. $0880437644,0.4328072704 \backslash \mathrm{C}, 0.5107625987,1.1422587932,-0.2052880791 \backslash \mathrm{N},-0$ $.509175704,-1.0765444356,-0.1728035802 \backslash \mathrm{H},-0.1894846433,0.1207497832,1$. $5036628283 \backslash \mathrm{H}, 0.6737807334,1.1405668206,-1.274931589 \backslash \mathrm{H}, 0.7747252496,2.0$ $300825813,0.3468178305 \backslash \mathrm{H},-0.4201253798,-1.9121977475,0.3856311817 \backslash \mathrm{H},-0$ $.1798444232,-1.225205734,-1.1166703381 \backslash \backslash$ Version=DEC-AXP-OSF / 1-G03RevB . $03 \backslash$ State $=1-A \backslash H F=-133.0813035 \backslash M P 2=-133.5343736 \backslash \mathrm{RMSD}=6.267 \mathrm{e}-09 \backslash \mathrm{RMSF}=1.76$ $5 e-05 \backslash$ Dipole $=0.2529651,-0.5292163,0.0482915 \backslash P G=C 01 \quad[X(C 2 H 5 N 1)] \backslash \backslash @$ 


\section{$\mathrm{NH}_{3} \mathrm{CHCH}_{2}^{+}$}

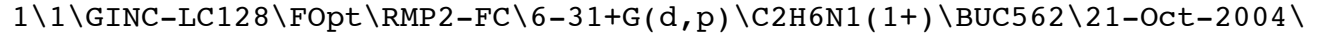
$0 \backslash \backslash \#$ MP2 $/ 6-31+G(D, P) \quad O P T$ MAXDISK $=268435456 \backslash \backslash \mathrm{CH} 2 \mathrm{CHNH} 3+\backslash \backslash 1,1 \backslash \mathrm{C},-0.514580$ $7551,0 .,-0.1210951944 \backslash \mathrm{C},-0.5121965317,0 ., 1.2078209389 \backslash \mathrm{N}, 0.7635273481,0$ $.,-0.8724552715 \backslash \mathrm{H},-1.3837636671,0 .,-0.760819131 \backslash \mathrm{H}, 0.3934261284,0 ., 1.80$ $02404496 \backslash \mathrm{H},-1.4561380609,0 ., 1.7314632411 \backslash \mathrm{H}, 0.8481900865,-0.825945238,-$ $1.4742362428 \backslash \mathrm{H}, 1.5660677107,0 .,-0.2355796411 \backslash \mathrm{H}, 0.8481900865,0.82594523$ $8,-1.4742362428 \backslash \backslash$ Version $=x 86-$ Linux-G03RevB. 03 $\backslash$ State $=1-A^{\prime} \backslash \mathrm{HF}=-133.42888$ $02 \backslash M P 2=-133.8770898 \backslash \mathrm{RMSD}=6.255 \mathrm{e}-09 \backslash \mathrm{RMSF}=7.433 \mathrm{e}-05 \backslash \mathrm{Dipole}=1.0435376,0$. $-1.0549248 \backslash \mathrm{PG}=\mathrm{CS} \quad[\mathrm{SG}(\mathrm{C} 2 \mathrm{H} 4 \mathrm{~N} 1), \mathrm{X}(\mathrm{H} 2)] \backslash \backslash @$

\section{$\mathrm{NH}_{2} \mathrm{CHCH}_{3}^{+}$}

$1 \backslash 1 \backslash G I N C-L C 133 \backslash F O p t \backslash R M P 2-F C \backslash 6-31+G(d, p) \backslash C 2 H 6 N 1(1+) \backslash B U C 562 \backslash 21-O C t-2004 \backslash$ $0 \backslash \backslash \#$ MP2 $/ 6-31+G(D, P)$ OPT SYMMETRY=LOOSE MAXDISK $=268435456 \backslash \backslash \mathrm{CH} 3 \mathrm{CHNH} 2+\backslash \backslash$ $1,1 \backslash \mathrm{C},-0.3822393895,-0.0000002319,-0.2696206133 \backslash \mathrm{C},-0.3729513495,-0.000$ $0005816,1.2027177088 \backslash \mathrm{N}, 0.6877527279,0.0000007751,-0.9912084612 \backslash \mathrm{H},-1.32$ $31355817,-0.0000008326,-0.8097066196 \backslash \mathrm{H},-0.9237058581,0.875289719,1.552$ $8875983 \backslash \mathrm{H},-0.9237044968,-0.8752919085,1.5528871739 \backslash \mathrm{H}, 0.6438765721,0.00$ $00009871,-2.0063131406 \backslash \mathrm{H}, 1.6124261863,0.0000013916,-0.568091221 \backslash \mathrm{H}, 0.63$ $11185168,0.0000000985,1.6182128648 \backslash \backslash$ Version=x86-Linux-G03RevB.03\State $=1-\mathrm{A}^{\prime} \backslash \mathrm{HF}=-133.4577149 \backslash \mathrm{MP} 2=-133.8998898 \backslash \mathrm{RMSD}=4.180 \mathrm{e}-09 \backslash \mathrm{RMSF}=8.843 \mathrm{e}-05 \backslash \mathrm{D}$ ipole $=0.3003603,0.0000004,-0.7632376 \backslash \mathrm{PG}=\mathrm{CS} \quad[\mathrm{SG}(\mathrm{C} 2 \mathrm{H} 4 \mathrm{~N} 1), \mathrm{X}(\mathrm{H} 2)] \backslash \backslash @$

\section{$\mathrm{NH}_{2} \mathrm{CHCH}_{2} \cdots \cdot \mathrm{HF}$ (N1)}

$1 \backslash 1 \backslash \mathrm{GINC}-\mathrm{SC} 90 \backslash \mathrm{FOpt} \backslash \mathrm{RMP} 2-\mathrm{FC} \backslash 6-31+\mathrm{G}(\mathrm{d}, \mathrm{p}) \backslash \mathrm{C} 2 \mathrm{H} 6 \mathrm{~F} 1 \mathrm{~N} 1 \backslash \mathrm{BUC} 562 \backslash 13-$ Oct $-2004 \backslash 0 \backslash \backslash$ \# MP2 /6-31+G(D,P) OPT MAXDISK $=268435456 \backslash \backslash \mathrm{CH} 2 \mathrm{CHNH} 2-\mathrm{HF} \backslash \backslash 0,1 \backslash \mathrm{C}, 0.299317$ $2888,-0.8504525906,0.6691891955 \backslash \mathrm{C}, 1.6305433072,-0.914369693,0.79781249$ $36 \backslash \mathrm{N},-0.4512589142,0.3571514471,0.6346770703 \backslash \mathrm{H},-0.2954522729,-1.738222$ $1689,0.4964176749 \backslash \mathrm{H}, 2.2336736536,-0.0295008466,0.9522802399 \backslash \mathrm{H}, 2.134262$ $5715,-1.8655826166,0.7304252601 \backslash \mathrm{H},-1.3218433577,0.2787013897,1.1502570$ Q01. $877 \mathrm{H} 478504,1.1377460746,1.0119095061 \backslash \mathrm{H},-0.8867315091,0.7915211$ $296,-0.9650459155 \backslash F,-1.1513342346,1.0571345122,-1.8467770438 \backslash \backslash$ Version= DEC-AXP-OSF $/ 1-G 03$ RevB.03 $\backslash$ State $=1-\mathrm{A} \backslash \mathrm{HF}=-233.1179167 \backslash \mathrm{MP} 2=-233.7685466 \backslash \mathrm{RM}$ $\mathrm{SD}=8.542 \mathrm{e}-09 \backslash \mathrm{RMSF}=2.468 \mathrm{e}-05 \backslash \mathrm{Dipole}=0.2799168,-0.3471366,1.6249359 \backslash \mathrm{PG}=\mathrm{C}$ $01[\mathrm{X}(\mathrm{C} 2 \mathrm{H} 6 \mathrm{~F} 1 \mathrm{~N} 1)] \backslash \backslash \varrho$

\section{$\mathrm{NH}_{2} \mathrm{CHCH}_{2} \cdots \cdot \mathrm{HF}(\mathrm{C} 3)$}

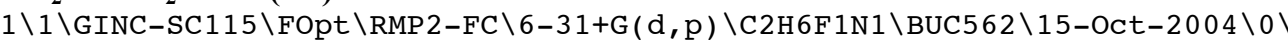
$\backslash \#$ MP2 $/ 6-31+G(D, P)$ OPT MAXDISK $=268435456 \backslash \backslash \mathrm{CH} 2 \mathrm{CHNH} 2-\mathrm{HF} \backslash \backslash 0,1 \backslash \mathrm{C},-0.8961$ $560088,0.2305419658,0.415122 \backslash \mathrm{C},-0.3368570491,1.2858569871,-0.217675 \backslash \mathrm{N}$, $-1.3600279646,-0.926625052,-0.185271 \backslash \mathrm{H},-1.05456101,0.2627049597,1.4869$ $55 \backslash \mathrm{H},-0.2078660497,1.3018639921,-1.292437 \backslash \mathrm{H},-0.1222660836,2.1879669953$ $, 0.333693 \backslash \mathrm{H}, 1.3780719947,0.1390240526,0.069809 \backslash \mathrm{F}, 2.1451710155,-0.40619$ $69181,0.02995 \backslash \mathrm{H},-1.3264579327,-1.7610690507,0.37937 \backslash \mathrm{H},-1.0551809585,-1$ $.0867460403,-1.134723 \backslash \backslash$ Version=DEC-AXP-OSF $/ 1-G 03$ RevB.03 $\backslash$ State $=1-\mathrm{A} \backslash \mathrm{HF}=-$ $233.1118872 \backslash M P 2=-233.7614412 \backslash \mathrm{RMSD}=5.723 \mathrm{e}-09 \backslash \mathrm{RMSF}=3.715 \mathrm{e}-05 \backslash \mathrm{Dipole}=-0.8$ $406231,-0.114559,0.0733844 \backslash \mathrm{PG}=\mathrm{C} 01 \quad[\mathrm{X}(\mathrm{C} 2 \mathrm{H} 6 \mathrm{~F} 1 \mathrm{~N} 1)] \backslash \backslash @$

\section{$\mathrm{C}_{3} \mathrm{H}_{3} \mathrm{~N}$ (Azete)}

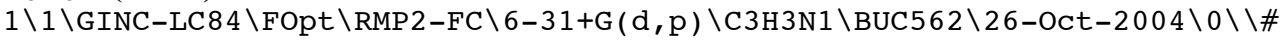
MP2 $/ 6-31+G(D, P)$ OPT MAXDISK $=268435456 \backslash \backslash \mathrm{C} 3 \mathrm{H} 3 \mathrm{~N} \backslash \backslash 0,1 \backslash \mathrm{N},-0.7260109878,-0.8$ $159714947,0 . \backslash C, 0.5722116659,-0.7769675586,0 . \backslash C, 0.7024064701,0.75765541$ $95,0 . \backslash \mathrm{C},-0.6392141117,0.7497447306,0 . \backslash \mathrm{H}, 1.2581207858,-1.6164381027,0 . \backslash$ $\mathrm{H}, 1.480433088,1.5058453399,0 . \backslash \mathrm{H},-1.4689011048,1.4397976766,0 . \backslash \backslash$ Version $=x 86-$ Linux-G03RevB.03 $\backslash$ State $=1-A^{\prime} \backslash \mathrm{HF}=-169.6531649 \backslash \mathrm{MP} 2=-170.2177811 \backslash \mathrm{RMSD}$ $=4.418 \mathrm{e}-09 \backslash \mathrm{RMSF}=1.606 \mathrm{e}-04 \backslash \mathrm{Dipole}=0.7414194,0.624979,0 . \backslash \mathrm{PG}=\mathrm{CS} \quad[\mathrm{SG}(\mathrm{C} 3 \mathrm{H} 3 \mathrm{~N}$ 1) $] \backslash \backslash @$

\section{$\mathrm{C}_{3} \mathrm{H}_{3} \mathrm{NH}^{+}$}

$1 \backslash 1 \backslash$ GINC-SC160\FOpt $\backslash$ RMP $2-F C \backslash 6-31+G(d, p) \backslash C 3 H 4 N 1(1+) \backslash B U C 562 \backslash 20-N o v-2004 \backslash$ $0 \backslash \backslash \#$ MP2 $/ 6-31+G(D, P) \quad O P T$ SYMMETRY=LOOSE MAXDISK $=1342177280 \backslash \backslash C 3 \mathrm{H} 3 \mathrm{NH}+\backslash \backslash 1$ $, 1 \backslash \mathrm{N},-0.962788242,0.2185619701,0 . \backslash \mathrm{C}, 0.0847321858,0.9705617253,0 . \backslash \mathrm{C}, 1.0$ $49357454,-0.2077572252,0 . \backslash \mathrm{C},-0.008231662,-1.0319027574,0 . \backslash \mathrm{H}, 0.13873316$ $8,2.052850844,0 . \backslash \mathrm{H}, 2.1194740612,-0.3191125363,0 . \backslash \mathrm{H},-0.3131901774,-2.06$ $61916153,0 . \backslash \mathrm{H},-1.9606472251,0.4171090599,0 . \backslash \backslash$ Version=DEC-AXP-OSF $/ 1-\mathrm{G} 03$ RevB.03\State $=1-A^{\prime} \backslash H F=-170.0173472 \backslash M P 2=-170.5629732 \backslash$ RMSD $=5.208 \mathrm{e}-09 \backslash$ RMS $\mathrm{F}=8.041 \mathrm{e}-05 \backslash \mathrm{Dipole}=-0.4839423,0.4217391,0 . \backslash \mathrm{PG}=\mathrm{CS}[\mathrm{SG}(\mathrm{C} 3 \mathrm{H} 4 \mathrm{~N} 1)] \backslash \backslash @$ 


\section{$\mathrm{C}_{3} \mathrm{H}_{4} \mathbf{N}^{+}(\mathrm{C} 2)$}

$1 \backslash 1 \backslash$ GINC-LC86\FOpt \RMP2-FC $\backslash 6-31+G(\mathrm{~d}, \mathrm{p}) \backslash \mathrm{C} 3 \mathrm{H} 4 \mathrm{~N} 1(1+) \backslash \mathrm{BUC} 562 \backslash 26-$ Oct $-2004 \backslash 0$ $\backslash \backslash \#$ MP2 $/ 6-31+G(D, P) \quad O P T$ MAXDISK $=268435456 \backslash \backslash C 3 \mathrm{H} 4 \mathrm{~N}+\backslash \backslash 1,1 \backslash \mathrm{N},-0.7402572757$ $, 0.2573575506,-0.4754115367 \backslash \mathrm{C},-0.0036552261,1.0505445795,0.2723864924 \backslash$ $C, 0.8736442729,-0.0662542433,0.1564621598 \backslash C,-0.165900786,-1.0920639506$ $, 0.0713585371 \backslash \mathrm{H},-0.035517463,2.1132611506,0.4640009464 \backslash \mathrm{H}, 1.7740544971$, $-0.0466533098,-0.4530582154 \backslash \mathrm{H},-0.0705473636,-1.8879710324,-0.657813170$ $2 \backslash \mathrm{H},-0.7107183056,-1.3334979761,0.9735080596 \backslash \backslash$ Version=x86-Linux-G03Rev B. $03 \backslash$ State $=1-A \backslash H F=-169.9997541 \backslash M P 2=-170.5573031 \backslash \mathrm{RMSD}=3.501 \mathrm{e}-09 \backslash \mathrm{RMSF}=1$. $210 \mathrm{e}-04 \backslash \mathrm{Dipole}=0.6078653,0.1598968,0.4002491 \backslash \mathrm{PG}=\mathrm{C} 01 \quad[\mathrm{X}(\mathrm{C} 3 \mathrm{H} 4 \mathrm{~N} 1)] \backslash \backslash \mathrm{C}$

\section{$\mathrm{C}_{3} \mathbf{H}_{4} \mathbf{N}^{+}(\mathrm{C} 3)$}

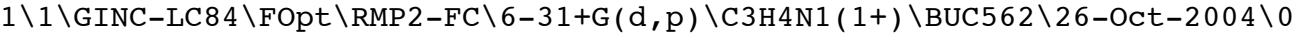
$\backslash \backslash \#$ MP2 $/ 6-31+G(D, P)$ OPT SYMMETRY=LOOSE MAXDISK $=268435456 \backslash \backslash C 3 \mathrm{H} 4 \mathrm{~N}+\backslash \backslash 1,1 \backslash$ $\mathrm{N},-1.071018,0.0439863165,0.1925046106 \backslash \mathrm{C},-0.069,0.7941743145,-0.3289708$ $925 \backslash C, 1.139456,0.0294761292,0.1290012716 \backslash C,-0.069,-0.8582383288,0.0485$ $969242 \backslash \mathrm{H},-0.217,1.5098737182,-1.1338177125 \backslash \mathrm{H}, 1.98751,-0.1214458577,-0$. $5315036448 \backslash \mathrm{H},-0.217,-1.8524691767,-0.3655396004 \backslash \mathrm{H}, 1.395766,0.264720481$ $3,1.1585401374 \backslash \backslash$ Version $=x 86-$ Linux $^{2}$ G03RevB.03 $\backslash$ State $=1-\mathrm{A}^{\prime} \backslash \mathrm{HF}=-170.021145$ $4 \backslash \mathrm{MP} 2=-170.5665926 \backslash \mathrm{RMSD}=2.968 \mathrm{e}-09 \backslash \mathrm{RMSF}=7.568 \mathrm{e}-05 \backslash \mathrm{Dipole}=0,,-0.4602032$, $0.8770513 \backslash \mathrm{PG}=\mathrm{CS} \quad[\mathrm{SG}(\mathrm{C} 1 \mathrm{H} 2 \mathrm{~N} 1), \mathrm{X}(\mathrm{C} 2 \mathrm{H} 2)] \backslash \backslash @$

\section{$\mathrm{C}_{3} \mathrm{H}_{3} \mathrm{~N} \cdots \cdot \mathrm{HF}(\mathrm{N} 1)$}

$1 \backslash 1 \backslash G I N C-S C 160 \backslash F O p t \backslash R M P 2-F C \backslash 6-31+G(d, p) \backslash C 3 H 4 F 1 N 1 \backslash B U C 562 \backslash 20-N o v-2004 \backslash 0 \backslash$ $\backslash \#$ MP2 $/ 6-31+G(D, P)$ OPT SYMMETRY $=$ LOOSE MAXDISK $=1342177280 \backslash \backslash \mathrm{C} 3 \mathrm{H} 3 \mathrm{~N}-\mathrm{HF} \backslash \backslash$ $0,1 \backslash \mathrm{N},-0.1101473464,0.0650298937,0 . \backslash \mathrm{C}, 0.964177742,0.7885995328,0 . \backslash \mathrm{C}, 1$. $9494919421,-0.3933256282,0 . \backslash \mathrm{C}, 0.8541436542,-1.1670197736,0 . \backslash \mathrm{H}, 1.033259$ $7286,1.8697859002,0 . \backslash \mathrm{H}, 3.0164272716,-0.5488204839,0 . \backslash \mathrm{H}, 0.5690423145,-2$ $.2071331776,0 . \backslash \mathrm{H},-1.7995974826,0.3534414633,0 . \backslash \mathrm{F},-2.7394422708,0.52311$ $02507,0 . \backslash \backslash$ Version=DEC-AXP-OSF $/ 1-G 03 R e v B .03 \backslash$ State $=1-A^{\prime} \backslash \mathrm{HF}=-269.6941135 \backslash$ MP $2=-270.4529966 \backslash \mathrm{RMSD}=9.869 \mathrm{e}-09 \backslash \mathrm{RMSF}=8.869 \mathrm{e}-05 \backslash \mathrm{Dipole}=2.3034626,-0.296$ $3323,0 . \backslash \mathrm{PG}=\mathrm{CS}[\mathrm{SG}(\mathrm{C} 3 \mathrm{H} 4 \mathrm{~F} 1 \mathrm{~N} 1)] \backslash \backslash @$

\section{$\mathrm{C}_{3} \mathrm{H}_{3} \mathrm{~N} \cdots \cdot \mathrm{HF}(\mathrm{C3})$}

$1 \backslash 1 \backslash$ GINC-LC85 $\backslash$ FOpt $\backslash R M P 2-F C \backslash 6-31+G(d, p) \backslash C 3 H 4 F 1 N 1 \backslash B U C 562 \backslash 27-O C t-2004 \backslash 0 \backslash \backslash$ \# MP2 $/ 6-31+G(D, P)$ OPT MAXDISK $=268435456 \backslash \backslash C 3 \mathrm{H} 3 \mathrm{~N}-\mathrm{HF} \backslash \backslash 0,1 \backslash \mathrm{N},-0.06378846$ $87,-1.6530234165,0 . \backslash \mathrm{C},-0.6802131878,-0.7001749425,0.797338795 \backslash \mathrm{C},-0.707$ $6334499,0.5058634438,0 . \backslash \mathrm{C},-0.6802131878,-0.7001749425,-0.797338795 \backslash \mathrm{H},-$ $1.3434833592,-0.9803212665,1.609196452 \backslash \mathrm{H},-1.5410180857,1.2034112465,0$. $\backslash \mathrm{H},-1.3434833592,-0.9803212665,-1.609196452 \backslash \mathrm{H}, 0.9512665021,1.373754346$ $8,0 . \backslash F, 1.7923996151,1.8135066114,0 . \backslash$ Version=x86-Linux-G03RevB.03 Stat $\mathrm{e}=1-\mathrm{A}^{\prime} \backslash \mathrm{HF}=-269.6635803 \backslash \mathrm{MP} 2=-270.4357726 \backslash \mathrm{RMSD}=4.679 \mathrm{e}-09 \backslash \mathrm{RMSF}=5.871 \mathrm{e}-05 \backslash$ Dipole $=-2.0825709,-0.4418262,0 . \backslash \mathrm{PG}=\mathrm{CS}[\mathrm{SG}(\mathrm{C} 1 \mathrm{H} 2 \mathrm{~F} 1 \mathrm{~N} 1), \mathrm{X}(\mathrm{C} 2 \mathrm{H} 2)] \backslash \backslash @$

\section{$\mathrm{C}_{4} \mathrm{H}_{4} \mathrm{NH}$ (Pyrrole)}

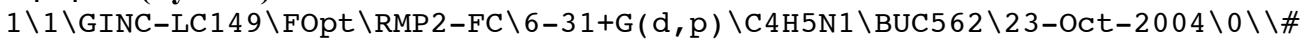
MP2 $/ 6-31+G(D, P)$ OPT SYMMETRY $=$ LOOSE MAXDISK $=268435456 \backslash \backslash \mathrm{C} 4 \mathrm{H} 4 \mathrm{NH} \backslash \backslash 0,1 \backslash \mathrm{N}, 1$ $.121331,0 ., 0 . \backslash \mathrm{C}, 0.33473,0 ., 1.12726 \backslash \mathrm{C},-0.98664,0 ., 0.7102 \backslash \mathrm{C}, 0.33473,0 \ldots,-$ $1.12726 \backslash \mathrm{C},-0.98664,0 .,-0.7102 \backslash \mathrm{H}, 2.128493,0.0 . \backslash \mathrm{H}, 0.7701,0 .,-2.11235 \backslash \mathrm{H}$, $-1.84765,0 .,-1.3586 \backslash \mathrm{H},-1.84765,0 ., 1.3586 \backslash \mathrm{H}, 0.7701,0 ., 2.11235 \backslash \backslash$ Version $=$ $\mathrm{x} 86-\mathrm{Linux}-\mathrm{G} 03$ RevB.03 $\backslash$ State $=1-\mathrm{A} 1 \backslash \mathrm{HF}=-208.8267321 \backslash \mathrm{MP} 2=-209.5376839 \backslash \mathrm{RMSD}=$ $3.238 \mathrm{e}-09 \backslash \mathrm{RMSF}=3.698 \mathrm{e}-05 \backslash \mathrm{Dipole}=0 ., 0.7793185,0 . \backslash \mathrm{PG}=\mathrm{C} 02 \mathrm{~V}[\mathrm{C} 2(\mathrm{~N} 1 \mathrm{H} 1), \mathrm{SGV}($ $\mathrm{C} 4 \mathrm{H} 4)] \backslash \backslash \mathrm{a}$

\section{$\mathrm{C}_{4} \mathrm{H}_{4} \mathrm{NH}_{2}{ }^{+}$}

$1 \backslash 1 \backslash$ GINC-LC45 $\backslash \mathrm{FOpt} \backslash \mathrm{RMP} 2-\mathrm{FC} \backslash 6-31+\mathrm{G}(\mathrm{d}, \mathrm{p}) \backslash \mathrm{C} 4 \mathrm{H} 6 \mathrm{~N} 1(1+) \backslash \mathrm{BUC} 562 \backslash 23-$ Oct $-2004 \backslash 0$

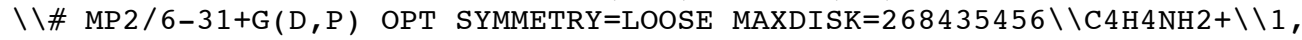
$1 \backslash \mathrm{N},-1.145374,0 ., 0 . \backslash \mathrm{C},-0.2533,0 ., 1.17965 \backslash \mathrm{C}, 1.015,0.0 .73175 \backslash \mathrm{C},-0.2533$, $0 .,-1.17965 \backslash \mathrm{C}, 1.015,0 .,-0.73175 \backslash \mathrm{H},-1.758826,0.826828,0 . \backslash \mathrm{H},-0.694782,0$. $, 2.163 \backslash \mathrm{H}, 1.8918,0 ., 1.36 \backslash \mathrm{H}, 1.8918,0 .,-1.36 \backslash \mathrm{H},-0.694782,0 .,-2.163 \backslash \mathrm{H},-1.7$ $58826,-0.826828,0 . \backslash \backslash$ Version $=x 86-$ Linux-G03RevB.03 $\backslash$ State $=1-A 1 \backslash H F=-209.15$ $04239 \backslash M P 2=-209.8471295 \backslash \mathrm{RMSD}=7.282 \mathrm{e}-09 \backslash \mathrm{RMSF}=9.786 \mathrm{e}-05 \backslash \mathrm{Dipole}=0 ., 1.21642$ $21,0 . \backslash \mathrm{PG}=\mathrm{C} 02 \mathrm{~V} \quad\left[\mathrm{C} 2(\mathrm{~N} 1), \mathrm{SGV}(\mathrm{C} 4 \mathrm{H} 4), \mathrm{SGV}^{\prime}(\mathrm{H} 2)\right] \backslash \backslash @$ 


\section{$\mathrm{C}_{4} \mathrm{H}_{5} \mathrm{NH}^{+}(\mathrm{C} 2)$}

$1 \backslash 1 \backslash \mathrm{GINC}-\mathrm{LC} 117 \backslash \mathrm{FOpt} \backslash \mathrm{RMP} 2-\mathrm{FC} \backslash 6-31+\mathrm{G}(\mathrm{d}, \mathrm{p}) \backslash \mathrm{C} 4 \mathrm{H} 6 \mathrm{~N} 1(1+) \backslash \mathrm{BUC} 562 \backslash 22-\mathrm{OCt}-2004 \backslash$

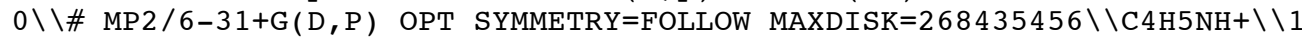
$, 1 \backslash \mathrm{N},-0.7488024846,-0.8538096752,0 . \backslash \mathrm{C}, 0.7066046478,-0.9699386006,0 . \backslash \mathrm{C}$, $1.1334472173,0.4541274559,0 . \backslash C, 0.0369048564,1.2592756657,0 . \backslash C,-1.11803$ $22593,0.40213035,0 . \backslash \mathrm{H}, 1.0339447186,-1.5172226878,0.8886935473 \backslash \mathrm{H}, 0.0026$ $36415,2.3365675528,0 . \backslash \mathrm{H},-2.1628105785,0.6820684655,0 . \backslash \mathrm{H},-1.3883226251$, $-1.6422430756,0 . \backslash \mathrm{H}, 2.1686779703,0.7611509327,0 . \backslash \mathrm{H}, 1.0339447186,-1.5172$ $226878,-0.8886935473 \backslash \backslash$ Version $=x 86-$ Linux-G03RevB.03 $\backslash$ State $=1-A^{\prime} \backslash \mathrm{HF}=-209$. $1867471 \backslash M P 2=-209.877514 \backslash \mathrm{RMSD}=5.816 \mathrm{e}-09 \backslash \mathrm{RMSF}=4.265 \mathrm{e}-05 \backslash \mathrm{Dipole}=-0.465702$ $9,-0.5500876,0 . \backslash \mathrm{PG}=\mathrm{CS}[\mathrm{SG}(\mathrm{C} 4 \mathrm{H} 4 \mathrm{~N} 1), \mathrm{X}(\mathrm{H} 2)] \backslash \backslash @$

\section{$\mathrm{C}_{4} \mathrm{H}_{5} \mathrm{NH}^{+}(\mathrm{C3})$}

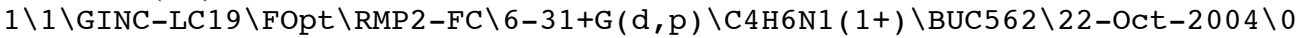
$\backslash \backslash \#$ MP2 $/ 6-31+G(D, P) \quad O P T$ MAXDISK $=268435456 \backslash \backslash C 4 \mathrm{H} 5 \mathrm{NH}+\backslash \backslash 1,1 \backslash \mathrm{N},-0.961655636$ $1,0 .,-0.623802329 \backslash \mathrm{C},-0.9619505145,0 ., 0.6815481657 \backslash \mathrm{C}, 0.4409726494,0 \ldots, 1$. $1443109591 \backslash \mathrm{C}, 1.2207436843,0 .,-0.129212024 \backslash \mathrm{C}, 0.3567119175,0 .,-1.1662303$ $371 \backslash \mathrm{H},-1.8750831043,0 ., 1.2588787412 \backslash \mathrm{H}, 0.6406154754,0.8755549968,1.7748$ $158451 \backslash \mathrm{H}, 2.2964928179,0 \ldots,-0.1993269525 \backslash \mathrm{H}, 0.4974375994,0 .,-2.2341031585$ $\backslash \mathrm{H},-1.8073552317,0 .,-1.1909645996 \backslash \mathrm{H}, 0.6406154754,-0.8755549968,1.77481$ $58451 \backslash \backslash$ Version $=x 86-$ Linux-G03RevB. 03 \State $=1-A^{\prime} \backslash$ HF $=-209.1742001 \backslash M P 2=-20$ $9.8696305 \backslash \mathrm{RMSD}=8.972 \mathrm{e}-09 \backslash \mathrm{RMSF}=6.311 \mathrm{e}-05 \backslash \mathrm{Dipole}=-0.7933076,0 ., 0.0411742$ $\backslash \mathrm{PG}=\mathrm{CS} \quad[\mathrm{SG}(\mathrm{C} 4 \mathrm{H} 4 \mathrm{~N} 1), \mathrm{X}(\mathrm{H} 2)] \backslash \backslash @$

\section{C4H4NH॰••HF (C3=C4)}

$1 \backslash 1 \backslash \mathrm{GINC}-\mathrm{LC} 94 \backslash \mathrm{FOpt} \backslash \mathrm{RMP} 2-\mathrm{FC} \backslash 6-31+\mathrm{G}(\mathrm{d}, \mathrm{p}) \backslash \mathrm{C} 4 \mathrm{H} 6 \mathrm{~F} 1 \mathrm{~N} 1 \backslash \mathrm{BUC} 562 \backslash 23-\mathrm{Oct}-2004 \backslash 0 \backslash \backslash$ \# MP $2 / 6-31+\mathrm{G}(\mathrm{D}, \mathrm{P})$ OPT MAXDISK $=268435456 \backslash \backslash \mathrm{C} 4 \mathrm{H} 4 \mathrm{NH}-\mathrm{HF} \backslash \backslash 0,1 \backslash \mathrm{N},-0.7742759$ $604,-0.9465657102,-0.5770527233 \backslash \mathrm{C}, 0.5973248064,-0.9883112558,-0.543849$ $1647 \backslash \mathrm{C}, 1.0533339774,0.3190004586,-0.6640621008 \backslash \mathrm{C},-0.0891192025,1.16075$ $37169,-0.770802057 \backslash \mathrm{C},-1.2100885537,0.3494307399,-0.7086710221 \backslash \mathrm{H},-0.094$ $6940725,2.2329851527,-0.8783249946 \backslash \mathrm{H},-2.2600219649,0.5868836976,-0.732$ $6538857 \backslash \mathrm{H},-1.3754559501,-1.7462740148,-0.4543501033 \backslash \mathrm{H}, 2.0876169591,0.6$ $211215496,-0.7072525635 \backslash \mathrm{H}, 1.1279926548,-1.9204663961,-0.4472255985 \backslash \mathrm{H}, 0$ $.566108889,0.2184957685,1.5168536797 \backslash \mathrm{F}, 0.3621865602,0.1764413619,2.429$ $6253995 \backslash \backslash$ Version $=x 86-$ Linux $-G 03$ RevB. $03 \backslash$ State $=1-A \backslash H F=-308.8567659 \backslash M P 2=-3$ $09.7644906 \backslash \mathrm{RMSD}=5.851 \mathrm{e}-09 \backslash \mathrm{RMSF}=1.556 \mathrm{e}-04 \backslash \mathrm{Dipole}=-0.4189632,-0.6173319$, $-0.9516764 \backslash \mathrm{PG}=\mathrm{C} 01 \quad[\mathrm{X}(\mathrm{C} 4 \mathrm{H} 6 \mathrm{~F} 1 \mathrm{~N} 1)] \backslash \backslash @$

\section{$\mathrm{NH}_{2} \mathrm{CHO}$ (Formamide)}

$1 \backslash 1 \backslash$ GINC-LC106 FOpt $\backslash$ RMP $2-F C \backslash 6-31+G(d, p) \backslash C 1 H 3 N 101 \backslash B U C 562 \backslash 22-O c t-2004 \backslash 0 \backslash$ $\backslash \#$ MP2 $/ 6-31+G(D, P)$ OPT MAXDISK $=268435456 \backslash \backslash \mathrm{CH}(\mathrm{O}) \mathrm{NH} 2 \backslash \backslash 0,1 \backslash \mathrm{C}, 0.087881642$, $0.3663553057,-0.1936930003 \backslash 0,1.1687819734,0.1518885052,0.3494406485 \backslash \mathrm{H}$, $-0.0964348807,1.251930658,-0.8181816825 \backslash \mathrm{N},-0.9927927007,-0.4602981072$, $-0.1165990643 \backslash \mathrm{H},-0.9489757134,-1.2608116109,0.4940969817 \backslash \mathrm{H},-1.88258614$ $07,-0.1822721731,-0.4930890356 \backslash \backslash$ Version $=x 86-L$ inux-G03RevB.03 $\backslash$ State $=1-A$ $\backslash \mathrm{HF}=-168.9457436 \backslash \mathrm{MP} 2=-169.4367385 \backslash \mathrm{RMSD}=8.547 \mathrm{e}-09 \backslash \mathrm{RMSF}=4.230 \mathrm{e}-05 \backslash \mathrm{Dipole}$ $=-1.5381482,-0.0726719,-0.3843419 \backslash \mathrm{PG}=\mathrm{C} 01[\mathrm{X}(\mathrm{C} 1 \mathrm{H} 3 \mathrm{~N} 1 \mathrm{O} 1)] \backslash \backslash \mathrm{Q}$

\section{$\mathrm{NH}_{3} \mathrm{CHO}^{+}$}

$1 \backslash 1 \backslash$ GINC-LC13\FOpt \RMP2-FC $66-31+\mathrm{G}(\mathrm{d}, \mathrm{p}) \backslash \mathrm{C} 1 \mathrm{H} 4 \mathrm{~N} 101(1+) \backslash \mathrm{BUC} 562 \backslash 22-\mathrm{OCt}-2004$ $\backslash 0 \backslash \backslash \#$ MP $2 / 6-31+G(D, P) \quad O P T$ MAXDISK $=268435456 \backslash \backslash \mathrm{CH}(\mathrm{O}) \mathrm{NH} 3+\backslash \backslash 1,1 \backslash \mathrm{C}, 0.556774$ $4289,0 .,-0.0344944801 \backslash 0,0.5483729274,0 ., 1.154556363 \backslash \mathrm{H}, 1.3925157112,0 .$, $-0.7363477216 \backslash \mathrm{N},-0.8157502542,0 .,-0.7804054459 \backslash \mathrm{H},-1.5646859335,0,,-0.0$ $77126157 \backslash \mathrm{H},-0.9226039952,-0.8295163949,-1.3765860119 \backslash \mathrm{H},-0.9226039952,0$ $.8295163949,-1.3765860119 \backslash \backslash$ Version $=x 86-$ Linux-G03RevB.03 $\backslash$ State $=1-A^{\prime} \backslash \mathrm{HF}=$ $-169.2463997 \backslash M P 2=-169.7388021 \backslash \mathrm{RMSD}=6.745 e-09 \backslash \mathrm{RMSF}=2.972 \mathrm{e}-05 \backslash \mathrm{Dipole}=-1$. $0524756,0 .,-1.9904373 \backslash P G=C S[S G(C 1 H 2 N 101), X(H 2)] \backslash \backslash @$

\section{$\mathrm{NH}_{2} \mathrm{CHOH}^{+}$}

$1 \backslash 1 \backslash$ GINC-LC2 $\backslash F O p t \backslash R M P 2-F C \backslash 6-31+G(d, p) \backslash C 1 H 4 N 101(1+) \backslash B U C 562 \backslash 22-$ OCt-2004 $0 \backslash \backslash \#$ MP2 $/ 6-31+G(\mathrm{D}, \mathrm{P}) \quad \mathrm{OPT}$ MAXDISK $=268435456 \backslash \backslash \mathrm{CH}(\mathrm{OH}) \mathrm{NH} 2+\backslash \backslash 1,1 \backslash \mathrm{C}, 0,0,0$. $\backslash 0,0 .,-1.29887,0 . \backslash \mathrm{H},-0.4640385799,0.5675484004,-0.7993034281 \backslash \mathrm{N}, 0.57141$ $4221,0.6217782408,0.9842572697 \backslash \mathrm{H}, 1.0104413022,0.10318256,1.7404785544 \backslash$ $\mathrm{H}, 0.5884100658,1.6340814134,1.0135325015 \backslash \mathrm{H},-0.4490409789,-1.6843680541$ ,$-0.7734701581 \backslash \backslash$ Version $=x 86-$ Linux-G03RevB.03 $\backslash$ State $=1-A^{\prime} \backslash \mathrm{HF}=-169.283837$ $8 \backslash M P 2=-169.7632638 \backslash \mathrm{RMSD}=6.279 \mathrm{e}-09 \backslash \mathrm{RMSF}=3.001 \mathrm{e}-05 \backslash \mathrm{Dipole}=0.3819033,0 .,-$ $0.7597912 \backslash \mathrm{PG}=\mathrm{CS}[\mathrm{SG}(\mathrm{C} 1 \mathrm{H} 4 \mathrm{~N} 1 \mathrm{O} 1)] \backslash \backslash @$ 
$\mathrm{NH}_{2} \mathrm{CHO} \cdots \mathrm{HF}$ (N1)

$1 \backslash 1 \backslash$ GINC-LC2 $1 \backslash F O p t \backslash R M P 2-F C \backslash 6-31+G(d, p) \backslash C 1 H 4 F 1 N 101 \backslash B U C 562 \backslash 22-O c t-2004 \backslash 0$ $\backslash \backslash \#$ MP2 $/ 6-31+G(D, P) \quad O P T$ MAXDISK $=268435456 \backslash \backslash \mathrm{CH}(\mathrm{O}) \mathrm{NH} 2 \backslash \backslash 0,1 \backslash \mathrm{C}, 0.525693646$ $2,0.8057664645,-0.6817208789 \backslash 0,1.7283385625,0.6028808451,-0.7369695548$ $\backslash \mathrm{H}, 0.0895128244,1.8031378674,-0.5401720333 \backslash \mathrm{N},-0.4313254086,-0.20917664$ $72,-0.7159214341 \backslash \mathrm{H},-0.0799121288,-1.0768375585,-1.1073675197 \backslash \mathrm{H},-1.3378$ $223329,0.062359987,-1.0773918259 \backslash \mathrm{H},-0.9330920638,-0.6868553828,1.02040$ $08383 \backslash \mathrm{F},-1.3000309795,-0.9216904367,1.8557846991 \backslash \backslash$ Version=x86-Linux-G0 3 RevB.03 $\backslash$ State $=1-A \backslash H F=-268.9743292 \backslash M P 2=-269.6617267 \backslash \mathrm{RMSD}=4.389 \mathrm{e}-09 \backslash \mathrm{RMS}$ $\mathrm{F}=4.306 \mathrm{e}-05 \backslash \mathrm{Dipole}=-0.831246,0.2950465,-1.3792786 \backslash \mathrm{PG}=\mathrm{C} 01 \quad[\mathrm{X}(\mathrm{C} 1 \mathrm{H} 4 \mathrm{~F} 1 \mathrm{~N} 101$ )$] \backslash \backslash @$

\section{$\mathrm{NH}_{2} \mathrm{CHO} \bullet \cdot \mathrm{HF}(\mathrm{O3})$}

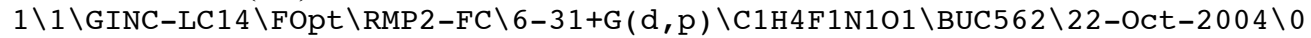
$\backslash \backslash \#$ MP2 $/ 6-31+G(D, P)$ OPT MAXDISK $=268435456 \backslash \backslash \mathrm{CH}(\mathrm{O}) \mathrm{NH} 2-\mathrm{HF} \backslash \backslash 0,1 \backslash \mathrm{C}, 0 ., 0$. , $0 . \backslash 0,0 .,-1.242417,0 . \backslash \mathrm{H},-0.4786503759,0.5615442139,-0.8077697687 \backslash \mathrm{N}, 0.56$ $46343926,0.7667278799,0.9528762865 \backslash \mathrm{H}, 1.029260143,0.3269038664,1.736978$ $1149 \backslash \mathrm{H}, 0.5287069763,1.769527411,0.8922452242 \backslash \mathrm{H}, 0.7804841641,-1.7972429$ $529,1.3171440878 \backslash \mathrm{F}, 1.2701590673,-1.8146861135,2.1435188347 \backslash \backslash$ Version $=\mathrm{x} 8$ $6-$ Linux-G03RevB.03 $\backslash$ State $=1-A^{\prime} \backslash \mathrm{HF}=-268.986065 \backslash \mathrm{MP} 2=-269.6729701 \backslash \mathrm{RMSD}=5.7$ $70 \mathrm{e}-09 \backslash \mathrm{RMSF}=2.221 \mathrm{e}-05 \backslash \mathrm{Dipole}=0.5220381,0 .,-1.9135796 \backslash \mathrm{PG}=\mathrm{CS} \quad[\mathrm{SG}(\mathrm{C} 1 \mathrm{H} 4 \mathrm{~F} 1 \mathrm{~N}$ $101)] \backslash \backslash @$

\section{HOCHCHCHO (Malonaldehyde)}

$1 \backslash 1 \backslash G I N C-L C 87 \backslash F O p t \backslash R M P 2-F C \backslash 6-31+G(d, p) \backslash C 3 H 4 O 2 \backslash$ BUC562 $\backslash 27-O c t-2004 \backslash 0 \backslash \backslash \#$ MP2 $/ 6-31+G(D, P) \quad O P T$ MAXDISK $=268435456 \backslash \backslash \mathrm{CH}(\mathrm{OH}) \mathrm{CHCHO} \backslash \backslash 0,1 \backslash \mathrm{C},-0.886026526$ $4,-0.9443255421,0 . \backslash C, 0.5548313183,-0.9533965134,0 . \backslash C, 1.2429336464,0.22$ $59704415,0 . \backslash 0,0.6705198524,1.4288159437,0 . \backslash 0,-1.5669861501,0.107599079$ $, 0 . \backslash \mathrm{H},-0.3117999545,1.2668780214,0 . \backslash \mathrm{H}, 2.3251629026,0.2713817395,0 . \backslash \mathrm{H}, 1$ $.0942956301,-1.8882249909,0 . \backslash \mathrm{H},-1.4063588259,-1.9108452673,0 . \backslash \backslash$ Version $=x 86-$ Linux $-G 03$ RevB. 03 $\backslash$ State $=1-A^{\prime} \backslash \mathrm{HF}=-265.6473581 \backslash \mathrm{MP} 2=-266.4168055 \backslash \mathrm{RMSD}$ $=5.617 \mathrm{e}-09 \backslash \mathrm{RMSF}=1.352 \mathrm{e}-04 \backslash \mathrm{Dipole}=0.6931115,-0.8715365,0 . \backslash \mathrm{PG}=\mathrm{CS} \quad[\mathrm{SG}(\mathrm{C} 3 \mathrm{H}$ $402)] \backslash \backslash @$

\section{$\mathrm{HOCHCH}_{2} \mathrm{CHO}^{+}$}

$1 \backslash 1 \backslash$ GINC-SC160 \FOpt $\backslash$ RMP $2-F C \backslash 6-31+G(d, p) \backslash C 3 H 5 O 2(1+) \backslash B U C 562 \backslash 28-O c t-2004 \backslash$ $0 \backslash \backslash \#$ MP2 $/ 6-31+G(D, P) \quad O P T$ SYMMETRY $=$ LOOSE MAXDISK $=268435456 \backslash \backslash \mathrm{CH}(\mathrm{OH}) \mathrm{CH} 2 \mathrm{CH}$ $0+\backslash \backslash 1,1 \backslash \mathrm{C}, 1.2662930625,0.2949770975,0 . \backslash \mathrm{C}, 0.0218853654,1.1482956344,0 . \backslash$ $\mathrm{C},-1.2375081759,0.3482419081,0 . \backslash 0,-1.2165597853,-0.9059015139,0 . \backslash 0,1.1$ $889435524,-0.9441993704,0 . \backslash \mathrm{H},-2.2153832556,0.8263877362,0 . \backslash \mathrm{H}, 0.0203351$ $368,1.8198888653,0.8679 \backslash \mathrm{H}, 2.2458579503,0.7763957026,0 . \backslash \mathrm{H},-0.1539426596$ $,-1.1910577449,0 . \backslash \mathrm{H}, 0.0203351368,1.8198888653,-0.8679 \backslash \backslash$ Version=DEC-AXP $-\mathrm{OSF} / 1-\mathrm{G} 03$ RevB.03 $\backslash$ State $=1-\mathrm{A}^{\prime} \backslash \mathrm{HF}=-265.9598824 \backslash \mathrm{MP} 2=-266.7225875 \backslash \mathrm{RMSD}=2.7$ $60 \mathrm{e}-09 \backslash \mathrm{RMSF}=6.271 \mathrm{e}-05 \backslash \mathrm{Dipole}=-0.3418528,1.6146131,0 . \backslash \mathrm{PG}=\mathrm{CS} \quad[\mathrm{SG}(\mathrm{C} 3 \mathrm{H} 3 \mathrm{O} 2)$ $, \mathrm{X}(\mathrm{H} 2)] \backslash \backslash @$

\section{$\mathrm{HOCHCHCHOH}^{+}$}

$1 \backslash 1 \backslash$ GINC-LC87 7 FOpt $\backslash R M P 2-F C \backslash 6-31+G(d, p) \backslash C 3 H 5 O 2(1+) \backslash B U C 562 \backslash 27-O C t-2004 \backslash 0$ $\backslash \backslash \#$ MP $2 / 6-31+G(D, P)$ OPT MAXDISK $=268435456 \backslash \backslash \mathrm{CH}(\mathrm{OH}) \mathrm{CHCHO} \backslash \backslash 1,1 \backslash \mathrm{C},-0.87856$ $13471,-0.9752452438,0 . \backslash C, 0.5257008877,-0.9740183794,0 . \backslash C, 1.284205147,0$ $.1708763458,0 . \backslash 0,0.6931864726,1.3611295063,0 . \backslash 0,-1.6576343071,0.057976$ $2618,0 . \backslash \mathrm{H},-1.1654133838,0.906259685,0 . \backslash \mathrm{H}, 2.3683365052,0.1430211107,0 . \backslash$ $\mathrm{H}, 1.0317144151,-1.9270562186,0 . \backslash \mathrm{H},-1.4290515192,-1.9091608871,0 . \backslash \mathrm{H}, 1.3$ $219285321,2.1044138295,0 . \backslash \backslash$ Version $=x 86-$ Linux-G03RevB.03 $\backslash$ State $=1-A^{\prime} \backslash \mathrm{HF}=$ $-265.9844758 \backslash M P 2=-266.7372315 \backslash \mathrm{RMSD}=5.393 e-09 \backslash \mathrm{RMSF}=4.370 \mathrm{e}-05 \backslash \mathrm{Dipole}=1.1$ $502435,0.1999692,0 . \backslash P G=C S \quad[S G(C 3 H 5 O 2)] \backslash \backslash @$ 


\section{HOCHCHCHO $\cdots H F(O 1)$}

$1 \backslash 1 \backslash$ GINC-LC72 $\backslash F O p t \backslash R M P 2-F C \backslash 6-31+G(d, p) \backslash C 3 H 5 F 102 \backslash B U C 562 \backslash 28-O c t-2004 \backslash 0 \backslash \backslash$

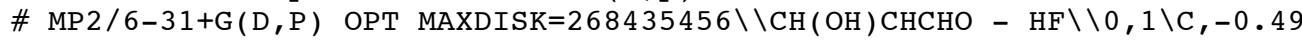
$60428844,0.8098952024,-0.2456589206 \backslash C, 0.6730039782,1.4620040255,-0.491$ $5815829 \backslash \mathrm{C}, 1.9101569541,0.7183789669,-0.5484207756 \backslash 0,1.9661261854,-0.52$ $21986398,-0.3872631145 \backslash 0,-0.570732938,-0.519252743,-0.065247225 \backslash \mathrm{H}, 0.36$ $79721034,-0.8653202164,-0.1343612335 \backslash \mathrm{H}, 2.8385833418,1.269051355,-0.739$ $7235781 \backslash \mathrm{H}, 0.6700816562,2.5319165209,-0.6323437302 \backslash \mathrm{H},-1.4561710613,1.30$ $53320571,-0.1791531371 \backslash \mathrm{H},-1.9165015959,-1.1913832528,0.8601484772 \backslash \mathrm{F},-2$ $.6877576345,-1.4066282855,1.3510537325 \backslash \backslash$ Version=x86-Linux-G03RevB.03 $\backslash \mathrm{S}$ tate $=1-\mathrm{A} \backslash \mathrm{HF}=-365.679469 \backslash \mathrm{MP} 2=-366.6444795 \backslash \mathrm{RMSD}=5.826 \mathrm{e}-09 \backslash \mathrm{RMSF}=4.872 \mathrm{e}-05$ $\backslash$ Dipole $=0.8834947,1.5392963,-0.7066295 \backslash P G=C 01 \quad[X(C 3 H 5 F 102)] \backslash \backslash @$

\section{HOCHCHCHO $\cdots H F(C 3)$}

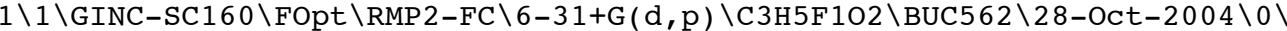
$\backslash \#$ MP2 $/ 6-31+G(D, P)$ OPT MAXDISK $=268435456 \backslash \backslash \mathrm{CH}(\mathrm{OH}) \mathrm{CHCHO}-\mathrm{HF} \backslash \backslash 0,1 \backslash \mathrm{C}, 0.22$ $95645042,1.1619042533,0.4816010015 \backslash \mathrm{C}, 0.0322762433,-0.1166835493,0.9336$ $329,301.85146233957,-1.2126170471,0.3247071583 \backslash 0,1.5057859522,-1.064$ $2103861,-0.6639812085 \backslash 0,0.9955238616,1.4792428391,-0.55380633 \backslash \mathrm{H}, 1.3913$ $591422,0.6258851125,-0.8825232495 \backslash \mathrm{H}, 0.630122024,-2.2138981302,0.754894$ $0646 \backslash \mathrm{H},-0.5553817172,-0.2809715371,1.825791311 \backslash \mathrm{H},-0.2454686462,2.02151$ $29113,0.9395707143 \backslash \mathrm{H},-1.9339115497,-0.1647161582,-0.0873378383 \backslash \mathrm{F},-2.82$ $17091803,-0.2559659738,-0.3608601381 \backslash \backslash$ Version=DEC-AXP-OSF / 1-G03RevB.03 $\backslash$ State $=1-\mathrm{A} \backslash \mathrm{HF}=-365.6726796 \backslash \mathrm{MP} 2=-366.6371256 \backslash \mathrm{RMSD}=8.029 \mathrm{e}-09 \backslash \mathrm{RMSF}=4.304 \mathrm{e}$ $-05 \backslash \mathrm{Dipole}=0.3790167,0.1056244,1.1330451 \backslash \mathrm{PG}=\mathrm{C} 01 \quad[\mathrm{X}(\mathrm{C} 3 \mathrm{H} 5 \mathrm{~F} 102)] \backslash \backslash @$

\section{HOCHCHCHO $\cdots H F(O 5)$}

$1 \backslash 1 \backslash G I N C-L C 84 \backslash F O p t \backslash R M P 2-F C \backslash 6-31+G(d, p) \backslash C 3 H 5 F 102 \backslash B U C 562 \backslash 28-O c t-2004 \backslash 0 \backslash \backslash$

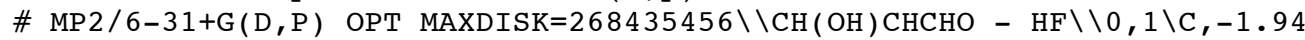
$5497054,0.7998938298,0 . \backslash \mathrm{C},-0.7436308426,1.4554313179,0 . \backslash \mathrm{C}, 0.4810941771$ $, 0.717551754,0 . \backslash 0,0.5185743829,-0.5432001931,0 . \backslash 0,-2.0876526653,-0.519$ $6019786,0 . \backslash \mathrm{H},-1.1751049851,-0.9079199655,0 . \backslash \mathrm{H}, 1.4278171411,1.267890139$ $2,0 . \backslash \mathrm{H},-0.7253690459,2.5341129082,0 . \backslash \mathrm{H},-2.8922302901,1.325822513,0 . \backslash \mathrm{H}$, $2.0840826662,-1.1694147443,0 . \backslash F, 3.0090703433,-1.3761483208,0 . \backslash \backslash$ Version $=x 86-$ Linux-G03RevB.03 $\backslash$ State $=1-A^{\prime} \backslash \mathrm{HF}=-365.6852855 \backslash M P 2=-366.648247 \backslash \mathrm{RMSD}=$ $7.144 \mathrm{e}-09 \backslash \mathrm{RMSF}=5.833 \mathrm{e}-06 \backslash \mathrm{Dipole}=-1.4262662,1.5701894,0 . \backslash \mathrm{PG}=\mathrm{CS} \quad[\mathrm{SG}(\mathrm{C} 3 \mathrm{H} 5$ $\mathrm{F} 102)] \backslash \backslash @$

\section{$\mathrm{C}_{4} \mathrm{H}_{4} \mathrm{O}$ (Furan)}

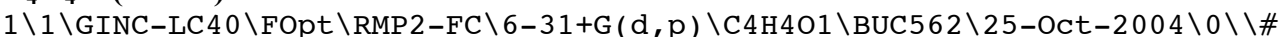
MP2 /6-31+G(D,P) OPT SYMMETRY=LOOSE MAXDISK $=268435456 \backslash \backslash \mathrm{C} 4 \mathrm{H} 4 \mathrm{O} \backslash \backslash 0,1 \backslash \mathrm{O}, 0$. , $0 .,-1.165421 \backslash \mathrm{C}, 0 ., 1.098302,-0.349156 \backslash \mathrm{C}, 0 ., 0.714655,0.964669 \backslash \mathrm{C}, 0 .,-1.09$ $8302,-0.349156 \backslash \mathrm{C}, 0 \ldots,-0.714655,0.964669 \backslash \mathrm{H}, 0 .,-2.050711,-0.849061 \backslash \mathrm{H}, 0 .,-$ $1.372519,1.817665 \backslash \mathrm{H}, 0.1 .372519,1.817665 \backslash \mathrm{H}, 0.2 .050711,-0.849061 \backslash \backslash$ Vers ion $=x 86-L$ inux $-G 03 R e v B .03 \backslash$ State $=1-A 1 \backslash \mathrm{HF}=-228.6376098 \backslash \mathrm{MP} 2=-229.3564467 \backslash \mathrm{R}$ $\mathrm{MSD}=4.963 \mathrm{e}-09 \backslash \mathrm{RMSF}=1.421 \mathrm{e}-05 \backslash \mathrm{Dipole}=0.2885961,0 ., 0 . \backslash \mathrm{PG}=\mathrm{C} 02 \mathrm{~V} \quad[\mathrm{C} 2(\mathrm{O} 1), \mathrm{SG}$ $\mathrm{V}(\mathrm{C} 4 \mathrm{H} 4) \mathrm{J} \backslash \backslash \mathrm{Q}$

\section{$\mathrm{C}_{4} \mathrm{H}_{4} \mathrm{OH}^{+}$}

$1 \backslash 1 \backslash$ GINC-LC56 $\backslash$ FOpt $\backslash$ RMP 2-FC $\backslash 6-31+\mathrm{G}(\mathrm{d}, \mathrm{p}) \backslash \mathrm{C} 4 \mathrm{H} 5 \mathrm{O} 1(1+) \backslash \mathrm{BUC} 562 \backslash 25-$ Oct $-2004 \backslash 0$ $\backslash \backslash \#$ MP2 $/ 6-31+G(D, P)$ OPT SYMMETRY=LOOSE MAXDISK $=268435456 \backslash \backslash C 4 \mathrm{H} 4 \mathrm{OH}+\backslash \backslash 1,1$ $\backslash 0,-0.072231,-1.1148170882,-0.005569412 \backslash \mathrm{C}, 0.004996,-0.2634854953,-1.17$ $45579636 \backslash C, 0.011324,1.0009215102,-0.7200166358 \backslash C, 0.004996,-0.275207782$ $4,1.1718667555 \backslash \mathrm{C}, 0.011324,0.9936775992,0.7299812697 \backslash \mathrm{H}, 0.429937,-1.9589$ $845539,-0.0097867105 \backslash \mathrm{H},-0.031563,-0.786220652,-2.1146311464 \backslash \mathrm{H}, 0.007598$ $, 1.8746088314,-1.3516377981 \backslash \mathrm{H}, 0.007598,1.8610105519,1.3703002349 \backslash \mathrm{H},-0$. $031563,-0.8073094624,2.1066701761 \backslash \backslash$ Version=x86-Linux-G03RevB.03 $\backslash$ State $=$ $1-\mathrm{A}^{\prime} \backslash \mathrm{HF}=-228.921169 \backslash \mathrm{MP} 2=-229.6270487 \backslash \mathrm{RMSD}=3.743 \mathrm{e}-09 \backslash \mathrm{RMSF}=7.971 \mathrm{e}-05 \backslash \mathrm{Dip}$ ole $=0 .,-0.9569777,0.3337298 \backslash \mathrm{PG}=\mathrm{CS}[\mathrm{SG}(\mathrm{H} 1 \mathrm{O} 1), \mathrm{X}(\mathrm{C} 4 \mathrm{H} 4)] \backslash \backslash @$ 


\section{$\mathrm{C}_{4} \mathrm{H}_{5} \mathrm{O}^{+}(\mathrm{C} 2)$}

$1 \backslash 1 \backslash$ GINC-LC4 2\FOpt \RMP2-FC $\backslash 6-31+\mathrm{G}(\mathrm{d}, \mathrm{p}) \backslash \mathrm{C} 4 \mathrm{H} 5 \mathrm{O} 1(1+) \backslash \mathrm{BUC} 562 \backslash 24-\mathrm{Oct}-2004 \backslash 0$

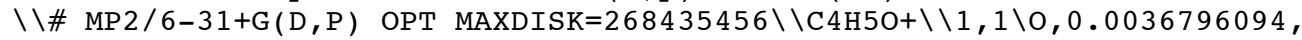
$1.1787039229,0 . \backslash C,-1.1541193288,0.2795201264,0 . \backslash C,-0.553121957,-1.0692$ $579979,0 . \backslash C, 0.8055017956,-0.949765179,0 . \backslash C, 1.0685596287,0.4485773274,0$ . $\backslash \mathrm{H},-1.7326213406,0.5244096403,0.893659748 \backslash \mathrm{H}, 1.5521641656,-1.727467747$ $, 0 . \backslash \mathrm{H}, 2.0136455893,0.97758211,0 . \backslash \mathrm{H},-1.1309247793,-1.9830106881,0 . \backslash \mathrm{H},-1$ $.7326213406,0.5244096403,-0.893659748 \backslash \backslash$ Version=x86-Linux-G03RevB.03\St ate $=1-A^{\prime} \backslash \mathrm{HF}=-228.9768272 \backslash M P 2=-229.6732658 \backslash \mathrm{RMSD}=3.596 \mathrm{e}-09 \backslash \mathrm{RMSF}=7.485 \mathrm{e}-0$ $5 \backslash$ Dipole $=0.0191917,-0.4987143,0 . \backslash P G=C S \quad[S G(C 4 H 3 O 1), x(H 2)] \backslash \backslash @$

\section{$\mathrm{C}_{4} \mathrm{H}_{5} \mathrm{O}^{+}(\mathrm{C} 3)$}

$1 \backslash 1 \backslash$ GINC-LC146\FOpt \RMP2-FC $\backslash 6-31+G(d, p) \backslash C 4 H 5 O 1(1+) \backslash B U C 562 \backslash 24-$ Oct-2004 $0 \backslash \backslash \#$ MP2 $/ 6-31+G(D, P)$ OPT MAXDISK $=268435456 \backslash \backslash C 4 \mathrm{H} 5 \mathrm{O}+\backslash \backslash 1,1 \backslash 0,-0.013880497$ $7,1.189949972,0 . \backslash \mathrm{C},-1.0570933583,0.454176908,0 . \backslash \mathrm{C},-0.7188878771,-0.971$ $5929765,0 . \backslash C, 0.773151049,-0.9356889869,0 . \backslash C, 1.1601736236,0.3457718846$, $0 . \backslash \mathrm{H},-2.0170126627,0.9559158901,0 . \backslash \mathrm{H},-1.1626053773,-1.4679270861,0.874$ $4884148 \backslash \mathrm{H}, 1.4257868365,-1.7937295543,0 . \backslash \mathrm{H}, 2.0834199399,0.8980670854,0$. $\backslash \mathrm{H},-1.1626053773,-1.4679270861,-0.8744884148 \backslash \backslash$ Version=x86-Linux-G03Rev B. $03 \backslash$ State $=1-A^{\prime} \backslash \mathrm{HF}=-228.9532458 \backslash \mathrm{MP} 2=-229.6535882 \backslash \mathrm{RMSD}=6.494 \mathrm{e}-09 \backslash \mathrm{RMSF}=1$ $.056 \mathrm{e}-04 \backslash \mathrm{Dipole}=-0.633074,-0.4343582,0 . \backslash \mathrm{PG}=\mathrm{CS} \quad[\mathrm{SG}(\mathrm{C} 4 \mathrm{H} 3 \mathrm{O} 1), \mathrm{X}(\mathrm{H} 2)] \backslash \backslash @$

\section{$\mathrm{C}_{4} \mathrm{H}_{4} \mathrm{O} \cdots \mathrm{HF}(\mathrm{O1})$}

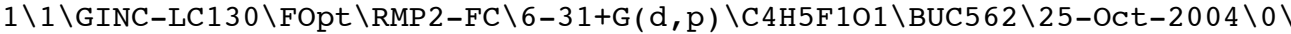
$\backslash \#$ MP2 $/ 6-31+G(D, P)$ OPT SYMMETRY $=$ LOOSE MAXDISK $=268435456 \backslash \backslash C 4 \mathrm{H} 4 \mathrm{O}-\mathrm{HF}(\mathrm{O}) \backslash$ $\backslash 0,1 \backslash 0,0 ., 0.3387145518,0 . \backslash \mathrm{C}, 1.1067398506,-0.4783833746,0 . \backslash \mathrm{C}, 0.71564365$ $96,-1.7861321263,0 . \backslash C,-1.1067398506,-0.4783833746,0 . \backslash C,-0.7156436596,-$ $1.7861321263,0 . \backslash \mathrm{H}, 2.0573453099,0.0246327657,0 . \backslash \mathrm{H}, 1.370085184,-2.641637$ $9082,0 . \backslash \mathrm{H},-1.370085184,-2.6416379082,0 . \backslash \mathrm{H},-2.0573453099,0.0246327657,0$ $. \backslash \mathrm{H}, 0 ., 2.1259741264,0 . \backslash \mathrm{F}, 0 ., 3.0636117506,0 . \backslash \backslash$ Version=x86-Linux-G03RevB $.03 \backslash$ State $=1-\mathrm{A} 1 \backslash \mathrm{HF}=-328.6700026 \backslash \mathrm{MP} 2=-329.5832496 \backslash \mathrm{RMSD}=6.243 \mathrm{e}-09 \backslash \mathrm{RMSF}=1$. $035 \mathrm{e}-04 \backslash \mathrm{Dipole}=0 .,-1.4956559,0 . \backslash \mathrm{PG}=\mathrm{C} 02 \mathrm{~V} \quad[\mathrm{C} 2(\mathrm{O} 1 \mathrm{H} 1 \mathrm{~F} 1), \mathrm{SGV}(\mathrm{C} 4 \mathrm{H} 4)] \backslash \backslash @$

\section{$\mathrm{C}_{4} \mathrm{H}_{4} \mathrm{O} \cdots \cdot \cdot \mathrm{HF}(\mathrm{C} 3)$}

$1 \backslash 1 \backslash$ GINC-LC22 2 FOpt $\backslash$ RMP 2-FC $\backslash 6-31+G(d, p) \backslash C 4 H 5 F 101 \backslash B U C 562 \backslash 24-O C t-2004 \backslash 0 \backslash \backslash$ \# MP2 /6-31+G(D,P) OPT MAXDISK $=268435456 \backslash \backslash C 4 \mathrm{H} 4 \mathrm{O}-\mathrm{HF}(\mathrm{C}) \backslash \backslash 0,1 \backslash \mathrm{O}, 0.9157408$ $132,-1.0723581071,-0.3421961463 \backslash \mathrm{C}, 0.4400345969,-0.7622944627,0.8998799$ $895 \backslash \mathrm{C}, 0.2925549052,0.5965767928,1.0254525852 \backslash \mathrm{C}, 0.7095340393,1.16160906$ $59,-0.2219128687 \backslash \mathrm{C}, 1.0776091953,0.1083159321,-1.0147838607 \backslash \mathrm{H}, 0.7305278$ $873,2.2027613662,-0.4968288569 \backslash \mathrm{H}, 1.4408549718,0.0242456762,-2.02354265$ $92 \backslash \mathrm{H},-0.0416530838,1.1197113327,1.9067004427 \backslash \mathrm{H}, 0.266722257,-1.59568616$ $48,1.5578893534 \backslash \mathrm{H},-1.707139637,0.127621192,0.067789661 \backslash \mathrm{F},-2.5704039246$ $, 0.0083297207,-0.267361538 \backslash \backslash$ Version $=x 86-$ Linux-G03RevB. $03 \backslash$ State $=1-\mathrm{A} \backslash \mathrm{HF}=$ $-328.664775 \backslash M P 2=-329.5796334 \backslash R M S D=3.894 e-09 \backslash R M S F=5.240 e-05 \backslash D i p o l e=0.94$ $20235,0.3408738,0.359911 \backslash P G=C 01 \quad[X(C 4 H 5 F 101)] \backslash \backslash @$

\section{C4H6 (1,3-Butadiene)}

$1 \backslash 1 \backslash$ GINC-LC1 40 $\backslash$ FOpt $\backslash R M P 2-F C \backslash 6-31+G(d, p) \backslash C 4 H 6 \backslash B U C 562 \backslash 26-O c t-2004 \backslash 0 \backslash \backslash \# M$ $\mathrm{P} 2 / 6-31+\mathrm{G}(\mathrm{D}, \mathrm{P})$ OPT $\mathrm{MAXDISK}=268435456 \backslash \backslash \mathrm{C} 4 \mathrm{H} 6$ trans $\backslash \backslash 0,1 \backslash \mathrm{C},-1.7457724539$, $-0.6216874077,0 . \backslash C,-0.3988876858,-0.6101647388,0 . \backslash C, 0.3988876858,0.610$ $1647388,0 . \backslash \mathrm{C}, 1.7457724539,0.6216874077,0 . \backslash \mathrm{H}, 2.3022117868,1.5481264908$, $0 . \backslash \mathrm{H}, 2.3135991545,-0.3003094753,0 . \backslash \mathrm{H},-0.144252229,1.5508293526,0 . \backslash \mathrm{H}, 0$. $144252229,-1.5508293526,0 . \backslash \mathrm{H},-2.3022117868,-1.5481264908,0 . \backslash \mathrm{H},-2.31359$ $91545,0.3003094753,0 . \backslash \backslash$ Version $=x 86-$ Linux-G03RevB . 03 $\backslash$ State $=1-A G \backslash H F=-154$ $.9357922 \backslash M P 2=-155.4817365 \backslash \mathrm{RMSD}=9.897 \mathrm{e}-09 \backslash \mathrm{RMSF}=1.001 \mathrm{e}-04 \backslash \mathrm{Dipole}=0 ., 0 ., 0$ $. \backslash \mathrm{PG}=\mathrm{C} 02 \mathrm{H} \quad[\mathrm{SGH}(\mathrm{C} 4 \mathrm{H} 6)] \backslash \backslash @$

\section{$\mathrm{C}_{4} \mathrm{H}_{7}^{+}$(C1)}

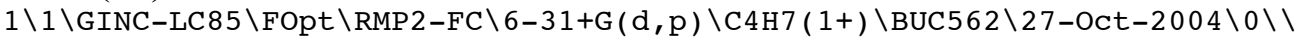
\# MP2 $/ 6-31+G(D, P)$ OPT MAXDISK $=268435456 \backslash \backslash \mathrm{CH} 3 \mathrm{CHCHCH} 2 \backslash \backslash 1,1 \backslash \mathrm{C},-1.09842661$ $82,-1.4865338208,0 . \backslash \mathrm{C},-0.579732365,-0.1231341075,0 . \backslash \mathrm{C}, 0.7740876479,0.2$ $278016985,0 . \backslash \mathrm{C}, 1.0977655025,1.562838177,0 . \backslash \mathrm{H}, 2.1273658859,1.8977677345$ $, 0 . \backslash \mathrm{H}, 0.325397503,2.3234755466,0 . \backslash \mathrm{H}, 1.5402700956,-0.5362688892,0 . \backslash \mathrm{H},-1$ $.3058403047,0.6878699762,0 . \backslash \mathrm{H},-1.7617922465,-1.6049003334,0.866 \backslash \mathrm{H},-1.7$ $617922465,-1.6049003334,-0.866 \backslash \mathrm{H},-0.3267156626,-2.2489913834,0 . \backslash \backslash$ Versi on $=x 86-$ Linux-G03RevB.03 $\backslash$ State $=1-A^{\prime} \backslash H F=-155.2662459 \backslash M P 2=-155.7933152 \backslash$ RM $\mathrm{SD}=7.160 \mathrm{e}-09 \backslash \mathrm{RMSF}=1.079 \mathrm{e}-04 \backslash \mathrm{Dipole}=-0.4246449,-0.2909398,0 . \backslash \mathrm{PG}=\mathrm{CS} \quad[\mathrm{SG}($ $\mathrm{C} 4 \mathrm{H} 5), \mathrm{X}(\mathrm{H} 2) \mathrm{J} \backslash \backslash @$ 


\section{$\mathrm{C}_{4} \mathrm{H}_{6} \cdots \bullet \mathrm{HF}(\mathrm{C} 1=\mathrm{C} 2)$}

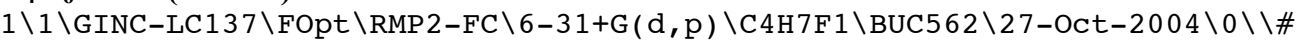

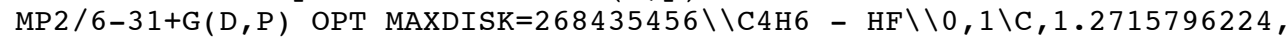
$0.3680719287,-1.0432769335 \backslash C, 0.0540567292,-0.1774274948,-0.8349213498 \backslash$ $\mathrm{C},-1.1186831163,0.5841394872,-0.4205885689 \backslash \mathrm{C},-2.3200996096,0.019656023$ $9,-0.1961614611 \backslash \mathrm{H},-3.171541098,0.6102317264,0.1104203189 \backslash \mathrm{H},-2.47038582$ $86,-1.0459607075,-0.3154620991 \backslash \mathrm{H},-0.9927629601,1.6546931411,-0.2907102$ $777 \backslash \mathrm{H},-0.0830975996,-1.2450800278,-0.9850431986 \backslash \mathrm{H}, 1.338497585,-0.36958$ $11289,1.1007770185 \backslash \mathrm{H}, 2.1099559184,-0.2326257471,-1.3680964462 \backslash \mathrm{H}, 1.4394$ $032339,1.4306224438,-0.9165054884 \backslash F, 1.6120898884,-0.6187710411,1.95936$ $77836 \backslash \backslash$ Version $=x 86-$ Linux-G03RevB $.03 \backslash$ State $=1-A \backslash H F=-254.9636377 \backslash M P 2=-255$ $.705084 \backslash \mathrm{RMSD}=7.551 \mathrm{e}-09 \backslash \mathrm{RMSF}=2.820 \mathrm{e}-06 \backslash \mathrm{Dipole}=-0.4631857,0.2586495,-0.9$ $265638 \backslash P G=C 01 \quad[X(C 4 H 7 F 1)] \backslash \backslash @$

\section{HOCHO (Formic Acid)}

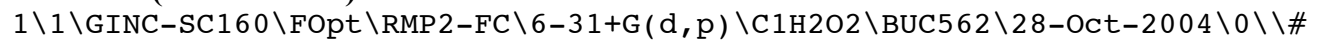
MP $2 / 6-31+G(D, P)$ OPT MAXDISK $=268435456 \backslash \backslash \mathrm{HCO} 2 \mathrm{H} \backslash \backslash 0,1 \backslash \mathrm{C},-0.3038287026,-0$. $2978596751,0 . \backslash 0,1.0417746856,-0.4388826393,0 . \backslash 0,-0.8943960622,0.765261$ $2081,0 . \backslash \mathrm{H},-0.7772569071,-1.2814588452,0 . \backslash \mathrm{H}, 1.4212001352,0.4575883457,0$ . $\backslash \backslash$ Version=DEC-AXP-OSF $/ 1-G 03 R e v B .03 \backslash$ State $=1-A^{\prime} \backslash \mathrm{HF}=-188.7736337 \backslash \mathrm{MP} 2=-18$ $9.2753342 \backslash \mathrm{RMSD}=7.831 \mathrm{e}-09 \backslash \mathrm{RMSF}=3.593 \mathrm{e}-05 \backslash \mathrm{Dipole}=0.4470259,-0.3120994,0$. $\backslash \mathrm{PG}=\mathrm{CS}[\mathrm{SG}(\mathrm{C} 1 \mathrm{H} 2 \mathrm{O} 2)] \backslash \backslash @$

\section{$\mathrm{H}_{2} \mathrm{OCHO}^{+}$}

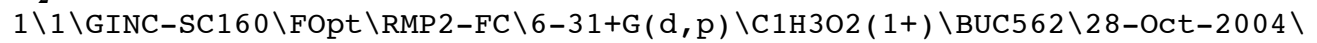
$0 \backslash \backslash \#$ MP2 $/ 6-31+G(D, P)$ OPT MAXDISK $=268435456 \backslash \backslash \mathrm{HCO} 2 \mathrm{H}+\backslash \backslash 1,1 \backslash \mathrm{C}, 0.7864089117$ $,-0.1573183094,0 . \backslash 0,-0.9279494482,0.9781757529,0 . \backslash 0,0.5229645702,-1.26$ $54920617,0 . \backslash \mathrm{H}, 1.3884314529,0.7403056127,0 . \backslash \mathrm{H},-1.8054086662,0.562672363$ $, 0 . \backslash \mathrm{H},-1.0615996296,1.9394668519,0 . \backslash \backslash$ Version=DEC-AXP-OSF / 1-G03RevB .03 State $=1-A^{\prime} \backslash H F=-189.0287395 \backslash M P 2=-189.5373381 \backslash R M S D=1.754 \mathrm{e}-09 \backslash \mathrm{RMSF}=4.437 \mathrm{e}$ $-05 \backslash \mathrm{Dipole}=-0.4957803,0.890585,0 . \backslash \mathrm{PG}=\mathrm{CS}[\mathrm{SG}(\mathrm{C} 1 \mathrm{H} 3 \mathrm{O} 2)] \backslash \backslash @$

\section{$\mathrm{HOCHOH}^{+}$}

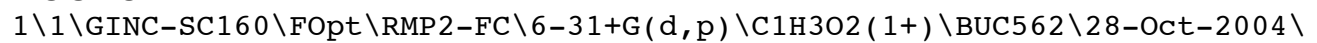
$0 \backslash \backslash \#$ MP2 $/ 6-31+\mathrm{G}(\mathrm{D}, \mathrm{P})$ OPT MAXDISK $=268435456 \backslash \backslash \mathrm{HCO} 2 \mathrm{H}+\backslash \backslash 1,1 \backslash \mathrm{C},-0.229696296$ $9,-0.3643593924,0 . \backslash 0,1.0503495323,-0.3109233181,0 . \backslash 0,-0.9184257343,0.7$ $035157846,0 . \backslash \mathrm{H},-0.7868515144,-1.2961807569,0 . \backslash \mathrm{H}, 1.4901714177,-1.185061$ $521,0 . \backslash \mathrm{H},-0.380532506,1.5266589,0 . \backslash \backslash$ Version=DEC-AXP-OSF $/ 1-G 03$ RevB.03 $\backslash \mathrm{S}$ tate $=1-A^{\prime} \backslash \mathrm{HF}=-189.076745 \backslash M P 2=-189.5646332 \backslash \mathrm{RMSD}=8.651 \mathrm{e}-09 \backslash \mathrm{RMSF}=6.931 \mathrm{e}-0$ $5 \backslash$ Dipole $=0.3786749,-0.6952033,0 . \backslash P G=C S[S G(C 1 H 3 O 2)] \backslash \backslash @$

\section{HOCHO $\bullet$ HF (O1)}

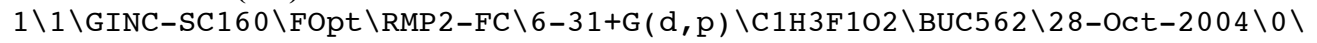
$\backslash \#$ MP2 $/ 6-31+G(D, P) \quad O P T$ MAXDISK $=268435456 \backslash \backslash \mathrm{HCO} 2 \mathrm{H}-\mathrm{HF} \backslash \backslash 0,1 \backslash \mathrm{C}, 1.07133794$ $89,-0.3721871471,0.097266 \backslash 0,0.0263270701,0.5104749964,0.141549 \backslash 0,2.212$ $789992,-0.0584093039,-0.15571 \backslash \mathrm{H}, 0.7128298109,-1.3768280979,0.325707 \backslash \mathrm{H}$, $0.3738351915,1.3943229487,-0.076466 \backslash \mathrm{H},-1.7400029858,0.103112239,0.0044$ $72 \backslash \mathrm{F},-2.631959023,-0.1671116385,-0.080446 \backslash \backslash$ Version=DEC-AXP-OSF $/ 1-G 03 \mathrm{Re}$ vB. 03 \State $=1-A \backslash H F=-288.8039762 \backslash M P 2=-289.5004417 \backslash \mathrm{RMSD}=6.944 \mathrm{e}-09 \backslash \mathrm{RMSF}=1$ $.084 \mathrm{e}-04 \backslash \mathrm{Dipole}=0.575133,0.3011223,0.1409898 \backslash \mathrm{PG}=\mathrm{C} 01[\mathrm{X}(\mathrm{C} 1 \mathrm{H} 3 \mathrm{~F} 102)] \backslash \backslash @$

\section{HOCHO $\bullet$ HF (O3)}

$1 \backslash 1 \backslash$ GINC-SC10 $1 \backslash F O p t \backslash R M P 2-F C \backslash 6-31+G(d, p) \backslash C 1 H 3 F 102 \backslash B U C 562 \backslash 28-O C t-2004 \backslash 0 \backslash$ $\backslash \#$ MP2 /6-31+G(D,P) OPT MAXDISK $=268435456 \backslash \backslash \mathrm{HCO} 2 \mathrm{H}-\mathrm{HF} \backslash \backslash 0,1 \backslash \mathrm{C},-0.4138645$ $529,-1.0765515212,0 . \backslash 0,0.9169117049,-0.9843440115,0 . \backslash 0,-1.2012395108,-$ $0.1328655908,0 . \backslash \mathrm{H},-0.733689586,-2.1177910273,0 . \backslash \mathrm{H}, 1.1716624153,-0.0375$ $969725,0 . \backslash \mathrm{H},-0.3150102072,1.3340256031,0 . \backslash \mathrm{F}, 0.5149830158,1.8020387047$, $0 . \backslash \backslash$ Version=DEC-AXP-OSF $/ 1-G 03 R e v B .03 \backslash$ State $=1-A^{\prime} \backslash H F=-288.8107589 \backslash M P 2=-2$ $89.5077182 \backslash \mathrm{RMSD}=7.222 \mathrm{e}-09 \backslash \mathrm{RMSF}=1.043 \mathrm{e}-05 \backslash \mathrm{Dipole}=-0.0809866,-0.8383788$, $0 . \backslash P G=C S[S G(C 1 H 3 F 102)] \backslash \backslash @$ 


\section{$\mathrm{NH}_{2} \mathrm{CHCH}_{2} \bullet \bullet \mathrm{H}_{2} \mathrm{~F}^{+}$(N1)}

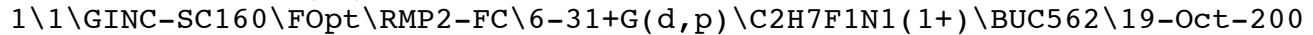
$4 \backslash 0 \backslash \backslash \#$ MP2 $/ 6-31+G(D, P)$ OPT MAXDISK $=134217728 \backslash \backslash \mathrm{CH} 2 \mathrm{CHNH} 2-\mathrm{H} 2 \mathrm{~F} \backslash \backslash 1,1 \backslash \mathrm{C}, 0$. $9482131851,-0.2418439963,0.3874971976 \backslash \mathrm{C}, 2.0869613042,-0.4880519766,-0$. $2529433437 \backslash \mathrm{N}, 0.1295373127,0.9399707942,0.0405409827 \backslash \mathrm{H}, 0.5162537883,-0$. $8391819843,1.1754978888 \backslash \mathrm{H}, 2.4708185244,0.1461096035,-1.041146189 \backslash \mathrm{H}, 2.6$ $63608413,-1.3592326534,0.0179597704 \backslash \mathrm{H}, 0.0507254407,1.5881729853,0.8302$ $104562 \backslash \mathrm{H}, 0.5394632581,1.4562042217,-0.7422074952 \backslash \mathrm{H},-0.8254000405,0.658$ $782031,-0.220713338 \backslash \mathrm{F},-2.3662434922,-0.3612542852,-0.0874964481 \backslash \mathrm{H},-3.2$ $370860789,-0.5999853592,-0.3232430623 \backslash \backslash$ Version=DEC-AXP-OSF / 1-G03RevB. 0 $3 \backslash$ State $=1-A \backslash H F=-233.4691579 \backslash M P 2=-234.1115979 \backslash \mathrm{RMSD}=7.651 \mathrm{e}-09 \backslash \mathrm{RMSF}=2.963$ e-05\Dipole $=-0.24443,1.0441248,-0.1943768 \backslash P G=C 01 \quad[X(C 2 H 7 F 1 N 1)] \backslash \backslash @$

\section{$\mathrm{NH}_{2} \mathrm{CHCH}_{2} \cdots \bullet_{2} \mathrm{H}_{2} \mathrm{~F}^{+}(\mathrm{C} 3)$}

$1 \backslash 1 \backslash$ GINC-LC88\FOpt \RMP2-FC $\backslash 6-31+G(d, p) \backslash C 2 H 7 F 1 N 1(1+) \backslash B U C 562 \backslash 02-N o v-2004$ $\backslash 0 \backslash \backslash \#$ MP $2 / 6-31+G(D, P) \quad O P T$ SYMMETRY $=$ LOOSE MAXDISK $=1342177280 \backslash \backslash \mathrm{CH} 2 \mathrm{CHNH} 2$ - $\mathrm{H} 2 \mathrm{~F} \backslash \backslash 1,1 \backslash \mathrm{C},-0.3789394231,0 ., 2.1786414117 \backslash \mathrm{C},-0.238845542,0 ., 0.7115371$ $802 \backslash \mathrm{N}, 0.8949629304,0 ., 0.0982041853 \backslash \mathrm{H}, 0.5785768064,0 ., 2.6923162008 \backslash \mathrm{H},-0$ $.9606112524,0.8758740459,2.4727577951 \backslash \mathrm{H},-0.9606112524,-0.8758740459,2$. $4727577951 \backslash \mathrm{H},-1.1168900417,0 ., 0.0749477862 \backslash \mathrm{H}, 1.7730580082,0 ., 0.6092700$ $923 \backslash \mathrm{H}, 0.9256445641,0 .,-0.9202577157 \backslash \mathrm{F},-0.2679908498,0 .,-2.4504870622 \backslash \mathrm{H}$ $,-0.3852799061,0 .,-3.3759092426 \backslash \backslash$ Version $=x 86-L i n u x-G 03$ RevB.03 $\backslash$ State $=1-$ $A^{\prime} \backslash H F=-233.4975255 \backslash M P 2=-234.1337343 \backslash R M S D=7.209 e-09 \backslash R M S F=8.523 e-05 \backslash D i p o$ $l e=0.437737,0 ., 0.1141869 \backslash \mathrm{PG}=\mathrm{CS} \quad[\mathrm{SG}(\mathrm{C} 2 \mathrm{H} 5 \mathrm{~F} 1 \mathrm{~N} 1), \mathrm{X}(\mathrm{H} 2)] \backslash \backslash \varrho$

\section{$\mathrm{NH}_{2} \mathrm{CHCH}_{2} \cdots \mathrm{H}_{2} \mathrm{Cl}^{+}(\mathrm{N} 1)$}

$1 \backslash 1 \backslash$ GINC-LC15 $\backslash F O p t \backslash R M P 2-F C \backslash 6-31+G(d, p) \backslash C 2 H 7 C 11 N 1(1+) \backslash B U C 562 \backslash 08-N o v-200$ $4 \backslash 0 \backslash \backslash \#$ MP2 /6-31+G(D,P) OPT MAXDISK $=1342177280 \backslash \backslash \mathrm{CH} 2 \mathrm{CHNH} 2-\mathrm{H} 2 \mathrm{Cl} \backslash \backslash 1,1 \backslash \mathrm{C}$, $1.5852921892,0.0602136782,0.6494350154 \backslash \mathrm{C}, 2.6430762551,-0.2249840061,-0$ $.1040384894 \backslash \mathrm{N}, 0.6529137883,1.1401003099,0.2611045043 \backslash \mathrm{H}, 1.3002379441,-0$ $.4406898535,1.5620286567 \backslash \mathrm{H}, 2.8809894293,0.3083627861,-1.0149951079 \backslash \mathrm{H}, 3$ $.3023346627,-1.0243938583,0.1983064988 \backslash \mathrm{H}, 0.5820798888,1.8571892328,0.9$ $894920646 \backslash \mathrm{H}, 0.961483564,1.6021077628,-0.5989569503 \backslash \mathrm{H},-0.294604657,0.76$ $44893167,0.0931479276 \backslash \mathrm{Cl},-2.0862996293,-0.5910896247,-0.3619144469 \backslash \mathrm{H},-$ $3.2060343174,-0.0106219683,-0.1765881785 \backslash \backslash$ Version=x86-Linux-G03RevB. 03 $\backslash$ State $=1-\mathrm{A} \backslash \mathrm{HF}=-593.5039217 \backslash \mathrm{MP} 2=-594.0973491 \backslash \mathrm{RMSD}=5.803 \mathrm{e}-09 \backslash \mathrm{RMSF}=4.390 \mathrm{e}$ $-06 \backslash \mathrm{Dipole}=0.8905523,1.8257069,0.4274155 \backslash \mathrm{PG}=\mathrm{C} 01 \quad[\mathrm{X}(\mathrm{C} 2 \mathrm{H} 7 \mathrm{C} 11 \mathrm{~N} 1)] \backslash \backslash @$

\section{$\mathrm{NH}_{2} \mathrm{CHCH}_{2} \bullet \bullet \mathrm{H}_{2} \mathrm{Cl}^{+}(\mathrm{C} 3)$}

$1 \backslash 1 \backslash$ GINC-LC $11 \backslash$ FOpt $\backslash$ RMP $2-F C \backslash 6-31+G(d, p) \backslash C 2 H 7 C l 1 N 1(1+) \backslash B U C 562 \backslash 08-N o v-200$ $4 \backslash 0 \backslash \backslash \#$ MP2 $/ 6-31+G(D, P)$ OPT MAXDISK $=1342177280 \backslash \backslash \mathrm{CH} 2 \mathrm{CHNH} 2-\mathrm{H} 2 \mathrm{~F} \backslash \backslash 1,1 \backslash \mathrm{C}, 2$ $.6211050459,-1.4821527629,-0.1561618024 \backslash \mathrm{C}, 1.257579316,-0.9249043944,-0$ $.1353910203 \backslash \mathrm{N}, 0.9958253057,0.3347344453,-0.0518703413 \backslash \mathrm{H}, 3.3895573777,-$ $0.7161422051,-0.0954495769 \backslash \mathrm{H}, 2.7243108667,-2.1811381966,0.6760324758 \backslash \mathrm{H}$ $, 2.7458306484,-2.0648207683,-1.0708033842 \backslash \mathrm{H}, 0.3979675673,-1.5847069263$ $,-0.1922779059 \backslash \mathrm{H}, 1.7404011527,1.0241644064,0.0096682243 \backslash \mathrm{H}, 0.0299315318$ $, 0.6706260117,-0.0363241969 \backslash \mathrm{Cl},-2.2618675611,0.9134071469,0.2137783882$ $\backslash \mathrm{H},-2.8191339167,1.4232980075,-0.8126689108 \backslash \backslash$ Version=x86-Linux-G03RevB $.03 \backslash$ State $=1-\mathrm{A} \backslash \mathrm{HF}=-593.5321279 \backslash \mathrm{MP} 2=-594.1193096 \backslash \mathrm{RMSD}=7.792 \mathrm{e}-09 \backslash \mathrm{RMSF}=5.8$ $62 \mathrm{e}-06 \backslash \mathrm{Dipole}=1.8603008,-0.3400887,-0.6418562 \backslash \mathrm{PG}=\mathrm{C} 01 \quad[\mathrm{X}(\mathrm{C} 2 \mathrm{H} 7 \mathrm{C} 11 \mathrm{~N} 1)] \backslash \backslash @$

\section{$\mathrm{NH}_{2} \mathrm{CHCH}_{2} \bullet \bullet \mathrm{H}_{2} \mathrm{Br}^{+}(\mathrm{N} 1)$}

$1 \backslash 1 \backslash G I N C-L C 38 \backslash F O p t \backslash R M P 2-F C \backslash 6-31+G(d, p) \backslash C 2 H 7 B r 1 N 1(1+) \backslash B U C 562 \backslash 08-N o v-200$ $4 \backslash 0 \backslash \backslash \#$ MP2 $/ 6-31+G(D, P) \quad O P T$ MAXDISK $=1342177280 \backslash \backslash \mathrm{CH} 2 \mathrm{CHNH} 2-\mathrm{H} 2 \mathrm{Br}+\backslash \backslash 1,1 \backslash \mathrm{C}$ $, 2.0361032872,0.407456831,0.7950473262 \backslash C, 2.9795435424,-0.0014018617,-0$ $.0483373103 \backslash \mathrm{N}, 1.1839689627,1.5686078585,0.4653481228 \backslash \mathrm{H}, 1.7858044734,-0$ $.0504879634,1.7399090779 \backslash \mathrm{H}, 3.185298311,0.4914928878,-0.9894762078 \backslash \mathrm{H}, 3$. $5757181945,-0.8636544197,0.2093977343 \backslash \mathrm{H}, 1.1839125823,2.2682710755,1.21$ $34718158 \backslash \mathrm{H}, 1.5008869567,2.0292381006,-0.392417038 \backslash \mathrm{H}, 0.2114020713,1.252$ $1182431,0.3107796193 \backslash \mathrm{Br},-1.3489155904,-0.5326670106,-0.2734722874 \backslash \mathrm{H},-2$ $.6126406419,0.0997826218,-0.2578318969 \backslash \backslash$ Version $=x 86-$ Linux-G03RevB.03\S tate $=1-\mathrm{A} \backslash \mathrm{HF}=-2703.9231147 \backslash \mathrm{MP} 2=-2704.4979593 \backslash \mathrm{RMSD}=4.359 \mathrm{e}-09 \backslash \mathrm{RMSF}=1.631 \mathrm{e}$ $-06 \backslash \mathrm{Dipole}=1.8896471,2.3803842,0.6401836 \backslash \mathrm{PG}=\mathrm{C} 01 \quad[\mathrm{X}(\mathrm{C} 2 \mathrm{H} 7 \mathrm{Br} 1 \mathrm{~N} 1)] \backslash \backslash @$ 
$\mathrm{NH}_{2} \mathrm{CHCH}_{2} \cdots \mathrm{H}_{2} \mathrm{Br}^{+}(\mathrm{C} 3)$

$1 \backslash 1 \backslash G I N C-L C 58 \backslash F O p t \backslash R M P 2-F C \backslash 6-31+G(d, p) \backslash C 2 H 7 B r 1 N 1(1+) \backslash B U C 562 \backslash 08-N o v-200$ $4 \backslash 0 \backslash \backslash \#$ MP2 $/ 6-31+G(D, P) \quad O P T$ MAXDISK $=1342177280 \backslash \backslash \mathrm{CH} 2 \mathrm{CHNH} 2-\mathrm{H} 2 \mathrm{Br}+\backslash \backslash 1,1 \backslash \mathrm{C}$ $, 3.0373606087,2.2532574026,-0.3037443343 \backslash \mathrm{C}, 1.938709796,1.2734482892,-0$ $.2430091854 \backslash \mathrm{N}, 0.6985131447,1.5920182132,-0.0953706526 \backslash \mathrm{H}, 2.6909293432,3$ $.2792048078,-0.2119364488 \backslash \mathrm{H}, 3.7500059531,2.027835873,0.491893753 \backslash \mathrm{H}, 3.5$ $70550301,2.1223022889,-1.2472140343 \backslash \mathrm{H}, 2.1481860763,0.2116474264,-0.323$ $5595847 \backslash \mathrm{H}, 0.411524984,2.5636519019,-0.009084907 \backslash \mathrm{H},-0.0275559081,0.8698$ $301108,-0.0537556618 \backslash \mathrm{Br},-1.2948277122,-1.2030617974,0.1816831194 \backslash \mathrm{H},-1$. $9706852616,-1.271671144,-1.0571366089 \backslash \backslash$ Version=x86-Linux-G03RevB.03\St ate $=1-\mathrm{A} \backslash \mathrm{HF}=-2703.9513349 \backslash \mathrm{MP} 2=-2704.5194513 \backslash \mathrm{RMSD}=3.207 \mathrm{e}-09 \backslash \mathrm{RMSF}=1.637 \mathrm{e}-$ $05 \backslash \mathrm{Dipole}=2.1764786,2.5381341,-0.7065325 \backslash \mathrm{PG}=\mathrm{C} 01 \quad[\mathrm{X}(\mathrm{C} 2 \mathrm{H} 7 \mathrm{Br} 1 \mathrm{~N} 1)] \backslash \backslash @$

\section{$\mathrm{NH}_{2} \mathrm{CHCH}_{2} \cdots \bullet \mathrm{H}_{3} \mathrm{O}^{+}(\mathrm{N} 1)$}

$1 \backslash 1 \backslash$ GINC-SC160 $\mathrm{FOpt} \backslash \mathrm{RMP} 2-\mathrm{FC} \backslash 6-31+\mathrm{G}(\mathrm{d}, \mathrm{p}) \backslash \mathrm{C} 2 \mathrm{H} 8 \mathrm{~N} 101(1+) \backslash \mathrm{BUC} 562 \backslash 20-\mathrm{Oct}-200$ $4 \backslash 0 \backslash \backslash \#$ MP2 $/ 6-31+G(D, P)$ OPT MAXDISK $=134217728 \backslash \backslash \mathrm{CH} 2 \mathrm{CHNH} 2-\mathrm{H} 3 \mathrm{O}+\backslash \backslash 1,1 \backslash \mathrm{C}, 1$ $.0779742168,0.0061129261,0.4638821431 \backslash \mathrm{C}, 2.0445498322,-0.7114162662,-0$. $102253376 \backslash \mathrm{N}, 0.1292344341,0.7818388163,-0.3547038982 \backslash \mathrm{H}, 0.8883703282,0.0$ $819882162,1.5238051854 \backslash \mathrm{H}, 2.1940926402,-0.7554932109,-1.1729985548 \backslash \mathrm{H}, 2$. $7170279781,-1.2776742085,0.5237354984 \backslash \mathrm{H}, 0.162483381,1.7770508506,-0.11$ $75711789 \backslash \mathrm{H}, 0.3504558129,0.6970683433,-1.3496109295 \backslash \mathrm{H},-0.8542095389,0.4$ $474117521,-0.2103263484 \backslash 0,-2.4035710867,-0.1761585111,0.0960012815 \backslash \mathrm{H},-$ $3.2485902866,0.2878027695,0.0230637691 \backslash \mathrm{H},-2.6208469541,-1.0899380968,0$ $.3250469913 \backslash \backslash$ Version=DEC-AXP-OSF /1-G03RevB.03 \State $=1-A \backslash H F=-209.48713 \backslash$ MP $2=-210.1425557 \backslash \mathrm{RMSD}=4.288 \mathrm{e}-09 \backslash \mathrm{RMSF}=1.787 \mathrm{e}-06 \backslash \mathrm{Dipole}=-0.5518708,0.611$ $2329,-0.3836386 \backslash P G=C 01 \quad[X(C 2 H 8 N 101)] \backslash \backslash @$

\section{$\mathrm{NH}_{2} \mathrm{CHCH}_{2} \bullet \bullet \mathrm{H}_{3} \mathrm{O}^{+}(\mathrm{C} 3)$}

$1 \backslash 1 \backslash G I N C-L C 24 \backslash F O p t \backslash R M P 2-F C \backslash 6-31+G(d, p) \backslash C 2 H 8 N 101(1+) \backslash B U C 562 \backslash 02-N o v-2004$

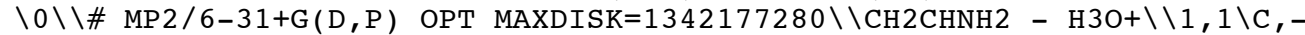
$0.2430674725,0.2 .3316586878 \backslash \mathrm{C},-0.3821063074,0 ., 0.8629267945 \backslash \mathrm{N}, 0.60984$ $30131,0.0 .0412391243 \backslash \mathrm{H}, 0.7952268424,0 ., 2.6530320877 \backslash \mathrm{H},-0.755950915,0$. $8756549096,2.7329536353 \backslash \mathrm{H},-0.755950915,-0.8756549096,2.7329536353 \backslash \mathrm{H},-1$ $.3683690711,0.0 .4105588263 \backslash \mathrm{H}, 1.5648679771,0 ., 0.3886556649 \backslash \mathrm{H}, 0.4519674$ $774,0 .,-0.9854262587 \backslash \mathrm{O},-0.0356562598,0 .,-2.6228581589 \backslash \mathrm{H},-0.0821998646$, $0.7717957111,-3.2030245419 \backslash \mathrm{H},-0.0821998646,-0.7717957111,-3.2030245419$ $\backslash \backslash$ Version $=x 86-$ Linux-G03RevB.03 $\backslash$ State $=1-A^{\prime} \backslash \mathrm{HF}=-209.5141196 \backslash \mathrm{MP} 2=-210.164$ $1031 \backslash \mathrm{RMSD}=6.845 \mathrm{e}-09 \backslash \mathrm{RMSF}=1.120 \mathrm{e}-05 \backslash \mathrm{Dipole}=0.1756331,0,,-0.1672647 \backslash \mathrm{PG}=\mathrm{C}$ $\mathrm{S}[\mathrm{SG}(\mathrm{C} 2 \mathrm{H} 4 \mathrm{~N} 1 \mathrm{O} 1), \mathrm{X}(\mathrm{H} 4)] \backslash \backslash @$

\section{$\mathrm{NH}_{2} \mathrm{CHCH}_{2} \cdots \bullet \mathrm{H}-\mathrm{NCH}^{+}(\mathrm{N1})$}

$1 \backslash 1 \backslash$ GINC-LC16 1 FOpt $\backslash$ RMP2-FC $\backslash 6-31+G(d, p) \backslash C 3 H 7 N 2(1+) \backslash$ BUC562 $\backslash 31-O c t-2004 \backslash 0$

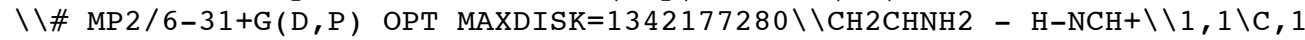
$.5429588166,-0.0943254039,0.4644759909 \backslash \mathrm{C}, 2.5019419328,-0.6853873762,-0$ $.2430366197 \backslash \mathrm{N}, 0.658438694,0.9118124426,-0.1486917516 \backslash \mathrm{H}, 1.3147446694,-0$ $.2890615316,1.5013009316 \backslash \mathrm{H}, 2.6900000769,-0.4573226313,-1.2838781868 \backslash \mathrm{H}$, $3.1266915387,-1.4267852611,0.2309786618 \backslash \mathrm{H}, 0.728395414,1.8140284514,0.3$ $302099274 \backslash \mathrm{H}, 0.9046201774,1.0662833382,-1.1294157493 \backslash \mathrm{H},-0.3426703001,0$. $6069115163,-0.1113552188 \backslash \mathrm{N},-2.0402657638,0.0072640824,-0.0139879952 \backslash \mathrm{C}$, $-3.1444338074,-0.3871235898,0.0327170637 \backslash \mathrm{H},-4.1517937397,-0.7465713376$ , $0.0759792528 \backslash \backslash$ Version $=x 86-$ Linux $-G 03 R e v B .03 \backslash$ State $=1-A \backslash H F=-226.3324087 \backslash$ MP $2=-227.0805691 \backslash \mathrm{RMSD}=6.942 \mathrm{e}-09 \backslash \mathrm{RMSF}=7.461 \mathrm{e}-06 \backslash \mathrm{Dipole}=-0.2126449,0.665$ $1696,-0.193757 \backslash \mathrm{PG}=\mathrm{C} 01 \quad[\mathrm{X}(\mathrm{C} 3 \mathrm{H} 7 \mathrm{~N} 2)] \backslash \backslash @$

\section{$\mathrm{NH}_{2} \mathrm{CHCH}_{2} \bullet \bullet \mathrm{H}_{-}-\mathrm{NCH}^{+}(\mathrm{C} 3)$}

$1 \backslash 1 \backslash G I N C-L C 24 \backslash F O p t \backslash R M P 2-F C \backslash 6-31+G(d, p) \backslash C 3 H 7 N 2(1+) \backslash B U C 562 \backslash 03-N o v-2004 \backslash 0$ $\backslash \backslash \#$ MP2 $/ 6-31+G(D, P)$ OPT SYMMETRY=LOOSE MAXDISK $=1342177280 \backslash \backslash \mathrm{CH} 2 \mathrm{CHNH} 2$ $\mathrm{H}-\mathrm{NCH}+\backslash \backslash 1,1 \backslash \mathrm{C},-0.1716534397,0.2 .8649321514 \backslash \mathrm{C},-0.3511713074,0 ., 1.39999$ $76845 \backslash \mathrm{N}, 0.6177114152,0.0 .5519652453 \backslash \mathrm{H},-0.6726342181,-0.8764239565,3.2$ $799892247 \backslash \mathrm{H},-0.6726342181,0.8764239565,3.2799892247 \backslash \mathrm{H}, 0.8755378736,0 .$, $3.1555665765 \backslash \mathrm{H},-1.3500178076,0 ., 0.9761252896 \backslash \mathrm{H}, 1.5824007161,0 ., 0.87193$ $89781 \backslash \mathrm{H}, 0.4376598958,0 .,-0.468905257 \backslash \mathrm{N}, 0.014886962,0 .,-2.2428877023 \backslash \mathrm{C}$, $-0.1868378762,0 .,-3.3990773138 \backslash \mathrm{H},-0.3705251428,0 .,-4.4533619705 \backslash \backslash$ Versi on $=x 86-$ Linux-G03RevB. 03 \State $=1-A^{\prime} \backslash \mathrm{HF}=-226.3587263 \backslash \mathrm{MP} 2=-227.1012058 \backslash \mathrm{RM}$ $\mathrm{SD}=4.533 e-09 \backslash \mathrm{RMSF}=9.383 e-05 \backslash \mathrm{Dipole}=0.0515137,0 ., 0.221191 \backslash \mathrm{PG}=\mathrm{CS} \quad[\mathrm{SG}(\mathrm{C} 3 \mathrm{H}$ $5 \mathrm{~N} 2), \mathrm{X}(\mathrm{H} 2) \mathrm{]} \backslash \backslash @$ 


\section{$\mathrm{NH}_{2} \mathrm{CHCH}_{2} \bullet \bullet \mathrm{H}-\mathrm{CNH}^{+}$(N1)}

$1 \backslash 1 \backslash$ GINC-LC80 $\backslash$ FOpt $\backslash$ RMP2-FC $\backslash 6-31+G(d, p) \backslash C 3 H 7 N 2(1+) \backslash B U C 562 \backslash 29-O c t-2004 \backslash 0$

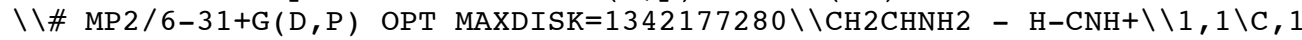
$.5985294705,0.1813745665,0.4591123158 \backslash \mathrm{C}, 2.635336909,-0.35300823,-0.180$ $7183812 \backslash \mathrm{N}, 0.6010795245,0.9940883515,-0.2562171352 \backslash \mathrm{H}, 1.3837491081,0.067$ $8316485,1.5108294509 \backslash \mathrm{H}, 2.8076448437,-0.2128522605,-1.239689516 \backslash \mathrm{H}, 3.342$ $4235752,-0.9547376343,0.3694017585 \backslash \mathrm{H}, 0.5467775265,1.9423828361,0.12578$ $70292 \backslash \mathrm{H}, 0.839143995,1.0762957899,-1.2476163489 \backslash \mathrm{H},-0.3587355223,0.56293$ $33706,-0.1867914035 \backslash \mathrm{C},-2.0574958377,-0.2800455591,-0.0064444656 \backslash \mathrm{N},-3.1$ $15452211,-0.7870849083,0.0929531288 \backslash \mathrm{H},-4.0186179717,-1.220802517,0.179$ $2302603 \backslash \backslash$ Version $=x 86-$ Linux $-G 03$ RevB. 03 $\backslash$ State $=1-A \backslash H F=-226.3157243 \backslash M P 2=-2$ $27.0529348 \backslash \mathrm{RMSD}=3.502 \mathrm{e}-09 \backslash \mathrm{RMSF}=1.533 \mathrm{e}-04 \backslash \mathrm{Dipole}=-0.3161215,0.6278314,-$ $0.2576735 \backslash \mathrm{PG}=\mathrm{C} 01[\mathrm{X}(\mathrm{C} 3 \mathrm{H} 7 \mathrm{~N} 2)] \backslash \backslash @$

\section{$\mathrm{NH}_{2} \mathrm{CHCH}_{2} \bullet \bullet \cdot \mathrm{H}-\mathrm{CNH}^{+}(\mathrm{C} 3)$}

$1 \backslash 1 \backslash$ GINC-LC2 $4 \backslash F O p t \backslash R M P 2-F C \backslash 6-31+G(d, p) \backslash C 3 H 7 N 2(1+) \backslash B U C 562 \backslash 03-N o v-2004 \backslash 0$ $\backslash \backslash \#$ MP2 $/ 6-31+G(D, P)$ OPT=LOOSE SYMMETRY=LOOSE MAXDISK $=1342177280 \backslash \backslash \mathrm{CH} 2 \mathrm{CH}$ $\mathrm{NH} 2-\mathrm{H}-\mathrm{CNH}+\backslash \backslash 1,1 \backslash \mathrm{C},-0.1833352898,0 ., 2.9270758519 \backslash \mathrm{C},-0.3449125673,0 ., 1$ $.4604112767 \backslash \mathrm{N}, 0.6330524409,0.0 .6235196518 \backslash \mathrm{H},-0.6899602505,0.875536337$ $9,3.3363782728 \backslash \mathrm{H},-0.6899602505,-0.8755363379,3.3363782728 \backslash \mathrm{H}, 0.85978407$ $63,0 ., 3.2322936067 \backslash \mathrm{H},-1.3389112703,0 \ldots, 1.0248289622 \backslash \mathrm{H}, 1.5940838438,0 \ldots, 0$ $.9548594336 \backslash \mathrm{H}, 0.4605913449,0 .,-0.4050349458 \backslash \mathrm{C}, 0.0261425294,0 .,-2.27867$ $93651 \backslash \mathrm{N},-0.1809223484,0 .,-3.4382643707 \backslash \mathrm{H},-0.3479061748,0 \ldots,-4.429337151$ $2 \backslash \backslash$ Version $=x 86-$ Linux $-G 03$ RevB. 03 $\backslash$ State $=1-A^{\prime} \backslash \mathrm{HF}=-226.3421185 \backslash M P 2=-227.07$ $32341 \backslash \mathrm{RMSD}=7.989 \mathrm{e}-09 \backslash \mathrm{RMSF}=6.705 \mathrm{e}-05 \backslash \mathrm{Dipole}=0.0571614,0,, 0.2087726 \backslash \mathrm{PG}=\mathrm{C}$ $\mathrm{S}[\mathrm{SG}(\mathrm{C} 3 \mathrm{H} 5 \mathrm{~N} 2), \mathrm{X}(\mathrm{H} 2)] \backslash \backslash @$

\section{$\mathrm{NH}_{2} \mathrm{CHCH}_{2} \cdots \bullet \mathrm{LiClH}^{+}$(N1)}

$1 \backslash 1 \backslash \mathrm{GINC}-\mathrm{LC} 53 \backslash \mathrm{FOpt} \backslash \mathrm{RMP} 2-\mathrm{FC} \backslash 6-31+\mathrm{G}(\mathrm{d}, \mathrm{p}) \backslash \mathrm{C} 2 \mathrm{H} 6 \mathrm{Cl} 1 \mathrm{Li} 1 \mathrm{~N} 1(1+) \backslash$ BUC562 $\backslash 30-$ OCt-

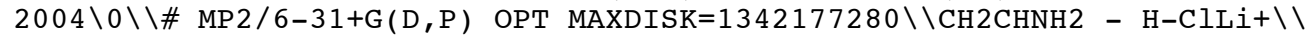
$1,1 \backslash C, 1.7996392333,-0.1847952978,0.4412574402 \backslash C, 2.8189392019,-0.613936$ $5487,-0.2980176832 \backslash \mathrm{N}, 0.9502174286,0.9272554595,-0.0087375633 \backslash \mathrm{H}, 1.48936$ $57592,-0.6013421785,1.3872626643 \backslash \mathrm{H}, 3.0827032214,-0.1685657046,-1.24804$ $55909 \backslash \mathrm{H}, 3.4150017147,-1.4419945145,0.053170387 \backslash \mathrm{H}, 0.9915466945,1.718844$ $749,0.6383844157 \backslash \mathrm{H}, 1.2363917222,1.262171967,-0.9310664374 \backslash \mathrm{H},-0.0566227$ $827,0.6254267049,-0.0673807919 \backslash \mathrm{Cl},-1.9123106094,-0.0993440871,-0.00310$ $74253 \backslash \mathrm{Li},-4.0040328604,-0.4680295602,-0.1925913386 \backslash \backslash$ Version $=x 86-\mathrm{Linux}-$ G03RevB.03 $\backslash$ State $=1-A \backslash H F=-600.4845334 \backslash M P 2=-601.0772602 \backslash \mathrm{RMSD}=2.965 \mathrm{e}-09 \backslash \mathrm{R}$ $\mathrm{MSF}=1.051 \mathrm{e}-05 \backslash \mathrm{Dipole}=-1.7382687,0.5568154,-0.3320781 \backslash \mathrm{PG}=\mathrm{C} 01 \quad[\mathrm{X}(\mathrm{C} 2 \mathrm{H} 6 \mathrm{C} 11$ Li1N1) ] \\@

\section{$\mathrm{NH}_{2} \mathrm{CHCH}_{2} \cdots \mathrm{LiClH}^{+}(\mathrm{C} 3)$}

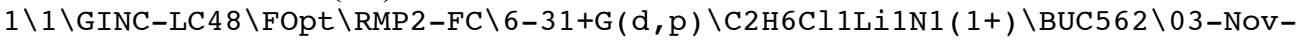
$2004 \backslash 0 \backslash \backslash \#$ MP2 $/ 6-31+G(D, P)$ OPT SYMMETRY=LOOSE MAXDISK $=1342177280 \backslash \backslash \mathrm{CH} 2 \mathrm{CH}$ $\mathrm{NH} 2-\mathrm{H}-\mathrm{ClLi}+\backslash \backslash 1,1 \backslash \mathrm{C},-0.4152811122,0 ., 3.0406932416 \backslash \mathrm{C},-0.317479703,0 ., 1$ $.5678924253 \backslash \mathrm{N}, 0.7901132367,0 ., 0.9137911085 \backslash \mathrm{H}, 0.5582775801,0 ., 3.5240645$ $64 \backslash \mathrm{H},-0.9846238042,0.8760144276,3.3548316578 \backslash \mathrm{H},-0.9846238042,-0.876014$ $4276,3.3548316578 \backslash \mathrm{H},-1.213728177,0.0 .9559676672 \backslash \mathrm{H}, 1.6851883201,0 ., 1.3$ $934512305 \backslash \mathrm{H}, 0.7579592246,0 .,-0.1240295376 \backslash \mathrm{Cl},-0.0667129828,0,-2.00638$ $56805 \backslash \mathrm{Li}, 0.060481201,0 .,-4.1328708105 \backslash \backslash$ Version $=x 86-\mathrm{Linux}-\mathrm{G} 03 \mathrm{RevB} .03 \backslash \mathrm{St}$ ate $=1-A^{\prime} \backslash \mathrm{HF}=-600.5104915 \backslash \mathrm{MP} 2=-601.0971559 \backslash \mathrm{RMSD}=3.463 \mathrm{e}-09 \backslash \mathrm{RMSF}=5.290 \mathrm{e}-0$ $6 \backslash$ Dipole $=0.5388059,0 .,-1.2794489 \backslash P G=C S \quad[S G(C 2 H 4 C l 1 L i 1 N 1), x(H 2)] \backslash \backslash @$

\section{$\mathrm{NH}_{2} \mathrm{CHCH}_{2} \cdots \bullet \mathrm{NH}_{4}^{+}$(N1)}

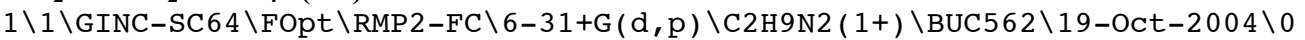

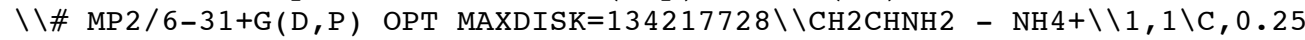
$53942226,-0.878406319,0.7409713145 \backslash C, 1.5922514934,-0.8960897773,0.7464$ $48214 \backslash \mathrm{N},-0.5241085239,0.3326822657,0.8193350961 \backslash \mathrm{H},-0.3344662879,-1.777$ $0878523,0.6156289479 \backslash \mathrm{H}, 2.1819771189,-0.0003106043,0.893728097 \backslash \mathrm{H}, 2.1171$ $232986,-1.831339401,0.6300196104 \backslash \mathrm{H},-1.3658186806,0.1856633977,1.373376$ $2673 \backslash \mathrm{H}, 0.0079331708,1.0640317038,1.2898952552 \backslash \mathrm{H},-0.811765822,0.7573284$ $563,-0.6866844044 \backslash \mathrm{N},-0.9122163049,0.9722820663,-1.7732349421 \backslash \mathrm{H},-0.8814$ $945654,1.9759502298,-1.9490284434 \backslash \mathrm{H},-0.1494251389,0.528072533,-2.28380$ $31973 \backslash \mathrm{H},-1.7956635874,0.6099177906,-2.1303503821 \backslash \backslash$ Version=DEC-AXP-OSF / $1-\mathrm{G} 03$ RevB.03 $\backslash$ State $=1-\mathrm{A} \backslash \mathrm{HF}=-189.6576272 \backslash \mathrm{MP} 2=-190.3084613 \backslash \mathrm{RMSD}=6.732 \mathrm{e}-09$ $\backslash \mathrm{RMSF}=1.206 \mathrm{e}-05 \backslash \mathrm{Dipole}=-1.3042225,1.3279038,-1.7305993 \backslash \mathrm{PG}=\mathrm{C} 01 \quad[\mathrm{X}(\mathrm{C} 2 \mathrm{H} 9 \mathrm{~N}$ 2) $] \backslash \backslash @$ 


\section{$\mathrm{NH}_{2} \mathrm{CHCH}_{2} \cdots \mathrm{NH}_{3} \mathrm{Me}^{+}(\mathrm{N} 1)$}

$1 \backslash 1 \backslash$ GINC-LC1 $12 \backslash F O p t \backslash R M P 2-F C \backslash 6-31+G(d, p) \backslash C 3 H 11 N 2(1+) \backslash B U C 562 \backslash 03-N o v-2004$ $\backslash 0 \backslash \backslash \#$ MP2 $/ 6-31+G(D, P)$ OPT MAXDISK $=1342177280 \backslash \backslash \mathrm{CH} 2 \mathrm{CHNH} 2-\mathrm{NH} 3 \mathrm{Me}+\backslash \backslash 1,1 \backslash \mathrm{C}$ $, 0.6405032276,-0.5547570537,-1.3212482732 \backslash \mathrm{C}, 1.9254048342,-0.2141591143$ $,-1.1600484522 \backslash \mathrm{N},-0.0720225508,-1.4177729023,-0.4226422205 \backslash \mathrm{H}, 0.0352077$ $586,-0.1394329424,-2.1170996045 \backslash \mathrm{H}, 2.5438198842,-0.6449524554,-0.382494$ $5685 \backslash \mathrm{H}, 2.3889753295,0.4752521569,-1.8481495569 \backslash \mathrm{H},-0.8249483079,-1.9074$ $424782,-0.8985282833 \backslash \mathrm{H}, 0.5450048389,-2.1294814805,-0.0355509959 \backslash \mathrm{H},-0.5$ $077755073,-0.3317884182,0.8296841236 \backslash \mathrm{N},-0.6695316992,0.4717360355,1.52$ $3329203 \backslash \mathrm{C},-1.4368046768,1.5831041053,0.8791111299 \backslash \mathrm{H},-1.1643667086,0.10$ $51805201,2.3374546218 \backslash \mathrm{H}, 0.2425862268,0.7983132975,1.845101912 \backslash \mathrm{H},-2.390$ $3207538,1.1887788254,0.5446244063 \backslash \mathrm{H},-1.5914137559,2.3794699854,1.59922$ $49115 \backslash \mathrm{H},-0.8605095653,1.943233433,0.0340377298 \backslash \backslash$ Version $=x 86-L$ inux-G03R evB.03 $\backslash$ State $=1-A \backslash H F=-228.6966872 \backslash M P 2=-229.4882904 \backslash \mathrm{RMSD}=6.466 \mathrm{e}-09 \backslash \mathrm{RMSF}=$ $6.721 \mathrm{e}-06 \backslash \mathrm{Dipole}=-0.9808804,0.2056227,1.6240801 \backslash \mathrm{PG}=\mathrm{C} 01 \quad[\mathrm{X}(\mathrm{C} 3 \mathrm{H} 11 \mathrm{~N} 2)] \backslash \backslash @$

\section{$\mathrm{NH}_{2} \mathrm{CHCH}_{2} \cdots \mathrm{LiCNH}^{+}$(N1)}

$1 \backslash 1 \backslash$ GINC-SC2 $\backslash F O p t \backslash R M P 2-F C \backslash 6-31+G(d, p) \backslash C 3 H 6 L i 1 N 2(1+) \backslash B U C 562 \backslash 02-N o v-2004$

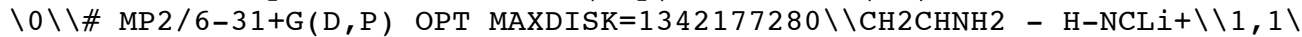
$C, 1.742801567,-0.1195908381,0.4580510128 \backslash C, 2.655475366,-0.8160721291,-$ $0.216364778 \backslash \mathrm{N}, 0.9068932547,0.8878284042,-0.1951636038 \backslash \mathrm{H}, 1.5170056767,-$ $0.2511678733,1.5056542582 \backslash \mathrm{H}, 2.8469308741,-0.6595083684,-1.269603223 \backslash \mathrm{H}$, $3.234010953,-1.5699169874,0.2946538627 \backslash \mathrm{H}, 1.0170418432,1.8049831992,0.2$ $426131705 \backslash \mathrm{H}, 1.1576871314,0.9871481646,-1.180289072 \backslash \mathrm{H},-0.1557850743,0.6$ $125431844,-0.1370434428 \backslash \mathrm{N},-1.6431271203,0.189521071,-0.0312527157 \backslash \mathrm{C},-2$ $.7822314626,-0.1350213808,0.0384538658 \backslash \mathrm{Li},-4.7198423892,-0.6804738526$, $0.149362693 \backslash \backslash$ Version=DEC-AXP-OSF $/ 1-$ G03RevB . 03 $\backslash$ State $=1-A \backslash H F=-233.265551$ $7 \backslash M P 2=-234.0149289 \backslash \mathrm{RMSD}=9.517 \mathrm{e}-09 \backslash \mathrm{RMSF}=2.171 \mathrm{e}-05 \backslash \mathrm{Dipole}=-3.0154642,-0$. $0513177,-0.0517621 \backslash P G=C 01 \quad[X(C 3 H 6 L i 1 N 2)] \backslash \backslash @$

\section{$\mathrm{NH}_{2} \mathrm{CHCH}_{2} \bullet \bullet \mathrm{LiCNH}^{+}(\mathrm{C3})$}

$1 \backslash 1 \backslash$ GINC-LC1 42\FOpt \RMP2-FC \6-31+G (d,p) \C3H6Li1N2 (1+) \BUC562\02-Nov-20

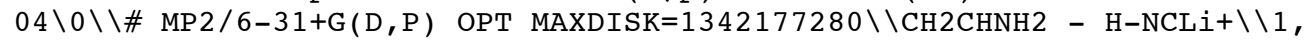
$1 \backslash \mathrm{C},-1.6224590628,0.5248357699,0.4333172106 \backslash \mathrm{C},-0.9642429057,1.53330189$ $53,-0.1945547542 \backslash \mathrm{N},-2.0080838354,-0.6606079165,-0.1425186372 \backslash \mathrm{H},-1.7841$ $025478,0.572763503,1.5046079494 \backslash \mathrm{H},-0.8681479885,1.5582247201,-1.274063$ $7717 \backslash \mathrm{H},-0.7464825038,2.4375896281,0.352973874 \backslash \mathrm{H}, 0.52891554,0.422793697$ $8,0.0277803349 \backslash \mathrm{N}, 1.4252474106,-0.1424530204,0.0030684765 \backslash \mathrm{H},-2.65248693$ $66,-1.2367820399,0.3754532206 \backslash \mathrm{H},-2.1676299214,-0.667951708,-1.13977198$ $9 \backslash \mathrm{C}, 2.4352799779,-0.7439817087,-0.020838934 \backslash \mathrm{Li}, 4.2261070916,-1.7833823$ $271,-0.0594565426 \backslash \backslash$ Version $=x 86-$ Linux-G03RevB.03 $\backslash$ State $=1-A \backslash H F=-233.2471$ $236 \backslash M P 2=-233.9960816 \backslash \mathrm{RMSD}=5.341 \mathrm{e}-09 \backslash \mathrm{RMSF}=3.508 \mathrm{e}-05 \backslash \mathrm{Dipole}=4.5376378,-2$ $.4217019,-0.0596872 \backslash \mathrm{PG}=\mathrm{C} 01 \quad[\mathrm{X}(\mathrm{C} 3 \mathrm{H} 6 \mathrm{Li} 1 \mathrm{~N} 2)] \backslash \backslash @$

\section{$\mathrm{NH}_{2} \mathrm{CHCH}_{2} \cdots \mathrm{NH}_{2} \mathrm{Me}_{2}^{+}(\mathrm{N} 1)$}

$1 \backslash 1 \backslash G I N C-L C 74 \backslash F O p t \backslash R M P 2-F C \backslash 6-31+G(d, p) \backslash C 4$ H13N2 $(1+) \backslash B U C 562 \backslash 03-N o v-2004 \backslash$ $0 \backslash \backslash \#$ MP2 $/ 6-31+G(D, P)$ OPT MAXDISK=1342177280 \CH2CHNH2 - NH2(Me) $2+\backslash \backslash 1,1$ $\backslash C, 0.7481843959,-0.7403954338,-1.5910551174 \backslash C, 1.9717967737,-0.91983921$ $22,-1.0766743233 \backslash \mathrm{N},-0.4416306232,-1.3307526509,-1.0532377261 \backslash \mathrm{H}, 0.57529$ $6399,-0.0733189856,-2.4264877685 \backslash \mathrm{H}, 2.1587252341,-1.604642611,-0.258977$ $4609 \backslash \mathrm{H}, 2.8174398767,-0.4088577902,-1.509839755 \backslash \mathrm{H},-1.1302154678,-1.5015$ $161335,-1.7810894469 \backslash \mathrm{H},-0.2394084795,-2.2296133947,-0.6202673416 \backslash \mathrm{H},-0$. $912438408,-0.1644139125,0.1937584645 \backslash \mathrm{N},-1.0272685188,0.5853662283,0.93$ $69779333 \backslash \mathrm{H},-1.9952916209,0.5326257292,1.2609120439 \backslash \mathrm{C},-0.1135934662,0.2$ $876840782,2.0791979351 \backslash \mathrm{C},-0.7897949043,1.9245453753,0.3216978866 \backslash \mathrm{H}, 0.9$ $070058262,0.3293323967,1.7115744627 \backslash \mathrm{H},-1.48317172,2.0586763423,-0.5031$ $712335 \backslash \mathrm{H},-0.2647508626,1.0289554998,2.8584246909 \backslash \mathrm{H},-0.3378521993,-0.70$ $53824344,2.4569020078 \backslash \mathrm{H},-0.946029661,2.6936562636,1.0725359033 \backslash \mathrm{H}, 0.233$ $4282818,1.9502351443,-0.0394543037 \backslash \backslash$ Version=x86-Linux-G03RevB.03\State $=1-\mathrm{A} \backslash \mathrm{HF}=-267.7345792 \backslash \mathrm{MP} 2=-268.6709773 \backslash \mathrm{RMSD}=6.586 \mathrm{e}-09 \backslash \mathrm{RMSF}=1.348 \mathrm{e}-05 \backslash \mathrm{Di}$ pole $=-1.3056872,0.3706098,0.8558174 \backslash \mathrm{PG}=\mathrm{C} 01[\mathrm{X}(\mathrm{C} 4 \mathrm{H} 13 \mathrm{~N} 2)] \backslash \backslash @$ 
$\mathrm{NH}_{2} \mathrm{CHCH}_{2} \cdots \mathrm{NH}_{2} \mathrm{Me}_{2}^{+}(\mathrm{C} 3)$

$1 \backslash 1 \backslash$ GINC-LC5 $1 \backslash F O p t \backslash R M P 2-F C \backslash 6-31+G(d, p) \backslash C 4 H 13 N 2(1+) \backslash B U C 562 \backslash 03-N o v-2004 \backslash$ $0 \backslash \backslash \#$ MP2 $/ 6-31+G(D, P)$ OPT MAXDISK=1342177280 \\CH2CHNH2 - NH2(Me) $2+\backslash \backslash 1,1$ $\backslash C,-0.6994117594,-0.9006645495,-1.4933791428 \backslash C, 0.5910018116,-1.2462286$ $999,-1.6957564353 \backslash \mathrm{N},-1.4450524253,-1.2200624609,-0.3544901777 \backslash \mathrm{H},-1.209$ $1962743,-0.2537851746,-2.1989471204 \backslash \mathrm{H}, 1.1027253428,-1.9465719894,-1.04$ $5088389 \backslash \mathrm{H}, 1.0893986637,-0.9438118637,-2.6038787685 \backslash \mathrm{H}, 0.6633801304,0.26$ $97129863,-0.167728588 \backslash \mathrm{N}, 0.8282439138,0.9318013848,0.6159685919 \backslash \mathrm{H},-2.44$ $62421731,-1.1641026398,-0.4882704894 \backslash \mathrm{H},-1.2009322475,-2.107904574,0.06$ $94800557 \backslash \mathrm{H}, 1.7388745251,1.3599716954,0.4331245902 \backslash \mathrm{C},-0.2282003812,1.98$ $84874877,0.5895837408 \backslash \mathrm{C}, 0.8867760338,0.1769203835,1.904091321 \backslash \mathrm{H}, 1.6665$ $88092,-0.5752543582,1.8327685199 \backslash \mathrm{H},-1.1837897904,1.5017365618,0.753202$ $08 \backslash \mathrm{H}, 1.1079334263,0.8739174053,2.7067743146 \backslash \mathrm{H},-0.081026425,-0.28912738$ $28,2.0551479501 \backslash \mathrm{H},-0.02134726,2.7071580015,1.3767733683 \backslash \mathrm{H},-0.209700658$ $4,2.4748011329,-0.3809433254 \backslash \backslash$ Version $=x 86-$ Linux-G03RevB.03 $\backslash$ State $=1-A \backslash H$ $\mathrm{F}=-267.7303871 \backslash \mathrm{MP} 2=-268.666213 \backslash \mathrm{RMSD}=7.443 e-09 \backslash \mathrm{RMSF}=3.858 \mathrm{e}-05 \backslash \mathrm{Dipole}=0$. $4563344,0.9041992,0.8556375 \backslash P G=C 01 \quad[X(C 4 H 13 N 2)] \backslash \backslash @$

\section{$\mathrm{NH}_{2} \mathrm{CHCH}_{2} \cdots \mathrm{NHMe}_{3}^{+}(\mathrm{N} 1)$}

$1 \backslash 1 \backslash G I N C-L C 124 \backslash F O p t \backslash R M P 2-F C \backslash 6-31+G(d, p) \backslash C 5 H 15 N 2(1+) \backslash B U C 562 \backslash 29-O c t-2004$ $\backslash 0 \backslash \backslash \#$ MP2 /6-31+G(D,P) OPT MAXDISK=1342177280 \CH2CHNH2 - NH(Me) $3+\backslash \backslash 1,1$ $\backslash C, 2.1935890094,0.2961179306,0.392914 \backslash C, 2.6881399741,-0.8174770851,-0$. $163913 \backslash \mathrm{N}, 1.3795170394,1.2465219563,-0.303106 \backslash \mathrm{H}, 2.3329210163,0.51520592$ $62,1.444388 \backslash \mathrm{H}, 2.5757099673,-1.0328640815,-1.219158 \backslash \mathrm{H}, 3.2600449522,-1.5$ $097361032,0.434087 \backslash \mathrm{H}, 1.5037000691,2.1834339524,0.070288 \backslash \mathrm{H}, 1.6246130406$ $, 1.2845529486,-1.29027 \backslash \mathrm{H},-0.2488489835,0.5210010079,-0.122888 \backslash \mathrm{N},-1.146$ $2250008,-0.0240659637,-0.00168 \backslash C,-2.2772439708,0.9219350721,-0.225036 \backslash$ $C,-1.1425890355,-1.1203379638,-1.013026 \backslash C,-1.154844018,-0.5694989635,1$ $.386654 \backslash \mathrm{H},-2.2019029581,1.3252800697,-1.230937 \backslash \mathrm{H},-0.2869650558,-1.7619$ $189909,-0.823406 \backslash \mathrm{H},-1.0946009918,0.2585870346,2.087616 \backslash \mathrm{H},-3.2148279878$ $, 0.3841981017,-0.106207 \backslash \mathrm{H},-2.2112639454,1.72419407,0.504494 \backslash \mathrm{H},-2.06700$ $00534,-1.6860299346,-0.922181 \backslash \mathrm{H},-1.0711790216,-0.6810489661,-2.004305 \backslash$ $\mathrm{H},-2.0788710355,-1.1220259342,1.541156 \backslash \mathrm{H},-0.2968890387,-1.2244519906,1$ $.50527 \backslash \backslash$ Version $=x 86-$ Linux-G03RevB.03 $\backslash$ State $=1-A \backslash H F=-306.7710254 \backslash M P 2=-30$ $7.8554024 \backslash \mathrm{RMSD}=5.376 \mathrm{e}-09 \backslash \mathrm{RMSF}=1.411 \mathrm{e}-05 \backslash \mathrm{Dipole}=-1.0512317,0.3782627,-0$ $.1056115 \backslash P G=C 01[X(C 5 H 15 N 2)] \backslash \backslash @$

\section{$\mathrm{NH}_{2} \mathrm{CHCH}_{2} \cdots \mathrm{NHMe}_{3}^{+}(\mathrm{C} 3)$}

$1 \backslash 1 \backslash$ GINC-LC61 \FOpt \RMP2-FC $\backslash 6-31+\mathrm{G}(\mathrm{d}, \mathrm{p}) \backslash \mathrm{C} 5 \mathrm{H} 15 \mathrm{~N} 2(1+) \backslash \mathrm{BUC} 562 \backslash 29-$ Oct $-2004 \backslash$ $0 \backslash \backslash \#$ MP2 $/ 6-31+G(D, P)$ OPT MAXDISK=1342177280 \\CH2CHNH2 - NH(Me) $3+\backslash \backslash 1,1 \backslash$ C $, 2.0933409671,0.2655132595,-0.42087 \backslash C, 1.7347688251,1.410965215,0.2019$ $1 \backslash \mathrm{N}, 2.1329931233,-0.9944617356,0.176352 \backslash \mathrm{H}, 2.2901399673,0.2636492839,-1$ $.487494 \backslash \mathrm{H}, 1.6028668194,1.4572821987,1.277302 \backslash \mathrm{H}, 1.73217471,2.3393842147$ $,-0.348508 \backslash \mathrm{H},-0.1160260496,0.3998779856,-0.029255 \backslash \mathrm{N},-1.075625,0.000031$ $8667,-0.010206 \backslash \mathrm{H}, 2.6981542073,-1.6725586655,-0.317005 \backslash \mathrm{H}, 2.3662211227,-$ $0.9897647067,1.162392 \backslash C,-2.0263261421,1.1461087488,-0.122596 \backslash C,-1.1903$ $508851,-0.9271641476,-1.174823 \backslash C,-1.2373459101,-0.7254141534,1.284371 \backslash$ $\mathrm{H},-1.0834659973,-0.0221541343,2.098145 \backslash \mathrm{H},-1.8328302067,1.6676037728,-1$ $.055476 \backslash \mathrm{H},-0.4499067877,-1.7123890558,-1.058772 \backslash \mathrm{H},-2.2440068595,-1.133$ $7932782,1.329445 \backslash \mathrm{H},-0.4989418116,-1.5200600618,1.322696 \backslash \mathrm{H},-3.041453093$ $9,0.757588623,-0.110448 \backslash \mathrm{H},-1.8677532248,1.8136797685,0.719424 \backslash \mathrm{H},-2.194$ $6498335,-1.343485272,-1.187956 \backslash \mathrm{H},-1.0066239549,-0.3639021248,-2.085462$ $\backslash \backslash$ Version $=x 86-L$ inux-G03RevB.03 $\backslash$ State $=1-\mathrm{A} \backslash \mathrm{HF}=-306.7671219 \backslash \mathrm{MP} 2=-307.8514$ $414 \backslash \mathrm{RMSD}=5.336 \mathrm{e}-09 \backslash \mathrm{RMSF}=1.623 \mathrm{e}-05 \backslash \mathrm{Dipole}=-0.8480213,-0.3637806,-0.0038$ $156 \backslash P G=C 01 \quad[X(C 5 H 15 N 2)] \backslash \backslash @$

\section{$\mathrm{NH}_{2} \mathrm{CHCH}_{2} \bullet \bullet \mathrm{NaCNH}^{+}(\mathrm{N} 1)$}

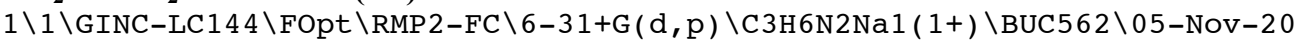

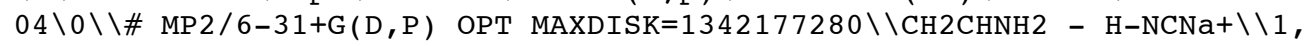
$1 \backslash C, 2.5733929991,0.0170672963,0.4354118165 \backslash C, 3.4722810933,-0.698663632$ $5,-0.2382379768 \backslash \mathrm{N}, 1.7386408852,1.0208852727,-0.2179700182 \backslash \mathrm{H}, 2.35721622$ $37,-0.1039199509,1.4864859091 \backslash \mathrm{H}, 3.6561910536,-0.5553897932,-1.29468419$ $15 \backslash \mathrm{H}, 4.0460042761,-1.4541670964,0.2756547996 \backslash \mathrm{H}, 1.8467737238,1.93752202$ $17,0.2202668577 \backslash \mathrm{H}, 1.9920530577,1.120768714,-1.201958712 \backslash \mathrm{H}, 0.6525868615$ $, 0.7365425025,-0.1599206199 \backslash \mathrm{N},-0.7741848716,0.3295120879,-0.061683334 \backslash$ $\mathrm{C},-1.9142297158,0.0028768252,0.0017255015 \backslash \mathrm{Na},-4.190062141,-0.641983714$ $2,0.1307575796 \backslash \backslash$ Version $=x 86-$ Linux - G03RevB. 03 $\backslash$ State $=1-A \backslash H F=-387.6646391$ $\backslash M P 2=-388.4142832 \backslash \mathrm{RMSD}=8.537 \mathrm{e}-09 \backslash \mathrm{RMSF}=3.031 \mathrm{e}-05 \backslash \mathrm{Dipole}=-2.4119089,-0.0$ $419877,-0.051365 \backslash P G=C 01[X(C 3 H 6 N 2 N a 1)] \backslash \backslash @$ 


\section{$\mathrm{NH}_{2} \mathrm{CHCH}_{2} \cdots \mathrm{NaCNH}^{+}(\mathrm{C} 3)$}

$1 \backslash 1 \backslash G I N C-L C 32 \backslash F O p t \backslash R M P 2-F C \backslash 6-31+G(d, p) \backslash C 3 H 6 N 2 N a 1(1+) \backslash B U C 562 \backslash 05-N o v-200$ $4 \backslash 0 \backslash \backslash \#$ MP2 $/ 6-31+G(D, P)$ OPT MAXDISK $=1342177280 \backslash \backslash \mathrm{CH} 2 \mathrm{CHNH} 2-\mathrm{H}-\mathrm{NCNa}+\backslash \backslash 1,1$ $\backslash C,-2.3880421082,0.8855681405,0.4503732742 \backslash C,-1.7634767799,1.909483344$ $1,-0.1809649588 \backslash \mathrm{N},-2.7323093644,-0.3206226017,-0.1225163746 \backslash \mathrm{H},-2.56013$ $27998,0.9355448431,1.5197959433 \backslash \mathrm{H},-1.6436692799,1.9234313612,-1.258063$ $1168 \backslash \mathrm{H},-1.5563059017,2.817868102,0.3633229479 \backslash \mathrm{H},-0.2123945269,0.735128$ $8201,0.0464235867 \backslash \mathrm{N}, 0.6687464793,0.1750886129,0.0150009979 \backslash \mathrm{H},-3.386456$ $0913,-0.8925294391,0.3885361053 \backslash \mathrm{H},-2.8964834099,-0.3251564664,-1.11934$ $17512 \backslash \mathrm{C}, 1.6764221613,-0.4336552506,-0.0146134065 \backslash \mathrm{Na}, 3.7773602333,-1.66$ $76297004,-0.0651668665 \backslash \backslash$ Version $=x 86-$ Linux-G03RevB.03 $\backslash$ State $=1-A \backslash H F=-387$ $.6542555 \backslash M P 2=-388.4011575 \backslash \mathrm{RMSD}=7.768 \mathrm{e}-09 \backslash \mathrm{RMSF}=2.788 \mathrm{e}-05 \backslash \mathrm{Dipole}=3.99045$ $81,-2.2599254,-0.0722333 \backslash \mathrm{PG}=\mathrm{C} 01[\mathrm{X}(\mathrm{C} 3 \mathrm{H} 6 \mathrm{~N} 2 \mathrm{Na} 1)] \backslash \backslash @$

\section{$\mathrm{NH}_{2} \mathrm{CHCH}_{2} \cdots \mathrm{LiNCH}^{+}$(N1)}

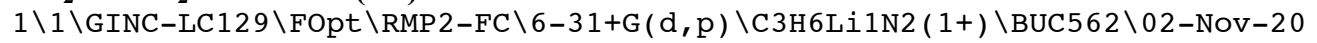

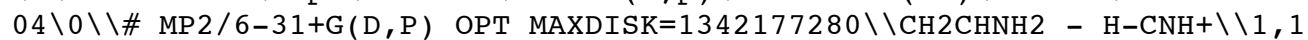
$\backslash C, 1.7341868006,0.4523971263,0.4649373872 \backslash \mathrm{C}, 2.2750540469,-0.5951444994$ $,-0.1722883626 \backslash \mathrm{N}, 0.9307222962,1.4447099419,-0.1680735968 \backslash \mathrm{H}, 1.812565053$ $2,0.5619849908,1.539255975 \backslash \mathrm{H}, 2.2123386787,-0.7094203588,-1.2468731592 \backslash$ $\mathrm{H}, 2.8229834133,-1.3390589443,0.3841616354 \backslash \mathrm{H}, 1.0004842625,2.3444136507$, $0.2962914545 \backslash \mathrm{H}, 1.1936788402,1.5757310247,-1.1413859025 \backslash \mathrm{H},-0.6786877826$ $, 0.5755519121,-0.1173165092 \backslash C,-1.5595482576,-0.1057507016,-0.067115538$ $\backslash N,-2.4965026078,-0.8115752767,-0.016431967 \backslash \mathrm{Li},-4.0336852747,-1.983385$ $4946,0.0747348441 \backslash \backslash$ Version=x86-Linux-G03RevB.03 $\backslash$ State $=1-A \backslash H F=-233.2724$ $631 \backslash M P 2=-234.0223492 \backslash \mathrm{RMSD}=8.858 \mathrm{e}-09 \backslash \mathrm{RMSF}=5.843 \mathrm{e}-06 \backslash \mathrm{Dipole}=-4.8446301$, $1.8399797,0.0047979 \backslash \mathrm{PG}=\mathrm{C} 01 \quad[\mathrm{X}(\mathrm{C} 3 \mathrm{H} 6 \mathrm{Li} 1 \mathrm{~N} 2)] \backslash \backslash @$

\section{$\mathrm{NH}_{2} \mathrm{CHCH}_{2} \cdots \mathrm{LiNCH}^{+}(\mathrm{C3})$}

$1 \backslash 1 \backslash$ GINC-LC $41 \backslash F O p t \backslash R M P 2-F C \backslash 6-31+G(d, p) \backslash C 3 H 6 L i 1 N 2(1+) \backslash B U C 562 \backslash 02-N o v-200$

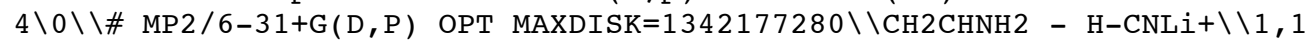
$\backslash C,-1.6810314313,0.6542840428,0.4473560305 \backslash C,-1.2438630485,1.758082386$ $7,-0.1928137153 \backslash \mathrm{N},-1.7291282162,-0.6262374077,-0.1114374398 \backslash \mathrm{H},-1.92889$ $69544,0.693731814,1.5017334306 \backslash \mathrm{H},-1.0237277324,1.7551426797,-1.2539451$ $016 \backslash \mathrm{H},-1.2116586714,2.7026227259,0.3271665921 \backslash \mathrm{H}, 0.5330037988,0.3583542$ $712,0.0391065424 \backslash \mathrm{C}, 1.4258625885,-0.2583587892,-0.0061206549 \backslash \mathrm{H},-2.33043$ $99373,-1.2747956833,0.3769212552 \backslash \mathrm{H},-1.9127827862,-0.6482228583,-1.1069$ $326949 \backslash \mathrm{N}, 2.4078294207,-0.8981722274,-0.0461016856 \backslash \mathrm{Li}, 4.0392617331,-1.9$ $466704483,-0.0906020359 \backslash \backslash$ Version $=x 86-$ Linux-G03RevB.03 $\backslash$ State $=1-A \backslash H F=-23$ $3.2678734 \backslash \mathrm{MP} 2=-234.0180854 \backslash \mathrm{RMSD}=5.504 \mathrm{e}-09 \backslash \mathrm{RMSF}=4.675 \mathrm{e}-05 \backslash \mathrm{Dipole}=4.7147$ $63,-2.6657823,-0.1303625 \backslash P G=C 01 \quad[X(C 3 H 6 L i 1 N 2)] \backslash \backslash @$

\section{$\mathrm{NH}_{2} \mathrm{CHCH}_{2} \cdots \mathrm{HBr}(\mathrm{N} 1)$}

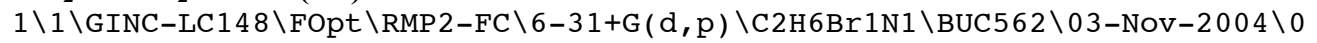

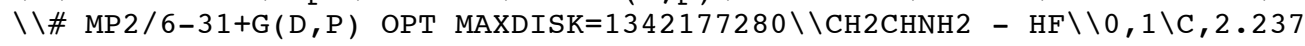
$5630548,0.5770546555,0.4335510298 \backslash C, 3.0985637235,-0.2223128734,-0.2110$ $116898 \backslash \mathrm{N}, 1.2285776415,1.3461345303,-0.1972769425 \backslash \mathrm{H}, 2.2016318443,0.6116$ $785794,1.5150535625 \backslash \mathrm{H}, 3.1295454138,-0.2778604092,-1.2911300061 \backslash \mathrm{H}, 3.777$ $3575775,-0.8461721016,0.3485670369 \backslash \mathrm{H}, 1.0746395967,2.2417144008,0.25260$ $89503 \backslash \mathrm{H}, 1.4126611632,1.4929363702,-1.1847490045 \backslash \mathrm{H},-0.2895027047,0.3951$ $956113,-0.0943587179 \backslash \mathrm{Br},-1.4835182015,-0.4333967102,0.0142774496 \backslash \backslash$ Vers ion $=x 86-L$ inux-G03RevB .03 \State $=1-A \backslash H F=-2703.5718803 \backslash M P 2=-2704.1544457 \backslash$ $\mathrm{RMSD}=5.972 \mathrm{e}-09 \backslash \mathrm{RMSF}=1.081 \mathrm{e}-05 \backslash \mathrm{Dipole}=1.2127083,1.0314338,-0.1112836 \backslash \mathrm{PG}$ $=\mathrm{C} 01[\mathrm{X}(\mathrm{C} 2 \mathrm{H} 6 \mathrm{Br} 1 \mathrm{~N} 1)] \backslash \backslash @$

\section{$\mathrm{NH}_{2} \mathrm{CHCH}_{2} \cdots \cdot \mathrm{HBr}(\mathrm{C} 3)$}

$1 \backslash 1 \backslash G I N C-L C 6 \backslash F O p t \backslash R M P 2-F C \backslash 6-31+G(d, p) \backslash C 2 H 6 B r 1 N 1 \backslash B U C 562 \backslash 03-N o v-2004 \backslash 0 \backslash \backslash$

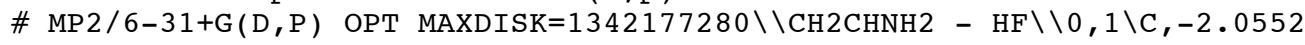
$276234,0.7528156321,0.4642200249 \backslash C,-1.5358812644,1.8304068441,-0.16235$ $26087 \backslash \mathrm{N},-2.3929706168,-0.4513136465,-0.1343180567 \backslash \mathrm{H},-2.2803324958,0.79$ $51886467,1.5236674743 \backslash \mathrm{H},-1.3336626955,1.8291056989,-1.2259838927 \backslash \mathrm{H},-1$. $4051092731,2.7545746938,0.3784925218 \backslash \mathrm{H}, 0.3303115605,0.5215672598,0.081$ $1493877 \backslash \mathrm{Br}, 1.351776717,-0.4677253004,-0.028952227 \backslash \mathrm{H},-2.3019013225,-1.2$ $69881296,0.4480439907 \backslash \mathrm{H},-2.0240432223,-0.6003088188,-1.0630196371 \backslash \backslash$ Ver sion $=x 86-L$ inux-G03RevB.03 $\backslash$ State $=1-A \backslash H F=-2703.5697696 \backslash M P 2=-2704.1507463$ $\backslash \mathrm{RMSD}=8.742 \mathrm{e}-09 \backslash \mathrm{RMSF}=2.022 \mathrm{e}-05 \backslash \mathrm{Dipole}=-0.4260028,-0.1265545,0.1114269 \backslash$ $\mathrm{PG}=\mathrm{C} 01 \quad \mathrm{X}(\mathrm{C} 2 \mathrm{H} 6 \mathrm{Br} 1 \mathrm{~N} 1)] \backslash \backslash @$ 


\section{$\mathrm{NH}_{2} \mathrm{CHCH}_{2} \cdots \cdot \mathrm{HCl}(\mathrm{N} 1)$}

$1 \backslash 1 \backslash G I N C-L C 126 \backslash F O p t \backslash R M P 2-F C \backslash 6-31+G(d, p) \backslash C 2 H 6 C 11 N 1 \backslash B U C 562 \backslash 03-N o v-2004 \backslash 0$ $\backslash \backslash \#$ MP2 $/ 6-31+G(D, P)$ OPT MAXDISK $=1342177280 \backslash \backslash \mathrm{CH} 2 \mathrm{CHNH} 2-\mathrm{HF} \backslash \backslash 0,1 \backslash \mathrm{C}, 1.593$ $8362428,0.3531257647,0.4444075767 \backslash \mathrm{C}, 2.4661366697,-0.4348121202,-0.1994$ $875521 \backslash \mathrm{N}, 0.5724452953,1.1080870264,-0.1821781127 \backslash \mathrm{H}, 1.5633402948,0.3906$ $472577,1.526145785 \backslash \mathrm{H}, 2.4942416942,-0.4943364654,-1.2794717598 \backslash \mathrm{H}, 3.1561$ $947033,-1.0459487527,0.3602694011 \backslash \mathrm{H}, 0.4166063748,2.0043110554,0.265227$ $5985 \backslash \mathrm{H}, 0.7523070973,1.2550271996,-1.1701497217 \backslash \mathrm{H},-1.0053970717,0.13999$ $32385,-0.0726048494 \backslash \mathrm{Cl},-2.102602802,-0.5597755638,0.0103711875 \backslash \backslash$ Versio $\mathrm{n}=\mathrm{x} 86-\mathrm{Linux}-\mathrm{G} 03 \mathrm{RevB} .03 \backslash \mathrm{State}=1-\mathrm{A} \backslash \mathrm{HF}=-593.1560641 \backslash \mathrm{MP} 2=-593.7552109 \backslash \mathrm{RMSD}$ $=5.199 \mathrm{e}-09 \backslash \mathrm{RMSF}=2.041 \mathrm{e}-05 \backslash \mathrm{Dipole}=1.1614007,0.9996015,-0.1038164 \backslash \mathrm{PG}=\mathrm{C} 01$ $[\mathrm{X}(\mathrm{C} 2 \mathrm{H} 6 \mathrm{C} 11 \mathrm{~N} 1)] \backslash \backslash @$

\section{$\mathrm{NH}_{2} \mathrm{CHCH}_{2} \bullet \bullet \mathrm{HCl}(\mathrm{C} 3)$}

$1 \backslash 1 \backslash$ GINC-LC6 \FOpt $\backslash$ RMP $2-F C \backslash 6-31+G(d, p) \backslash C 2 H 6 C 11 N 1 \backslash B U C 562 \backslash 03-N o v-2004 \backslash 0 \backslash \backslash$

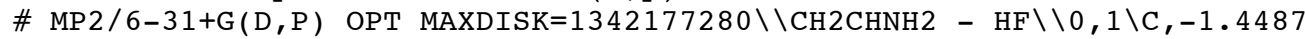
$867866,0.4853590539,0.4404749502 \backslash C,-0.9202914692,1.5559979223,-0.18900$ $3498 \backslash \mathrm{N},-1.8239884909,-0.7075017797,-0.159554854 \backslash \mathrm{H},-1.6537799135,0.5252$ $360094,1.5040586794 \backslash \mathrm{H},-0.736570906,1.5569501392,-1.255958977 \backslash \mathrm{H},-0.7602$ $896471,2.4736787475,0.3549087015 \backslash \mathrm{H}, 0.9332320937,0.2619381451,0.0719267$ $1 \backslash \mathrm{Cl}, 1.9071089403,-0.5719704848,-0.0227183337 \backslash \mathrm{H},-1.7438656841,-1.53192$ $53741,0.4156714533 \backslash \mathrm{H},-1.4771889575,-0.8580088243,-1.0963396292 \backslash \backslash$ Versio $\mathrm{n}=\mathrm{x} 86-\mathrm{Linux}-\mathrm{G} 03$ RevB. $03 \backslash$ State $=1-\mathrm{A} \backslash \mathrm{HF}=-593.1521234 \backslash \mathrm{MP} 2=-593.750907 \backslash \mathrm{RMSD}=$ $6.459 \mathrm{e}-09 \backslash \mathrm{RMSF}=3.848 \mathrm{e}-05 \backslash \mathrm{Dipole}=-0.5208961,-0.113001,0.1001746 \backslash \mathrm{PG}=\mathrm{C} 01$ $[\mathrm{X}(\mathrm{C} 2 \mathrm{H} 6 \mathrm{C} 11 \mathrm{~N} 1)] \backslash \backslash @$

\section{$\mathrm{NH}_{2} \mathrm{CHCH}_{2} \bullet \bullet \mathrm{H}-\mathrm{NC}(\mathrm{N} 1)$}

$1 \backslash 1 \backslash G I N C-S C 52 \backslash F O p t \backslash R M P 2-F C \backslash 6-31+G(d, p) \backslash C 3 H 6 N 2 \backslash B U C 562 \backslash 11-N o v-2004 \backslash 0 \backslash \backslash \#$ MP $2 / 6-31+\mathrm{G}(\mathrm{D}, \mathrm{P})$ OPT MAXDISK $=1342177280 \backslash \backslash \mathrm{CH} 2 \mathrm{CHNH} 2-\mathrm{H}-\mathrm{NC} \backslash \backslash 0,1 \backslash \mathrm{C}, 1.45366$ $61778,0.3072976993,0.4506165032 \backslash C, 2.3022038244,-0.5044923758,-0.194372$ $7527 \backslash \mathrm{N}, 0.450694561,1.0913809285,-0.1780951166 \backslash \mathrm{H}, 1.4271177712,0.3487281$ $328,1.5322885647 \backslash \mathrm{H}, 2.3271674293,-0.5652359517,-1.274399266 \backslash \mathrm{H}, 2.9771096$ $893,-1.133073846,0.3645191412 \backslash \mathrm{H}, 0.3312871314,1.9942222247,0.2683816156$ $\backslash \mathrm{H}, 0.6482307295,1.2393116767,-1.1630699365 \backslash \mathrm{H},-1.1291407434,0.152349887$ $9,-0.0799156066 \backslash N,-2.010767588,-0.3764468132,-0.0238137714 \backslash \mathrm{C},-3.032746$ $8054,-0.9762788121,0.0380158667 \backslash \backslash$ Version=DEC-AXP-OSF / 1-G03RevB.03 \Stat $\mathrm{e}=1-\mathrm{A} \backslash \mathrm{HF}=-225.9543271 \backslash \mathrm{MP} 2=-226.694142 \backslash \mathrm{RMSD}=2.951 \mathrm{e}-09 \backslash \mathrm{RMSF}=8.360 \mathrm{e}-05 \backslash \mathrm{Di}$ pole $=1.8507177,1.3187687,-0.131839 \backslash \mathrm{PG}=\mathrm{C} 01[\mathrm{X}(\mathrm{C} 3 \mathrm{H} 6 \mathrm{~N} 2)] \backslash \backslash @$

\section{$\mathrm{NH}_{2} \mathrm{CHCH}_{2} \cdots \mathbf{H}-\mathrm{NC}(\mathrm{C} 3)$}

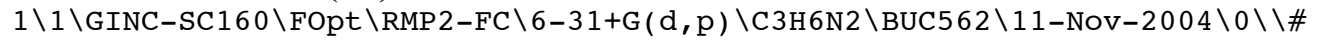
MP2 $/ 6-31+G(D, P)$ OPT MAXDISK $=1342177280 \backslash \backslash \mathrm{CH} 2 \mathrm{CHNH} 2-\mathrm{H}-\mathrm{NC} \backslash \backslash 0,1 \backslash \mathrm{C},-1.323$ $9861091,0.4650571765,0.4447628967 \backslash \mathrm{C},-0.7649313292,1.5312135234,-0.1695$ $02456 \backslash \mathrm{N},-1.6517153608,-0.7390952517,-0.1509940144 \backslash \mathrm{H},-1.591249213,0.522$ $3003148,1.4936503375 \backslash \mathrm{H},-0.52983188,1.5219821574,-1.2267302503 \backslash \mathrm{H},-0.653$ $7977927,2.4601952399,0.3673634016 \backslash \mathrm{H}, 1.0308723151,0.3088833252,0.092450$ $8538 \backslash \mathrm{N}, 1.8054827328,-0.3408726402,-0.0022312779 \backslash \mathrm{H},-1.6577535359,-1.552$ $8574895,0.4431910519 \backslash \mathrm{H},-1.267786126,-0.9167126483,-1.0676706715 \backslash \mathrm{C}, 2.68$ $77798763,-1.126939976,-0.1135400535 \backslash \backslash$ Version=DEC-AXP-OSF / 1-G03RevB.03\ State $=1-\mathrm{A} \backslash \mathrm{HF}=-225.948702 \backslash \mathrm{MP} 2=-226.6884286 \backslash \mathrm{RMSD}=7.703 \mathrm{e}-09 \backslash \mathrm{RMSF}=3.375 \mathrm{e}-0$ $5 \backslash$ Dipole $=-1.2136459,0.3230422,0.2052042 \backslash \mathrm{PG}=\mathrm{C} 01 \quad[\mathrm{X}(\mathrm{C} 3 \mathrm{H} 6 \mathrm{~N} 2)] \backslash \backslash @$

\section{$\mathrm{NH}_{2} \mathrm{CHCH}_{2} \cdots \cdot \mathrm{H}-\mathrm{CN}$ (N1)}

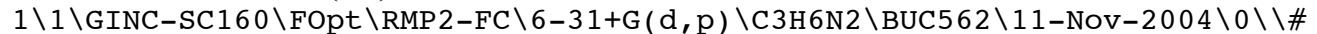
MP2 /6-31+G(D,P) OPT MAXDISK $=1342177280 \backslash \backslash \mathrm{CH} 2 \mathrm{CHNH} 2-\mathrm{H}-\mathrm{CN} \backslash \backslash 0,1 \backslash \mathrm{C}, 1.5165$ $826617,0.4124476254,0.4448257251 \backslash \mathrm{C}, 2.250961294,-0.5128020955,-0.191794$ $0479 \backslash \mathrm{N}, 0.6168948562,1.3042823052,-0.1778279447 \backslash \mathrm{H}, 1.5119169744,0.469498$ $1186,1.5266383038 \backslash \mathrm{H}, 2.2590866385,-0.5943959271,-1.2707718778 \backslash \mathrm{H}, 2.84671$ $20237,-1.2111114423,0.3741909055 \backslash \mathrm{H}, 0.5587859741,2.2013314812,0.2875025$ $878 \backslash \mathrm{H}, 0.8207344712,1.4462932571,-1.1607421067 \backslash \mathrm{H},-1.181090856,0.1205497$ $88,-0.0716295971 \backslash \mathrm{C},-2.0679144128,-0.49086205,-0.0265424684 \backslash \mathrm{N},-3.047455$ $2109,-1.1449774702,0.0286674492 \backslash \backslash$ Version=DEC-AXP-OSF / 1-G03RevB.03 \Stat $\mathrm{e}=1-\mathrm{A} \backslash \mathrm{HF}=-225.9641231 \backslash \mathrm{MP} 2=-226.7165724 \backslash \mathrm{RMSD}=4.810 \mathrm{e}-09 \backslash \mathrm{RMSF}=1.600 \mathrm{e}-04 \backslash \mathrm{D}$ ipole $=1.5118347,1.296459,-0.0988453 \backslash P G=C 01 \quad[X(C 3 H 6 N 2)] \backslash \backslash @$ 


\section{$\mathrm{NH}_{2} \mathrm{CHCH}_{2} \cdots \mathbf{H}-\mathrm{CN}(\mathrm{N} 1)$}

$1 \backslash 1 \backslash$ GINC-SC160 $\mathrm{FOpt} \backslash \mathrm{RMP} 2-\mathrm{FC} \backslash 6-31+\mathrm{G}(\mathrm{d}, \mathrm{p}) \backslash \mathrm{C} 3 \mathrm{H} 6 \mathrm{~N} 2 \backslash \mathrm{BUC5} 62 \backslash 11-\mathrm{Nov}-2004 \backslash 0 \backslash \backslash \#$ MP2 $/ 6-31+G(D, P)$ OPT MAXDISK=1342177280 \\CH2CHNH2 - H-CN $\backslash \backslash 0,1 \backslash C,-1.395$ $6205344,0.5672673566,0.4509466768 \backslash C,-0.9318898297,1.6705552968,-0.1709$ $174552 \backslash \mathrm{N},-1.5984187316,-0.6749240273,-0.1336724626 \backslash \mathrm{H},-1.6972728445,0.6$ $145586793,1.4908939943 \backslash \mathrm{H},-0.6509104577,1.6621426743,-1.2166147265 \backslash \mathrm{H},-0$ $.9090548973,2.6130816164,0.352975651 \backslash \mathrm{H}, 1.142884764,0.3441915736,0.1057$ $85409 \backslash \mathrm{C}, 1.8797309784,-0.4288859572,-0.0030256707 \backslash \mathrm{H},-1.5182151344,-1.47$ $74495364,0.4707900498 \backslash \mathrm{H},-1.1862784258,-0.8219980057,-1.0435209725 \backslash \mathrm{N}, 2$. $6706363473,-1.294811284,-0.126660496 \backslash \backslash$ Version=DEC-AXP-OSF / 1-G03RevB.03 $\backslash$ State $=1-\mathrm{A} \backslash \mathrm{HF}=-225.9600795 \backslash \mathrm{MP} 2=-226.7135024 \backslash \mathrm{RMSD}=4.430 \mathrm{e}-09 \backslash \mathrm{RMSF}=1.313 \mathrm{e}$ $-05 \backslash \mathrm{Dipole}=-0.837314,0.3764183,0.2152393 \backslash \mathrm{PG}=\mathrm{C} 01 \quad[\mathrm{X}(\mathrm{C} 3 \mathrm{H} 6 \mathrm{~N} 2)] \backslash \backslash @$

\section{$\mathrm{C}_{4} \mathrm{H}_{4} \mathrm{O} \cdots \cdot \mathrm{H}_{2} \mathrm{~F}^{+}(\mathrm{O1})$}

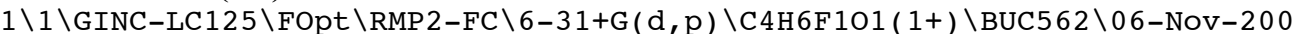
$4 \backslash 0 \backslash \backslash \#$ MP2 $/ 6-31+G(D, P) \quad O P T$ SYMMETRY=LOOSE MAXDISK $=1342177280 \backslash \backslash \mathrm{C} 4 \mathrm{H} 40-\mathrm{H}$ $2 \mathrm{~F}+(0) \backslash \backslash 1,1 \backslash 0,0,0.4077795835,-0.2861689577 \backslash \mathrm{C}, 1.1618899729,0.123810436$ $4,0.5029327045 \backslash \mathrm{C}, 0.7233906528,-0.2191025508,1.7290764244 \backslash \mathrm{C},-1.16188997$ $29,0.1238104364,0.5029327045 \backslash C,-0.7233906528,-0.2191025508,1.729076424$ $4 \backslash \mathrm{H}, 2.1016626043,0.2817355977,0.0038994156 \backslash \mathrm{H}, 1.3631399269,-0.446445182$ $4,2.5659673589 \backslash \mathrm{H},-1.3631399269,-0.4464451824,2.5659673589 \backslash \mathrm{H},-2.1016626$ $043,0.2817355977,0.0038994156 \backslash \mathrm{H}, 0 ., 0.2013296036,-1.2713270505 \backslash \mathrm{F}, 0 .,-0$. $2442168805,-2.7542234093 \backslash \mathrm{H}, 0 ., 0.2073101951,-3.5751537006 \backslash \backslash$ Version $=\mathrm{x} 86-$ Linux-G03RevB.03 State $=1-A^{\prime} \backslash \mathrm{HF}=-328.9657142 \backslash \mathrm{MP} 2=-329.8686225 \backslash \mathrm{RMSD}=6.87$ $5 e-09 \backslash \mathrm{RMSF}=3.917 \mathrm{e}-05 \backslash \mathrm{Dipole}=0,0.4119592,-0.9077232 \backslash \mathrm{PG}=\mathrm{CS} \quad[\mathrm{SG}(\mathrm{H} 2 \mathrm{~F} 101)$, $\mathrm{X}(\mathrm{C} 4 \mathrm{H} 4) \mathrm{J} \backslash \backslash \mathrm{Q}$

\section{$\mathrm{C}_{4} \mathrm{H}_{4} \mathrm{O} \cdots \cdot \mathrm{H}_{2} \mathrm{~F}^{+}(\mathrm{C} 2)$}

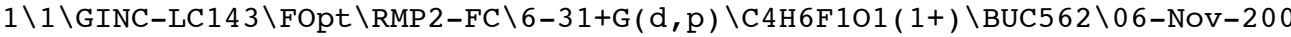
$4 \backslash 0 \backslash \backslash \#$ MP2 $/ 6-31+G(D, P) \quad O P T$ MAXDISK $=1342177280 \backslash \backslash \mathrm{C} 4 \mathrm{H} 4 \mathrm{O}-\mathrm{H} 2 \mathrm{~F}+\backslash \backslash 1,1 \backslash \mathrm{O},-0.8$ $504180206,0.6677315932,-0.6652146277 \backslash \mathrm{C},-0.4233471828,-0.6813950591,-1$. $0451522421 \backslash \mathrm{C}, 1.006125958,-0.7147794083,-0.6784638115 \backslash \mathrm{C}, 1.3516087005,0$. $4840447761,-0.1301396356 \backslash \mathrm{C}, 0.1665055887,1.2701738455,-0.150394008 \backslash \mathrm{H},-1$ $.0473818308,-1.3650225323,-0.4666035965 \backslash \mathrm{H}, 2.3076785149,0.8021035901,0$. $2525182952 \backslash \mathrm{H}, 0.0015405304,2.2830949001,0.1946747798 \backslash \mathrm{H}, 1.643196933,-1.5$ $749107088,-0.828743627 \backslash \mathrm{H},-0.6317783564,-0.7828198706,-2.1118569082 \backslash \mathrm{F},-$ $0.7712971167,-0.6635070523,1.9476488112 \backslash \mathrm{H},-1.1335959625,-0.8809995791$, $2.7777869607 \backslash \backslash$ Version $=x 86-L$ inux-G03RevB.03 $\backslash$ State $=1-\mathrm{A} \backslash \mathrm{HF}=-329.0120061 \backslash \mathrm{M}$ $\mathrm{P} 2=-329.9036797 \backslash \mathrm{RMSD}=3.898 \mathrm{e}-09 \backslash \mathrm{RMSF}=1.789 \mathrm{e}-05 \backslash \mathrm{Dipole}=0.4794202,-0.0722$ $912,-0.071388 \backslash \mathrm{PG}=\mathrm{C} 01 \quad[\mathrm{X}(\mathrm{C} 4 \mathrm{H} 6 \mathrm{~F} 101)] \backslash \backslash @$

\section{$\mathrm{C}_{4} \mathrm{H}_{4} \mathrm{O} \cdots \cdot \mathrm{H}_{2} \mathrm{~F}^{+}(\mathrm{C} 3)$}

$1 \backslash 1 \backslash$ GINC-LC79 $\backslash$ FOpt $\backslash R M P 2-F C \backslash 6-31+G(d, p) \backslash C 4$ H6F $101(1+) \backslash B U C 562 \backslash 06-N o v-2004$ $\backslash 0 \backslash \backslash \#$ MP2 $/ 6-31+G(D, P) \quad O P T$ MAXDISK $=1342177280 \backslash \backslash \mathrm{C} 4 \mathrm{H} 4 \mathrm{O}-\mathrm{H} 2 \mathrm{~F}+(\mathrm{O}) \backslash \backslash 1,1 \backslash \mathrm{O}, 1$. $0440285073,-0.7197570842,-0.7797951679 \backslash \mathrm{C}, 0.080171606,-0.7118169946,0.0$ $586589488 \backslash \mathrm{C},-0.0058042099,0.5591964153,0.7803540782 \backslash \mathrm{C}, 1.1349729327,1.3$ $242119048,0.1970747475 \backslash \mathrm{C}, 1.7309266968,0.5512558703,-0.7186747292 \backslash \mathrm{H}, 1.4$ $268362197,2.3271197903,0.4630516365 \backslash \mathrm{H}, 2.5597468379,0.6235020864,-1.400$ $4452847 \backslash \mathrm{H}, 0.0544643259,0.3939201417,1.8642927316 \backslash \mathrm{H},-0.5362166065,-1.59$ $81558255,0.1236713696 \backslash \mathrm{H},-0.9945065386,1.0060795244,0.6075214268 \backslash \mathrm{F},-2.7$ $593406862,-0.7137951923,0.2712402149 \backslash \mathrm{H},-3.6700882749,-0.9073354873,0.2$ $346292576 \backslash \backslash$ Version $=x 86-L$ inux-G03RevB.03 $\backslash$ State $=1-A \backslash H F=-328.9910362 \backslash \mathrm{MP} 2=$ $-329.8844322 \backslash \mathrm{RMSD}=6.302 \mathrm{e}-09 \backslash \mathrm{RMSF}=1.883 \mathrm{e}-05 \backslash \mathrm{Dipole}=-0.155832,0.1395,0.3$ $427781 \backslash \mathrm{PG}=\mathrm{C} 01 \quad[\mathrm{X}(\mathrm{C} 4 \mathrm{H} 6 \mathrm{~F} 101)] \backslash \backslash @$

\section{$\mathrm{C}_{4} \mathrm{H}_{4} \mathrm{O} \cdots \bullet \mathrm{H}_{2} \mathrm{Cl}^{+}(\mathrm{O} 1)$}

$1 \backslash 1 \backslash$ GINC-LC1 $15 \backslash F O p t \backslash R M P 2-F C \backslash 6-31+G(d, p) \backslash C 4$ H6Cl101 (1+) \BUC562\06-Nov-20 $04 \backslash 0 \backslash \backslash \#$ MP2 $/ 6-31+G(D, P)$ OPT SYMMETRY=LOOSE MAXDISK=1342177280 \C4H4O $\mathrm{H} 2 \mathrm{Cl}+\backslash \backslash 1,1 \backslash \mathrm{O}, 0 ., 0.4215597143,-0.254098785 \backslash \mathrm{C},-1.1610994733,0.121061897$, $-1.0360167248 \backslash \mathrm{C}, 1.1610994733,0.121061897,-1.0360167248 \backslash \mathrm{C},-0.7233652875$ $,-0.2487798032,-2.2551519866 \backslash \mathrm{C}, 0.7233652875,-0.2487798032,-2.255151986$ $6 \backslash \mathrm{H},-2.101336906,0.2919992131,-0.5418968021 \backslash \mathrm{H}, 2.101336906,0.2919992131$ $,-0.5418968021 \backslash \mathrm{H},-1.3630790204,-0.4950866785,-3.0866661701 \backslash \mathrm{H}, 1.3630790$ $204,-0.4950866785,-3.0866661701 \backslash \mathrm{H}, 0$. $, 0.2394841403,0.7477380469 \backslash \mathrm{Cl}, 0$. ,$0.1570115042,2.6380900888 \backslash \mathrm{H}, 0 ., 0.9960235231,3.1886712044 \backslash \backslash$ Version $=\mathrm{x} 86-$ Linux-G03RevB. 03 $\backslash$ State $=1-A^{\prime} \backslash \mathrm{HF}=-688.9987208 \backslash \mathrm{MP} 2=-689.8559186 \backslash \mathrm{RMSD}=6.86$ $4 \mathrm{e}-09 \backslash \mathrm{RMSF}=2.783 \mathrm{e}-05 \backslash \mathrm{Dipole}=0,0.5678827,-0.2296927 \backslash \mathrm{PG}=\mathrm{CS} \quad[\mathrm{SG}(\mathrm{H} 2 \mathrm{Cl} 1 \mathrm{O} 1)$ , $\mathrm{X}(\mathrm{C} 4 \mathrm{H} 4) \mathrm{]} \backslash \backslash @$ 


\section{$\mathrm{C}_{4} \mathrm{H}_{4} \mathrm{O} \cdots \mathrm{H}_{2} \mathrm{Cl}^{+}(\mathrm{C} 2)$}

$1 \backslash 1 \backslash$ GINC-LC8 $1 \backslash F$ Opt $\backslash$ RMP2-FC $\backslash 6-31+G(d, p) \backslash C 4 H 6 C 1101(1+) \backslash B U C 562 \backslash 06-N o v-200$ $4 \backslash 0 \backslash \backslash \#$ MP2 $/ 6-31+G(D, P) \quad O P T$ MAXDISK $=1342177280 \backslash \backslash \mathrm{C} 4 \mathrm{H} 4 \mathrm{O}-\mathrm{H} 2 \mathrm{Cl}+\backslash \backslash 1,1 \backslash \mathrm{O},-0$. $8401774127,0.7152598602,-1.0564127084 \backslash \mathrm{C},-0.1668118169,-0.4547573372,-1$ $.6253187944 \backslash C, 1.2393583287,-0.2913767976,-1.2055791685 \backslash C, 1.3505587175$, $0.8461391406,-0.4626086909 \backslash \mathrm{C}, 0.0408895055,1.4008317609,-0.4113199766 \backslash \mathrm{H}$ $,-0.6679259611,-1.3270661727,-1.1993591498 \backslash \mathrm{H}, 2.2266288685,1.2655976934$ $, 0.0051723842 \backslash \mathrm{H},-0.3120381624,2.2979068865,0.0815914776 \backslash \mathrm{H}, 2.0258199974$ $,-0.9878565778,-1.4605746152 \backslash \mathrm{H},-0.3346050748,-0.4146393306,-2.70351257$ $42 \backslash \mathrm{Cl},-0.5504618956,-0.8544082158,1.9626122484 \backslash \mathrm{H},-1.642576549,-1.03610$ $23113,2.5925357031 \backslash \backslash$ Version $=x 86-$ Linux-G03RevB.03 $\backslash$ State $=1-A \backslash H F=-689.049$ $1679 \backslash \mathrm{MP} 2=-689.8911269 \backslash \mathrm{RMSD}=9.663 \mathrm{e}-09 \backslash \mathrm{RMSF}=9.639 \mathrm{e}-06 \backslash \mathrm{Dipole}=0.4471727,0$ $.5165571,-1.2700918 \backslash \mathrm{PG}=\mathrm{C} 01[\mathrm{X}(\mathrm{C} 4 \mathrm{H} 6 \mathrm{Cl} 1 \mathrm{O} 1)] \backslash \backslash @$

\section{$\mathrm{C}_{4} \mathrm{H}_{4} \mathrm{O} \cdots \mathrm{H}_{2} \mathrm{Cl}^{+}(\mathrm{C} 3)$}

$1 \backslash 1 \backslash$ GINC-LC66 \FOpt \RMP2-FC $\backslash 6-31+G(d, p) \backslash C 4 H 6 C 1101(1+) \backslash B U C 562 \backslash 07-N o v-200$ $4 \backslash 0 \backslash \backslash \#$ MP2 /6-31+G(D,P) OPT MAXDISK=1342177280 \C4H4O $-\mathrm{H} 2 \mathrm{Cl}+\backslash \backslash 1,1 \backslash 0,1.0$ $848880726,-0.8002432328,-0.684349415 \backslash \mathrm{C}, 0.729670811,-1.1330695459,0.494$ $9515089 \backslash \mathrm{C}, 0.8164669961,-0.0097849124,1.4322471501 \backslash \mathrm{C}, 1.2647754478,1.098$ $7884147,0.5391555428 \backslash \mathrm{C}, 1.4200586785,0.6038392203,-0.6953069438 \backslash \mathrm{H}, 1.443$ $4123403,2.118793997,0.8387503406 \backslash \mathrm{H}, 1.7170881814,0.9665927952,-1.663518$ $2721 \backslash \mathrm{H}, 1.538093003,-0.254543113,2.225836384 \backslash \mathrm{H}, 0.44114939,-2.1643501819$ $, 0.6535221627 \backslash \mathrm{H},-0.1413432062,0.1583309113,1.9388060808 \backslash \mathrm{Cl},-2.11421778$ $31,0.1657342417,-0.4995669918 \backslash \mathrm{H},-3.1216335766,-0.5989997158,-0.6522460$ $635 \backslash \backslash$ Version $=x 86-$ Linux-G03RevB.03 $\backslash$ State $=1-A \backslash H F=-689.0260105 \backslash M P 2=-689.8$ $718744 \backslash \mathrm{RMSD}=5.114 \mathrm{e}-09 \backslash \mathrm{RMSF}=2.503 \mathrm{e}-05 \backslash \mathrm{Dipole}=1.0836351,-0.6588278,1.011$ $6683 \backslash \mathrm{PG}=\mathrm{C} 01 \quad[\mathrm{X}(\mathrm{C} 4 \mathrm{H} 6 \mathrm{C} 1101)] \backslash \backslash @$

\section{$\mathrm{C}_{4} \mathrm{H}_{4} \mathrm{O} \cdots \cdot \mathrm{H}_{2} \mathrm{Br}^{+}(\mathrm{O1})$}

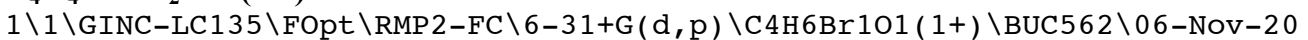
$04 \backslash 0 \backslash \backslash \#$ MP2/6-31+G(D,P) OPT SYMMETRY=LOOSE MAXDISK=1342177280 \C4H4O $\mathrm{H} 2 \mathrm{Br}+\backslash \backslash 1,1 \backslash \mathrm{O}, 0 ., 0.4180109419,-0.9675739107 \backslash \mathrm{C},-1.1595512656,0.116287204$ $7,-1.7472327666 \backslash \mathrm{C}, 1.1595512656,0.1162872047,-1.7472327666 \backslash \mathrm{C},-0.7229955$ $193,-0.2570935355,-2.9664636422 \backslash \mathrm{C}, 0.7229955193,-0.2570935355,-2.966463$ $6422 \backslash \mathrm{H},-2.1001935788,0.2878935649,-1.2540539986 \backslash \mathrm{H}, 2.1001935788,0.28789$ $35649,-1.2540539986 \backslash \mathrm{H},-1.363765932,-0.5058286588,-3.7963669867 \backslash \mathrm{H}, 1.363$ $765932,-0.5058286588,-3.7963669867 \backslash \mathrm{H}, 0 ., 0.259227108,0.0478504006 \backslash \mathrm{Br}, 0$. $,-0.0786157899,2.0538148142 \backslash \mathrm{H}, 0 ., 1.2737841622,2.4744212649 \backslash \backslash$ Version $=\mathrm{x} 8$ 6-Linux-G03RevB.03 \State $=1-A^{\prime} \backslash \mathrm{HF}=-2799.4194538 \backslash \mathrm{MP} 2=-2800.2575609 \backslash \mathrm{RMSD}=$ $8.690 \mathrm{e}-09 \backslash \mathrm{RMSF}=3.345 \mathrm{e}-05 \backslash \mathrm{Dipole}=0 ., 0.4941935,-1.4246535 \backslash \mathrm{PG}=\mathrm{CS} \quad[\mathrm{SG}(\mathrm{H} 2 \mathrm{Br}$ $101), \mathrm{X}(\mathrm{C} 4 \mathrm{H} 4)] \backslash \backslash @$

\section{$\mathrm{C}_{4} \mathrm{H}_{4} \mathrm{O} \cdots \cdot \mathrm{H}_{2} \mathrm{Br}^{+}(\mathrm{C} 2)$}

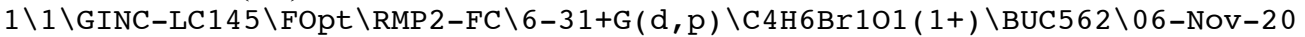
$04 \backslash 0 \backslash \backslash \#$ MP $2 / 6-31+\mathrm{G}(\mathrm{D}, \mathrm{P})$ OPT MAXDISK $=1342177280 \backslash \backslash \mathrm{C} 4 \mathrm{H} 4 \mathrm{O}-\mathrm{H} 2 \mathrm{Br}+\backslash \backslash 1,1 \backslash \mathrm{O},-0$ $.750225031,0.4807607844,-1.6896334903 \backslash \mathrm{C}, 0.0751270019,-0.5022507259,-2$. $3956495718 \backslash \mathrm{C}, 1.4572465455,-0.1362862574,-2.0209422971 \backslash \mathrm{C}, 1.4184242937,0$ $.9255549512,-1.1685333585 \backslash \mathrm{C}, 0.0375543027,1.2338122695,-1.0004420423 \backslash \mathrm{H}$, $-0.2470780493,-1.4815948756,-2.0328573887 \backslash \mathrm{H}, 2.2374361667,1.4455005462$, $-0.6981461217 \backslash \mathrm{H},-0.4269621621,2.0158399039,-0.4151216148 \backslash \mathrm{H}, 2.333702312$ $1,-0.6550399022,-2.3832613948 \backslash \mathrm{H},-0.1595228626,-0.4011233033,-3.4568261$ $86 \backslash \mathrm{Br},-0.3975885094,-0.3885771263,1.7116804753 \backslash \mathrm{H},-1.750290192,-0.29445$ $06483,2.1078676117 \backslash \backslash$ Version $=x 86-$ Linux-G03RevB.03 $\backslash$ State $=1-A \backslash H F=-2799.46$ $95375 \backslash \mathrm{MP} 2=-2800.2922526 \backslash \mathrm{RMSD}=9.564 \mathrm{e}-09 \backslash \mathrm{RMSF}=2.197 \mathrm{e}-06 \backslash \mathrm{Dipole}=0.7144378$ $, 0.6020347,-2.5981032 \backslash \mathrm{PG}=\mathrm{C} 01[\mathrm{X}(\mathrm{C} 4 \mathrm{H} 6 \mathrm{Br} 101)] \backslash \backslash @$

\section{$\mathrm{C}_{4} \mathrm{H}_{4} \mathrm{O} \cdot \bullet \cdot \mathrm{H}_{2} \mathrm{Br}^{+}(\mathrm{C3})$}

$1 \backslash 1 \backslash$ GINC-LC122\FOpt \RMP2-FC \6-31+G(d,p) \C4H6Br1O1( $1+) \backslash B U C 562 \backslash 07-N o v-20$ $04 \backslash 0 \backslash \backslash \#$ MP2 /6-31+G(D,P) OPT MAXDISK $=1342177280 \backslash \backslash \mathrm{C} 4 \mathrm{H} 4 \mathrm{O}-\mathrm{H} 2 \mathrm{Br}+\backslash \backslash 1,1 \backslash \mathrm{O}, 2$. $6884609324,-0.9595863011,-0.432558118 \backslash \mathrm{C}, 1.8525446875,-1.2325750456,0.4$ $960484953 \backslash \mathrm{C}, 1.2849497619,-0.0264688319,1.0910942427 \backslash \mathrm{C}, 1.9953922674,1.0$ $513976642,0.3467790003 \backslash \mathrm{C}, 2.803300938,0.4742243876,-0.5525387635 \backslash \mathrm{H}, 1.86$ $47745116,2.111658306,0.4910843983 \backslash \mathrm{H}, 3.4954903404,0.7870832683,-1.31425$ $79833 \backslash \mathrm{H}, 1.4012066622,-0.0133837569,2.1816460086 \backslash \mathrm{H}, 1.6636141156,-2.2795$ $750362,0.697530508 \backslash \mathrm{H}, 0.1944454356,-0.0263820098,0.9090766935 \backslash \mathrm{Br},-2.133$ $1188208,0.1598431578,-0.2445419632 \backslash \mathrm{H},-3.0851857257,-0.096689931,0.7660$ $561841 \backslash \backslash$ Version $=x 86-$ Linux-G03RevB.03 $\backslash$ State $=1-A \backslash H F=-2799.4437637 \backslash M P 2=-2$ $800.2700462 \backslash \mathrm{RMSD}=3.506 \mathrm{e}-09 \backslash \mathrm{RMSF}=2.048 \mathrm{e}-05 \backslash \mathrm{Dipole}=2.6987889,-0.4921342$, $1.1885038 \backslash \mathrm{PG}=\mathrm{C} 01 \quad[\mathrm{X}(\mathrm{C} 4 \mathrm{H} 6 \mathrm{Br} 1 \mathrm{O} 1)] \backslash \backslash @$ 


\section{$\mathrm{C}_{4} \mathrm{H}_{4} \mathrm{O} \cdots \mathrm{H}_{3} \mathrm{O}^{+}(\mathrm{O} 1)$}

$1 \backslash 1 \backslash$ GINC-SC20 $\mathrm{FOpt} \backslash \mathrm{RMP} 2-\mathrm{FC} \backslash 6-31+\mathrm{G}(\mathrm{d}, \mathrm{p}) \backslash \mathrm{C} 4 \mathrm{H} 7 \mathrm{O} 2(1+) \backslash \mathrm{BUC} 562 \backslash 08-\mathrm{Nov}-2004 \backslash 0$ $\backslash \backslash \#$ MP2 $/ 6-31+G(D, P)$ OPT MAXDISK $=1342177280 \backslash \backslash C 4 \mathrm{H} 4 \mathrm{O}-\mathrm{H} 3 \mathrm{O}+\backslash \backslash 1,1 \backslash 0,0.02733$ $06998,-0.1205947515,0.343856593 \backslash C,-1.1377839721,-0.0779571461,-0.44776$ $42128 \backslash \mathrm{C},-0.7557116836,-0.0528934491,-1.7458294469 \backslash \mathrm{C}, 1.1421310893,-0.09$ $44492956,-0.5166987729 \backslash \mathrm{C}, 0.6826424509,-0.0636936249,-1.7894237537 \backslash \mathrm{H},-2$ $.068022618,-0.090563122,0.0921726458 \backslash \mathrm{H},-1.4271917914,-0.0334699258,-2$. $5878991784 \backslash \mathrm{H}, 1.3023228795,-0.0540297494,-2.6705030769 \backslash \mathrm{H}, 2.104718348,-0$ $.1256777906,-0.037883377 \backslash \mathrm{H}, 0.0431086086,0.0820559705,1.5017960504 \backslash 0,-0$ $.0277046873,0.276671733,2.6973597772 \backslash \mathrm{H}, 0.2334381754,1.1376806354,3.060$ $1550617 \backslash \mathrm{H}, 0.2269509904,-0.4306507752,3.3107280303 \backslash \backslash$ Version=DEC-AXP-OSF $/ 1-G 03$ RevB.03 \State $=1-A \backslash H F=-304.9905452 \backslash M P 2=-305.9128729 \backslash$ RMSD $=4.207 e-0$ $9 \backslash \mathrm{RMSF}=9.461 \mathrm{e}-06 \backslash \mathrm{Dipole}=0.3988134,0.2776913,2.2239109 \backslash \mathrm{PG}=\mathrm{C} 01 \quad[\mathrm{X}(\mathrm{C} 4 \mathrm{H} 7 \mathrm{O} 2$ )$] \backslash \backslash @$

\section{$\mathrm{C}_{4} \mathrm{H}_{4} \mathrm{O} \bullet \bullet \mathrm{H}_{3} \mathrm{O}^{+}(\mathrm{C} 2)$}

$1 \backslash 1 \backslash$ GINC-LC99 \FOpt \RMP2-FC $\backslash 6-31+\mathrm{G}(\mathrm{d}, \mathrm{p}) \backslash \mathrm{C} 4 \mathrm{H} 7 \mathrm{O} 2(1+) \backslash \mathrm{BUC} 562 \backslash 06-\mathrm{Nov}-2004 \backslash 0$ $\backslash \backslash \#$ MP2 $/ 6-31+G(D, P) \quad O P T$ MAXDISK $=1342177280 \backslash \backslash \mathrm{C} 4 \mathrm{H} 4 \mathrm{O}-\mathrm{H} 3 \mathrm{O}+\backslash \backslash 1,1 \backslash \mathrm{O},-1.0015$ $609092,0.404603873,-0.554966828 \backslash \mathrm{C},-0.4502842099,-0.7787686573,-1.21946$ $14889 \backslash \mathrm{C}, 1.0075274409,-0.652817365,-1.0089782966 \backslash \mathrm{C}, 1.2525561287,0.46213$ $7133,-0.2694343301 \backslash \mathrm{C},-0.0238785596,1.0420177572,-0.0113351271 \backslash \mathrm{H},-0.899$ $9808787,-1.6378785605,-0.7171621664 \backslash \mathrm{H}, 2.1968341043,0.8531113683,0.0724$ $990008 \backslash \mathrm{H},-0.2743174806,1.9386804309,0.5377243169 \backslash \mathrm{H}, 1.7303409197,-1.360$ $0846278,-1.3900099735 \backslash \mathrm{H},-0.7765672183,-0.7348618817,-2.2593866175 \backslash 0,-0$ $.4449960031,-0.2446840665,2.2124594421 \backslash \mathrm{H},-1.2992609007,-0.226791306,2$. $6659755346 \backslash \mathrm{H}, 0.1798819524,-0.5469470827,2.8856744479 \backslash \backslash$ Version=x86-Linu $\mathrm{X}-\mathrm{G} 03$ RevB. 03 $\backslash$ State $=1-\mathrm{A} \backslash \mathrm{HF}=-305.0255927 \backslash \mathrm{MP} 2=-305.9282016 \backslash \mathrm{RMSD}=9.707 \mathrm{e}-09$ $\backslash \mathrm{RMSF}=1.911 \mathrm{e}-05 \backslash \mathrm{Dipole}=0.4531254,-0.1903572,-0.0049054 \backslash \mathrm{PG}=\mathrm{C} 01 \quad[\mathrm{X}(\mathrm{C} 4 \mathrm{H} 70$ 2) $] \backslash \backslash @$

\section{$\mathrm{C}_{4} \mathrm{H}_{4} \mathrm{O} \cdots \cdot \mathrm{H}_{3} \mathrm{O}^{+}(\mathrm{C} 3)$}

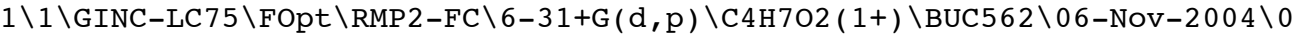
$\backslash \backslash \#$ MP $2 / 6-31+G(D, P)$ OPT MAXDISK $=1342177280 \backslash \backslash \mathrm{C} 4 \mathrm{H} 4 \mathrm{O}-\mathrm{H} 3 \mathrm{O}+\backslash \backslash 1,1 \backslash \mathrm{O}, 0.92537$ $1492,-0.8396116902,-0.7028726109 \backslash \mathrm{C}, 0.3585446238,-1.08041127,0.50413153$ $53 \backslash C, 0.1645819566,0.110933693,1.1822569722 \backslash C, 0.6978541594,1.1420506284$ $, 0.3326732304 \backslash \mathrm{C}, 1.1392933177,0.510469057,-0.798902339 \backslash \mathrm{H}, 0.7679012724,2$ $.196118683,0.5462665274 \backslash \mathrm{H}, 1.6121930871,0.8437615969,-1.7066202962 \backslash \mathrm{H},-0$ $.1589588146,0.2044230646,2.2085319781 \backslash \mathrm{H}, 0.1802951928,-2.1145702591,0.7$ $51050258 \backslash \mathrm{H},-1.3123532945,-0.0267726012,0.2239143527 \backslash \mathrm{O},-2.1672278804,0$. $1027809265,-0.387428579 \backslash \mathrm{H},-2.9615977849,-0.3478248885,-0.0439196387 \backslash \mathrm{H}$, $-2.3542728955,1.0412578634,-0.5777700553 \backslash \backslash$ Version=x86-Linux-G03RevB.03 $\backslash$ State $=1-\mathrm{A} \backslash \mathrm{HF}=-304.9738448 \backslash \mathrm{MP} 2=-305.9018252 \backslash \mathrm{RMSD}=5.104 \mathrm{e}-09 \backslash \mathrm{RMSF}=1.571 \mathrm{e}$ $-05 \backslash \mathrm{Dipole}=-2.830359,0.5967323,0.2556426 \backslash \mathrm{PG}=\mathrm{C} 01[\mathrm{X}(\mathrm{C} 4 \mathrm{H} 7 \mathrm{O} 2)] \backslash \backslash @$

\section{$\mathrm{C}_{4} \mathrm{H}_{4} \mathrm{O} \cdots \cdot \mathrm{H}-\mathrm{NCH}^{+}(\mathrm{O} 1)$}

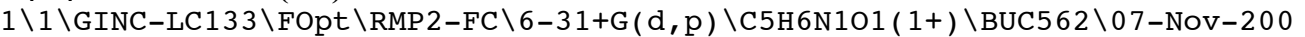
$4 \backslash 0 \backslash \backslash \#$ MP2 /6-31+G(D,P) OPT MAXDISK $=1342177280 \backslash \backslash \mathrm{C} 4 \mathrm{H} 4 \mathrm{O}-\mathrm{HNCH}+\backslash \backslash 1,1 \backslash \mathrm{O}, 0$. , $-0.3175850891,0.0557418154 \backslash C,-1.1433267467,-0.1541612219,0.8683481937 \backslash$ $\mathrm{C}, 1.1433267467,-0.1541612219,0.8683481937 \backslash \mathrm{C},-0.7204017586,0.049899871$, $2.1366938911 \backslash \mathrm{C}, 0.7204017586,0.049899871,2.1366938911 \backslash \mathrm{H},-2.0888954679,-$ $0.2530838584,0.3652603375 \backslash \mathrm{H}, 2.0888954679,-0.2530838584,0.3652603375 \backslash \mathrm{H}$, $-1.3653439669,0.1795207041,2.9897834041 \backslash \mathrm{H}, 1.3653439669,0.1795207041,2$. $9897834041 \backslash \mathrm{H}, 0 .,-0.0864413814,-1.05565564 \backslash \mathrm{N}, 0 ., 0.1643385788,-2.3635391$ $213 \backslash \mathrm{C}, 0.0 .3822559814,-3.508860757 \backslash \mathrm{H}, 0.0 .5814786726,-4.5629329937 \backslash \backslash \mathrm{Ve}$ rsion $=x 86-L$ inux-G03RevB.03 $\backslash$ State $=1-A^{\prime} \backslash H F=-321.8344274 \backslash M P 2=-322.8493628$ $\backslash \mathrm{RMSD}=9.693 \mathrm{e}-09 \backslash \mathrm{RMSF}=8.398 \mathrm{e}-06 \backslash \mathrm{Dipole}=0,0.3191468,-2.1589509 \backslash \mathrm{PG}=\mathrm{CS} \quad[\mathrm{S}$ $\mathrm{G}(\mathrm{C} 1 \mathrm{H} 2 \mathrm{~N} 1 \mathrm{O} 1), \mathrm{X}(\mathrm{C} 4 \mathrm{H} 4)] \backslash \backslash @$ 


\section{$\mathrm{C}_{4} \mathrm{H}_{4} \mathrm{O} \cdots \cdot \mathrm{H}-\mathrm{NCH}^{+}(\mathrm{C} 2)$}

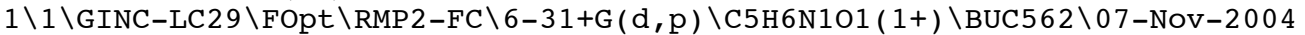
$\backslash 0 \backslash \backslash \#$ MP2 $/ 6-31+G(D, P)$ OPT MAXDISK $=1342177280 \backslash \backslash \mathrm{C} 4 \mathrm{H} 4 \mathrm{O}-\mathrm{HNCH}+\backslash \backslash 1,1 \backslash 0,0.98$ $19188939,-1.1257882394,0.3940697894 \backslash C, 0.3861789172,0.1107375342,0.9085$ $773873 \backslash \mathrm{C}, 1.1232235645,1.1732720436,0.2067601391 \backslash \mathrm{C}, 2.0223627245,0.60450$ $31145,-0.648471556 \backslash \mathrm{C}, 1.8766226372,-0.7985346626,-0.4769556376 \backslash \mathrm{H},-0.679$ $3137587,0.0676272611,0.6414340109 \backslash \mathrm{H}, 2.7117332716,1.0849983033,-1.32347$ $85818 \backslash \mathrm{H}, 2.3962257358,-1.6143166174,-0.9635567652 \backslash \mathrm{H}, 0.9380991838,2.2277$ $346713,0.3552218389 \backslash \mathrm{H}, 0.5084143718,0.086842437,1.9931288293 \backslash \mathrm{N},-2.65405$ $74229,0.0802601557,-0.1139025364 \backslash \mathrm{C},-3.795039595,0.015712515,-0.3920223$ $7 \backslash \mathrm{H},-4.8321974858,-0.0425444978,-0.6453176681 \backslash \backslash$ Version $=x 86-$ Linux $-\mathrm{G} 03 \mathrm{Re}$ vB. 03 $\backslash$ State $=1-A \backslash H F=-321.8686241 \backslash M P 2=-322.864484 \backslash R M S D=8.658 e-09 \backslash R M S F=2$. $155 e-05 \backslash$ Dipole $=0.7052288,0.3590432,-0.3024804 \backslash P G=C 01 \quad[X(C 5 H 6 N 101)] \backslash \backslash @$

\section{$\mathrm{C}_{4} \mathrm{H}_{4} \mathrm{O} \cdots \mathrm{H}_{-}-\mathrm{NCH}^{+}(\mathrm{C3})$}

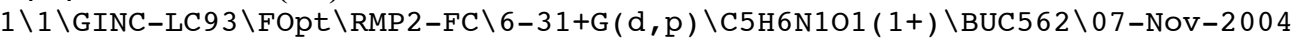
$\backslash 0 \backslash \backslash \#$ MP2/6-31+G(D,P) OPT MAXDISK $=1342177280 \backslash \backslash \mathrm{C} 4 \mathrm{H} 4 \mathrm{O}-\mathrm{HNCH}+\backslash \backslash 1,1 \backslash \mathrm{O}, 1.10$ $39188467,-0.7816986554,-0.6316605362 \backslash \mathrm{C}, 0.7060063004,-1.0708723144,0.64$ $14370527 \backslash \mathrm{C}, 0.6134744738,0.0934167239,1.379694162 \backslash \mathrm{C}, 1.0010921869,1.1577$ $268046,0.5049319404 \backslash \mathrm{C}, 1.2881602681,0.5749258314,-0.7020634147 \backslash \mathrm{H}, 1.0743$ $206684,2.2063526674,0.7412439087 \backslash \mathrm{H}, 1.6278195555,0.9499412079,-1.652304$ $1105 \backslash \mathrm{H}, 0.3665542359,0.1561477008,2.4283962149 \backslash \mathrm{H}, 0.5865930567,-2.115528$ $1733,0.8784288349 \backslash \mathrm{H},-0.9333834037,-0.1930565769,0.3395410775 \backslash \mathrm{N},-1.8135$ $876777,-0.0189254714,-0.2782876358 \backslash \mathrm{C},-2.8003528933,0.1037874812,-0.873$ $5939391 \backslash \mathrm{H},-3.7084231575,0.2283035575,-1.4364429924 \backslash \backslash$ Version $=x 86-\mathrm{Linux}-$ G03RevB.03 \State $=1-\mathrm{A} \backslash \mathrm{HF}=-321.8177749 \backslash \mathrm{MP} 2=-322.8397185 \backslash \mathrm{RMSD}=3.541 \mathrm{e}-09 \backslash \mathrm{R}$ $\mathrm{MSF}=5.845 \mathrm{e}-05 \backslash \mathrm{Dipole}=-2.9879636,0.2946279,-0.5208795 \backslash \mathrm{PG}=\mathrm{C} 01 \quad[\mathrm{X}(\mathrm{C} 5 \mathrm{H} 6 \mathrm{~N} 10$ $1)] \backslash \backslash @$

\section{$\mathrm{C}_{4} \mathrm{H}_{4} \mathrm{O} \cdots \cdot \mathrm{H}-\mathrm{CNH}^{+}(\mathrm{O1})$}

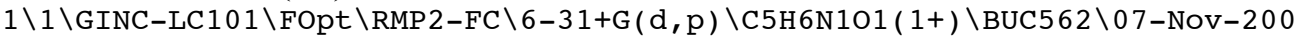
$4 \backslash 0 \backslash \backslash \#$ MP2 /6-31+G(D,P) OPT MAXDISK $=1342177280 \backslash \backslash \mathrm{C} 4 \mathrm{H} 4 \mathrm{O}-\mathrm{HCNH}+\backslash \backslash 1,1 \backslash \mathrm{O}, 0$. , $-0.3504602884,0.1517153076 \backslash \mathrm{C},-1.118487648,-0.1843848234,0.9656991094 \backslash \mathrm{C}$ $, 1.118487648,-0.1843848234,0.9656991094 \backslash C,-0.7168325946,0.0647601496,2$ $.2405750344 \backslash \mathrm{C}, 0.7168325946,0.0647601496,2.2405750344 \backslash \mathrm{H},-2.0733583574,-$ $0.3036415691,0.4840627973 \backslash \mathrm{H}, 2.0733583574,-0.3036415691,0.4840627973 \backslash \mathrm{H}$, $-1.3683514057,0.2204719684,3.0840954462 \backslash \mathrm{H}, 1.3683514057,0.2204719684,3$. $0840954462 \backslash \mathrm{H}, 0 .,-0.0267252269,-1.4043125993 \backslash \mathrm{C}, 0 ., 0.1812399753,-2.52263$ $49318 \backslash \mathrm{N}, 0 ., 0.3949344264,-3.6612105658 \backslash \mathrm{H}, 0.0 .5802619839,-4.6567325226 \backslash$ $\backslash$ Version=x86-Linux-G03RevB.03 \State $=1-A^{\prime} \backslash \mathrm{HF}=-321.8228603 \backslash \mathrm{MP} 2=-322.8325$ $85 \backslash \mathrm{RMSD}=6.258 \mathrm{e}-09 \backslash \mathrm{RMSF}=1.753 e-05 \backslash \mathrm{Dipole}=0 ., 0.516119,-4.0615679 \backslash \mathrm{PG}=\mathrm{CS}$ [ $\mathrm{SG}(\mathrm{C} 1 \mathrm{H} 2 \mathrm{~N} 1 \mathrm{O} 1), \mathrm{X}(\mathrm{C} 4 \mathrm{H} 4)] \backslash \backslash @$

\section{$\mathrm{C}_{4} \mathrm{H}_{4} \mathrm{O} \cdots \cdot \mathrm{H}-\mathrm{CNH}^{+}(\mathrm{C} 3)$}

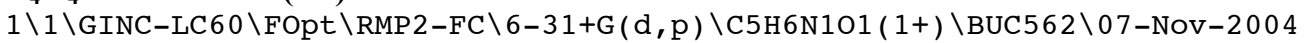
$\backslash 0 \backslash \backslash \#$ MP2 $/ 6-31+G(D, P)$ OPT MAXDISK $=1342177280 \backslash \backslash C 4 \mathrm{H} 4 \mathrm{O}-\mathrm{HCNH}+\backslash \backslash 1,1 \backslash 0,0.93$ $89344664,-0.8355180919,-0.574660464 \backslash C, 0.8436717478,-1.099386317,0.7660$ $441886 \backslash \mathrm{C}, 0.8443903592,0.081070368,1.4786217437 \backslash \mathrm{C}, 0.9499581783,1.131492$ $8179,0.5222206722 \backslash \mathrm{C}, 0.994337782,0.5281437309,-0.7130521633 \backslash \mathrm{H}, 1.0061357$ $968,2.1908786653,0.7134690202 \backslash \mathrm{H}, 1.131786997,0.8905146483,-1.7181548999$ $\backslash \mathrm{H}, 0.8103579368,0.1688574911,2.5529475107 \backslash \mathrm{H}, 0.8488483768,-2.1405967758$ $, 1.0433150783 \backslash \mathrm{H},-1.0161924979,-0.3684819867,0.4446559673 \backslash \mathrm{C},-1.73143546$ $82,-0.0170822011,-0.323456219 \backslash N,-2.6201862283,0.2421882622,-1.02221256$ $31 \backslash \mathrm{H},-3.3566443371,0.5022244651,-1.665730357 \backslash \backslash$ Version $=x 86-$ Linux-G03Rev B. 03 $\backslash$ State $=1-A \backslash H F=-321.8171597 \backslash M P 2=-322.8346445 \backslash R M S D=5.668 e-09 \backslash R M S F=3$. $162 e-05 \backslash \mathrm{Dipole}=-2.8011277,0.4515716,-0.6679766 \backslash \mathrm{PG}=\mathrm{C} 01 \quad[\mathrm{X}(\mathrm{C} 5 \mathrm{H} 6 \mathrm{~N} 101)] \backslash \backslash @$ 


\section{$\mathrm{C}_{4} \mathrm{H}_{4} \mathrm{O} \bullet \bullet \cdot \mathrm{NH}_{4}^{+}(\mathrm{O} 1)$}

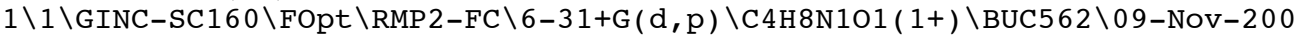
$4 \backslash 0 \backslash \backslash \#$ MP2 $/ 6-31+G(D, P) \quad O P T$ SYMMETRY=LOOSE MAXDISK=1342177280 \C4H4O $\mathrm{NH} 4+\backslash \backslash 1,1 \backslash 0,0 .,-0.2549414876,-0.2473361402 \backslash \mathrm{C}, 1.1151463872,-0.102013475$ $3,0.5702845885 \backslash \mathrm{C},-1.1151463872,-0.1020134753,0.5702845885 \backslash \mathrm{C}, 0.71632801$ $57,0.1334353469,1.8493417949 \backslash \mathrm{C},-0.7163280157,0.1334353469,1.8493417949$ $\backslash \mathrm{H}, 2.0718121362,-0.2130308517,0.0901810694 \backslash \mathrm{H},-2.0718121362,-0.21303085$ $17,0.0901810694 \backslash \mathrm{H}, 1.3687234247,0.2811434177,2.6935956533 \backslash \mathrm{H},-1.36872342$ $47,0.2811434177,2.6935956533 \backslash \mathrm{H}, 0 .,-0.0056466853,-1.925751397 \backslash \mathrm{N}, 0 ., 0.13$ $92050653,-2.9663366536 \backslash \mathrm{H}, 0.8298531735,-0.2899833607,-3.3774404018 \backslash \mathrm{H},-0$ $.8298531735,-0.2899833607,-3.3774404018 \backslash \mathrm{H}, 0 ., 1.1374222594,-3.179392148$ $5 \backslash \backslash$ Version=DEC-AXP-OSF $/ 1-G 03 R e v B .03 \backslash$ State $=1-A^{\prime} \backslash H F=-285.2042469 \backslash M P 2=-28$ $6.1157312 \backslash \mathrm{RMSD}=5.181 \mathrm{e}-09 \backslash \mathrm{RMSF}=3.247 \mathrm{e}-05 \backslash \mathrm{Dipole}=0,0.2975278,-4.1102383$ $\backslash \mathrm{PG}=\mathrm{CS} \quad[\mathrm{SG}(\mathrm{H} 2 \mathrm{~N} 1 \mathrm{O} 1), \mathrm{X}(\mathrm{C} 4 \mathrm{H} 6)] \backslash \backslash @$

\section{$\mathrm{C}_{4} \mathrm{H}_{4} \mathrm{O} \cdots \cdot \mathrm{NH}_{4}{ }^{+}(\mathrm{C} 3)$}

$1 \backslash 1 \backslash$ GINC-LC65 $\backslash$ FOpt $\backslash$ RMP $2-F C \backslash 6-31+G(d, p) \backslash C 4 H 8 N 101(1+) \backslash B U C 562 \backslash 08-N o v-2004$ $\backslash 0 \backslash \backslash \#$ MP2 $/ 6-31+G(D, P)$ OPT MAXDISK $=1342177280 \backslash \backslash C 4 \mathrm{H} 4 \mathrm{O}-\mathrm{NH} 4+\backslash \backslash 1,1 \backslash 0,-0.8$ $980166874,0.7416785063,-0.5296504141 \backslash \mathrm{C},-0.5838027964,-0.476698825,-1.0$ $750479379 \backslash \mathrm{C}, 0.7691668624,-0.6995057744,-0.9787979539 \backslash \mathrm{C}, 1.3277926352,0$. $4639378452,-0.3567668889 \backslash \mathrm{C}, 0.2794669614,1.3121791516,-0.110133315 \backslash \mathrm{H},-0$ $.0916545473,-1.0190743028,1.090810074 \backslash \mathrm{H}, 2.3682250311,0.6661518457,-0.1$ $60318398 \backslash \mathrm{H}, 0.2000695273,2.3106004442,0.2857787121 \backslash \mathrm{H}, 1.2989430916,-1.55$ $51216125,-1.3665691203 \backslash \mathrm{H},-1.4027668408,-1.0195173644,-1.5168594124 \backslash \mathrm{N},-$ $0.548425472,-0.9047485501,2.0135554525 \backslash \mathrm{H},-1.0188260582,-1.7707293503,2$ $.2801235375 \backslash \mathrm{H}, 0.1470640259,-0.6615226459,2.7197589759 \backslash \mathrm{H},-1.2336844024$, $-0.1504495989,1.9340673503 \backslash \backslash$ Version $=x 86-$ Linux-G03RevB.03 $\backslash$ State $=1-A \backslash H F=$ $-285.2003357 \backslash M P 2=-286.1154013 \backslash \mathrm{RMSD}=3.526 \mathrm{e}-09 \backslash \mathrm{RMSF}=1.148 \mathrm{e}-05 \backslash \mathrm{Dipole}=-0$. $4861693,-1.4509152,2.9266202 \backslash \mathrm{PG}=\mathrm{C} 01 \quad[\mathrm{X}(\mathrm{C} 4 \mathrm{H} 8 \mathrm{~N} 101)] \backslash \backslash @$

\section{$\mathrm{C}_{4} \mathrm{H}_{4} \mathrm{O} \cdots \mathrm{LiCNH}^{+}(\mathrm{O1})$}

$1 \backslash 1 \backslash$ GINC-LC15 $\backslash F O p t \backslash R M P 2-F C \backslash 6-31+G(d, p) \backslash C 5 H 5 L i 1 N 101(1+) \backslash$ BUC562 $\backslash 08-N o v-2$

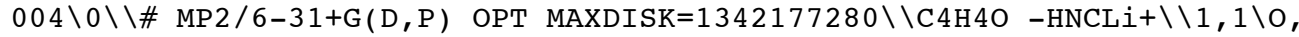
$0 .,-0.2196198423,0.3501665676 \backslash C,-1.114794194,-0.1431727381,1.172798788$ $6 \backslash \mathrm{C}, 1.114794194,-0.1431727381,1.1727987886 \backslash \mathrm{C},-0.7163548153,-0.02933793$ $36,2.4693237884 \backslash \mathrm{C}, 0.7163548153,-0.0293379336,2.4693237884 \backslash \mathrm{H},-2.0683544$ $819,-0.1964722169,0.6776678754 \backslash \mathrm{H}, 2.0683544819,-0.1964722169,0.67766787$ $54 \backslash \mathrm{H},-1.3688246554,0.0419308496,3.3232297003 \backslash \mathrm{H}, 1.3688246554,0.04193084$ $96,3.3232297003 \backslash \mathrm{H}, 0 .,-0.00957982,-1.2480914729 \backslash \mathrm{N}, 0 ., 0.1250805836,-2.28$ $6935022 \backslash \mathrm{C}, 0.0 .2759650494,-3.4518309988 \backslash \mathrm{Li}, 0 ., 0.5381316574,-5.51365866$ $57 \backslash \backslash$ Version $=x 86-$ Linux-G03RevB. 03 $\backslash$ State $=1-A^{\prime} \backslash \mathrm{HF}=-328.8048977 \backslash \mathrm{MP} 2=-329.8$ $147664 \backslash \mathrm{RMSD}=5.290 \mathrm{e}-09 \backslash \mathrm{RMSF}=5.043 \mathrm{e}-06 \backslash \mathrm{Dipole}=0,0.7149402,-6.8521982 \backslash \mathrm{PG}$ $=\mathrm{CS}[\mathrm{SG}(\mathrm{C} 1 \mathrm{H} 1 \mathrm{Li} 1 \mathrm{~N} 1 \mathrm{O} 1), \mathrm{X}(\mathrm{C} 4 \mathrm{H} 4)] \backslash \backslash @$

\section{$\mathrm{C}_{4} \mathrm{H}_{4} \mathrm{O} \cdots \mathrm{LiCNH}^{+}(\mathrm{C} 3)$}

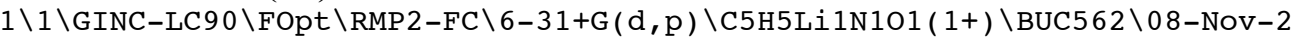

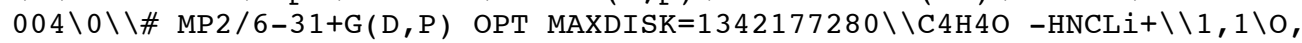
$1.3251985814,-0.8040950655,-0.5522453757 \backslash C, 0.9974440958,-1.1006690232$, $0.7413406661 \backslash \mathrm{C}, 0.9237249684,0.0539955733,1.485581693 \backslash \mathrm{C}, 1.2434676136,1$. $1280832029,0.5940013843 \backslash \mathrm{C}, 1.4811925561,0.5548794349,-0.6280993087 \backslash \mathrm{H}, 1$. $3133966122,2.177251946,0.8291392684 \backslash \mathrm{H}, 1.7700356581,0.9388570393,-1.590$ $9304703 \backslash \mathrm{H}, 0.7219937548,0.1137258209,2.5432194742 \backslash \mathrm{H}, 0.8869982754,-2.146$ $6257753,0.9717403219 \backslash \mathrm{H},-0.8748608447,0.0044350099,0.3501292961 \backslash \mathrm{N},-1.74$ $5046151,0.0589696189,-0.1955943261 \backslash C,-2.7479330558,0.0993403679,-0.805$ $6246499 \backslash \mathrm{Li},-4.5304020395,0.1728506056,-1.8797911035 \backslash \backslash$ Version=x86-Linux - G03RevB.03 \State $=1-A \backslash H F=-328.7966485 \backslash M P 2=-329.8112209 \backslash \mathrm{RMSD}=8.763 \mathrm{e}-09 \backslash$ $\mathrm{RMSF}=4.484 \mathrm{e}-05 \backslash \mathrm{Dipole}=-5.9576814,0.4236217,-2.0761989 \backslash \mathrm{PG}=\mathrm{C} 01 \quad[\mathrm{X}(\mathrm{C} 5 \mathrm{H} 5 \mathrm{Li}$ $1 \mathrm{~N} 101)] \backslash \backslash @$ 


\section{$\mathrm{C}_{4} \mathrm{H}_{4} \mathrm{O} \cdots \mathrm{NaCNH}^{+}(\mathrm{O1})$}

$1 \backslash 1 \backslash G I N C-L C 52 \backslash F O p t \backslash R M P 2-F C \backslash 6-31+G(d, p) \backslash C 5 H 5 N 1 N a 101(1+) \backslash B U C 562 \backslash 09-N o v-2$ $004 \backslash 0 \backslash \backslash \#$ MP2 $/ 6-31+G(D, P) \quad O P T$ SYMMETRY=LOOSE MAXDISK $=1342177280 \backslash \backslash \mathrm{C} 4 \mathrm{H} 4 \mathrm{O}$

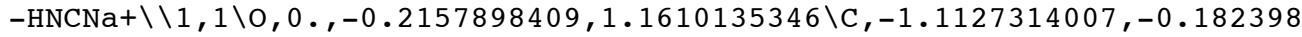
$503,1.9854739256 \backslash \mathrm{C}, 1.1127314007,-0.182398503,1.9854739256 \backslash \mathrm{C},-0.7161042$ $801,-0.133295677,3.2874034646 \backslash C, 0.7161042801,-0.133295677,3.2874034646$ $\backslash \mathrm{H},-2.0663633985,-0.2068469028,1.4883208235 \backslash \mathrm{H}, 2.0663633985,-0.20684690$ $28,1.4883208235 \backslash \mathrm{H},-1.3690530985,-0.1032719088,4.1433663212 \backslash \mathrm{H}, 1.3690530$ $985,-0.1032719088,4.1433663212 \backslash \mathrm{H}, 0 .,-0.0427538317,-0.4948628152 \backslash \mathrm{N}, 0 ., 0$ $.0622047507,-1.5274163098 \backslash \mathrm{C}, 0 ., 0.1810533829,-2.698399888 \backslash \mathrm{Na}, 0 ., 0.42326$ $24354,-5.1317122671 \backslash \backslash$ Version=x86-Linux-G03RevB. 03 $\backslash$ State $=1-A^{\prime} \backslash \mathrm{HF}=-483.2$ $120875 \backslash \mathrm{MP} 2=-484.2204577 \backslash \mathrm{RMSD}=8.648 \mathrm{e}-09 \backslash \mathrm{RMSF}=8.363 \mathrm{e}-05 \backslash \mathrm{Dipole}=0 ., 0.5605$ $344,-6.3994374 \backslash \mathrm{PG}=\mathrm{CS}[\mathrm{SG}(\mathrm{C} 1 \mathrm{H} 1 \mathrm{~N} 1 \mathrm{Na} 1 \mathrm{O} 1), \mathrm{X}(\mathrm{C} 4 \mathrm{H} 4)] \backslash \backslash @$

\section{$\mathrm{C}_{4} \mathrm{H}_{4} \mathrm{O} \cdots \mathrm{NaCNH}^{+}(\mathrm{C} 3)$}

$1 \backslash 1 \backslash$ GINC-LC1 48 $\backslash$ FOpt $\backslash$ RMP2-FC \6-31+G (d,p) \C5H5N1Na101 ( 1+) \BUC562 \09-Nov$2004 \backslash 0 \backslash \backslash \#$ MP2 $/ 6-31+G(D, P) \quad O P T$ MAXDISK $=1342177280 \backslash \backslash \mathrm{C} 4 \mathrm{H} 4 \mathrm{O}-\mathrm{HNCNa}+\backslash \backslash 1,1 \backslash \mathrm{O}$ $, 1.9877528707,-0.8264573866,-0.2783009788 \backslash \mathrm{C}, 1.6699327087,-1.1244035715$ $, 1.0176989519 \backslash \mathrm{C}, 1.6051631106,0.0280784506,1.7646132149 \backslash \mathrm{C}, 1.9186661505$, $1.1033070892,0.8724007636 \backslash \mathrm{C}, 2.1452303396,0.5321941556,-0.3527014511 \backslash \mathrm{H}$, $1.9916668951,2.1519756379,1.1087560179 \backslash \mathrm{H}, 2.4243129642,0.9180256888,-1$. $3175835104 \backslash \mathrm{H}, 1.4098192057,0.0865352891,2.8233896713 \backslash \mathrm{H}, 1.5551311627,-2$. $170186227,1.246051921 \backslash \mathrm{H},-0.2412737402,0.0131230163,0.6135761073 \backslash \mathrm{N},-1.1$ $150020814,0.0549462469,0.0829151986 \backslash \mathrm{C},-2.1259376212,0.0838963532,-0.51$ $91564923 \backslash \mathrm{Na},-4.2286357284,0.1353751906,-1.7750289718 \backslash \backslash$ Version=x86-Linu $\mathrm{X}-\mathrm{G} 03$ RevB.03 $\backslash$ State $=1-\mathrm{A} \backslash \mathrm{HF}=-483.2042894 \backslash \mathrm{MP} 2=-484.2172396 \backslash \mathrm{RMSD}=5.010 \mathrm{e}-09$ $\backslash \mathrm{RMSF}=3.904 \mathrm{e}-05 \backslash \mathrm{Dipole}=-5.5866389,0.3753423,-1.9926135 \backslash \mathrm{PG}=\mathrm{C} 01 \quad[\mathrm{X}(\mathrm{C} 5 \mathrm{H} 5 \mathrm{~N}$ $1 \mathrm{Na1O1})] \backslash \backslash @$

\section{$\mathrm{C}_{4} \mathrm{H}_{4} \mathrm{O} \cdots \mathrm{NaNCH}^{+}(\mathrm{O1})$}

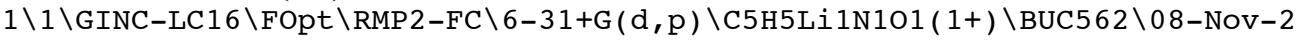

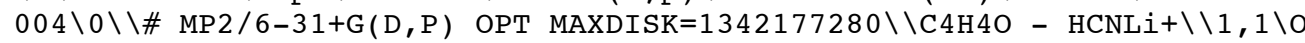
$, 0 .,-0.4168665933,0.4657987694 \backslash \mathrm{C},-1.1088647432,-0.2305966669,1.2706788$ $47 \backslash C, 1.1088647432,-0.2305966669,1.270678847 \backslash C,-0.7156411403,0.06105489$ $77,2.5431334927 \backslash \mathrm{C}, 0.7156411403,0.0610548977,2.5431334927 \backslash \mathrm{H},-2.06397924$ $97,-0.3602221807,0.7924512433 \backslash \mathrm{H}, 2.0639792497,-0.3602221807,0.792451243$ $3 \backslash \mathrm{H},-1.3696479163,0.2452960541,3.3788498909 \backslash \mathrm{H}, 1.3696479163,0.245296054$ $1,3.3788498909 \backslash \mathrm{H}, 0 .,-0.0502026166,-1.3788285074 \backslash \mathrm{C}, 0 ., 0.1510405635,-2.4$ $489648147 \backslash \mathrm{N}, 0.0 .3655378433,-3.6016901563 \backslash \mathrm{Li}, 0 ., 0.7281601873,-5.516764$ $0034 \backslash \backslash$ Version $=x 86-\mathrm{L}$ inux-G03RevB.03 $\backslash$ State $=1-A^{\prime} \backslash \mathrm{HF}=-328.8219612 \backslash \mathrm{MP} 2=-329$ $.8349697 \backslash \mathrm{RMSD}=4.427 \mathrm{e}-09 \backslash \mathrm{RMSF}=4.616 \mathrm{e}-05 \backslash \mathrm{Dipole}=0,1.0362172,-7.3164527 \backslash$ $\mathrm{PG}=\mathrm{CS}[\mathrm{SG}(\mathrm{C} 1 \mathrm{H} 1 \mathrm{Li} 1 \mathrm{~N} 1 \mathrm{O} 1), \mathrm{X}(\mathrm{C} 4 \mathrm{H} 4)] \backslash \backslash @$

\section{$\mathrm{C}_{4} \mathrm{H}_{4} \mathrm{O} \cdots \mathrm{NaNCH}^{+}(\mathrm{C} 3)$}

$1 \backslash 1 \backslash$ GINC-LC15 $\backslash$ FOpt $\backslash$ RMP 2-FC $\backslash 6-31+G(d, p) \backslash C 5 H 5 L i 1 N 1 O 1(1+) \backslash B U C 562 \backslash 09-N o v-2$

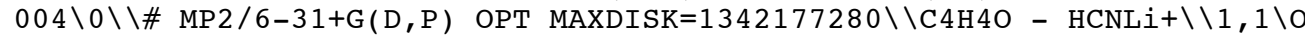
$,-0.2829053235,-1.3149422406,0.7367074617 \backslash C, 0.6866151199,-1.5776080924$ $,-0.1952523136 \backslash \mathrm{C}, 0.2358667459,-1.2497545937,-1.4504268927 \backslash \mathrm{C},-1.1000514$ $023,-0.7669058397,-1.2857571276 \backslash C,-1.3699143424,-0.8279664258,0.057105$ $2419 \backslash \mathrm{H}, 0.6416412348,0.7360862562,-0.2512770338 \backslash \mathrm{H},-1.7810211416,-0.4442$ $954167,-2.0561474826 \backslash \mathrm{H},-2.2373730865,-0.6120755691,0.6564970204 \backslash \mathrm{H}, 0.77$ $95531202,-1.3790708986,-2.3722767072 \backslash \mathrm{H}, 1.5951420741,-2.0142011785,0.18$ $30211489 \backslash \mathrm{C}, 0.7004920341,1.6353041611,0.3454218368 \backslash \mathrm{N}, 0.7857788216,2.610$ $9196522,0.989976506 \backslash \mathrm{Li}, 0.9489332351,4.2260806363,2.0633811167 \backslash \backslash$ Version $=x 86-$ Linux-G03RevB.03 $\backslash$ State $=1-A \backslash H F=-328.8180775 \backslash M P 2=-329.8357455 \backslash$ RMSD $=$ $8.476 \mathrm{e}-09 \backslash \mathrm{RMSF}=1.638 \mathrm{e}-05 \backslash \mathrm{Dipole}=1.3655649,5.6768961,2.277427 \backslash \mathrm{PG}=\mathrm{C} 01 \quad[\mathrm{X}$ (C5H5Li1N1O1) ] \\@

\section{$\mathrm{C}_{4} \mathrm{H}_{4} \mathrm{O} \bullet \bullet \cdot \mathrm{HBr}(\mathrm{O1})$}

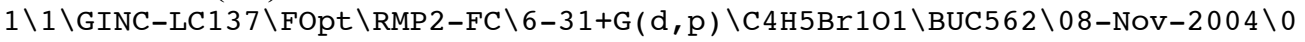
$\backslash \backslash \#$ MP2/6-31+G(D,P) OPT SYMMETRY=LOOSE MAXDISK=1342177280 \C4H4O - HBr $\backslash \backslash 0,1 \backslash 0,0 ., 0 .,-1.1011070118 \backslash \mathrm{C}, 0 ., 1.1035363767,-1.9180421485 \backslash \mathrm{C}, 0 ., 0.715$ $23007,-3.2283078593 \backslash \mathrm{C}, 0 .,-1.1035363767,-1.9180421485 \backslash \mathrm{C}, 0 .,-0.71523007$, $-3.2283078593 \backslash \mathrm{H}, 0.2 .0553432555,-1.416744751 \backslash \mathrm{H}, 0 ., 1.3716167841,-4.0823$ $350093 \backslash \mathrm{H}, 0 .,-1.3716167841,-4.0823350093 \backslash \mathrm{H}, 0 .,-2.0553432555,-1.41674475$ $1 \backslash \mathrm{H}, 0 ., 0 ., 0.8888316432 \backslash \mathrm{Br}, 0 ., 0 ., 2.3049824019 \backslash \backslash$ Version=x86-Linux-G03Rev B. 03 \State $=1-\mathrm{A} 1 \backslash \mathrm{HF}=-2799.1274131 \backslash \mathrm{MP} 2=-2799.9717571 \backslash \mathrm{RMSD}=3.428 \mathrm{e}-09 \backslash \mathrm{RMSF}$ $=6.873 e-05 \backslash \mathrm{Dipole}=0 ., 0 .,-1.1129433 \backslash \mathrm{PG}=\mathrm{C} 02 \mathrm{~V} \quad[\mathrm{C} 2(\mathrm{O} 1 \mathrm{H} 1 \mathrm{Br} 1), \mathrm{SGV}(\mathrm{C} 4 \mathrm{H} 4)] \backslash \backslash @$ 


\section{$\mathrm{C}_{4} \mathrm{H}_{4} \mathrm{O} \cdots \cdot \mathrm{HBr}(\mathrm{C3})$}

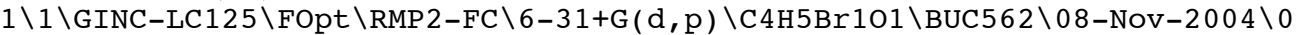

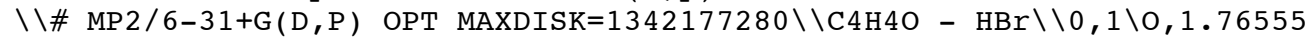
$04714,-0.8902922864,0.0540440148 \backslash \mathrm{C}, 1.4932021187,-0.5645870581,1.353466$ $1293 \backslash C, 1.500810971,0.7978144384,1.5119579198 \backslash C, 1.8005927401,1.34910040$ $05,0.2268919398 \backslash \mathrm{C}, 1.9496001862,0.2846873124,-0.621952988 \backslash \mathrm{H}, 1.888404179$ $, 2.3896171928,-0.0379121509 \backslash \mathrm{H}, 2.1624336647,0.1900027312,-1.6719925695 \backslash$ $\mathrm{H}, 1.3283511025,1.3302640347,2.4328651517 \backslash \mathrm{H}, 1.3296424632,-1.3937273101$, $2.0193296904 \backslash \mathrm{H},-0.6680918527,0.2531487598,0.2936261558 \backslash \mathrm{Br},-1.732296555$ $,-0.1956873622,-0.5225841829 \backslash \backslash$ Version=x86-Linux-G03RevB.03 $\backslash$ State $=1-A \backslash H$ $\mathrm{F}=-2799.124137 \backslash \mathrm{MP} 2=-2799.9718595 \backslash \mathrm{RMSD}=8.966 \mathrm{e}-09 \backslash \mathrm{RMSF}=8.438 \mathrm{e}-05 \backslash \mathrm{Dipole}=$ $0.5267623,0.3904929,0.3615914 \backslash \mathrm{PG}=\mathrm{C} 01 \quad[\mathrm{X}(\mathrm{C} 4 \mathrm{H} 5 \mathrm{Br} 1 \mathrm{O} 1)] \backslash \backslash \mathrm{C}$

\section{$\mathrm{C}_{4} \mathrm{H}_{4} \mathrm{O} \cdots \cdot \mathrm{HCl}(\mathrm{O1})$}

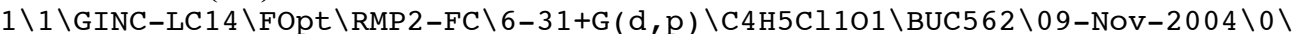
$\backslash \#$ MP2 $/ 6-31+G(D, P)$ OPT SYMMETRY=LOOSE MAXDISK $=1342177280 \backslash \backslash C 4 \mathrm{H} 4 \mathrm{O}-\mathrm{HCl} \backslash$ $\backslash 0,1 \backslash 0,0 ., 0 \ldots,-0.2823353705 \backslash \mathrm{C}, 0 ., 1.1038968753,-1.099260823 \backslash \mathrm{C}, 0,0.71527$ $72887,-2.4091638378 \backslash \mathrm{C}, 0 .,-1.1038968753,-1.099260823 \backslash \mathrm{C}, 0,,-0.7152772887$ $,-2.4091638378 \backslash \mathrm{H}, 0 ., 2.0556880445,-0.5980236612 \backslash \mathrm{H}, 0 ., 1.3714816205,-3.26$ $32915052 \backslash \mathrm{H}, 0 .,-1.3714816205,-3.2632915052 \backslash \mathrm{H}, 0 .,-2.0556880445,-0.598023$ $6612 \backslash \mathrm{H}, 0 ., 0 ., 1.6850124715 \backslash \mathrm{Cl}, 0 ., 0 ., 2.9645527503 \backslash \backslash$ Version $=x 86-\mathrm{Linux}-\mathrm{G} 03$ RevB.03 $\backslash$ State $=1-\mathrm{A} 1 \backslash \mathrm{HF}=-688.7098411 \backslash \mathrm{MP} 2=-689.5723012 \backslash \mathrm{RMSD}=4.010 \mathrm{e}-09 \backslash \mathrm{RMS}$ $\mathrm{F}=1.722 \mathrm{e}-05 \backslash \mathrm{Dipole}=0,0 .,-1.2175911 \backslash \mathrm{PG}=\mathrm{C} 02 \mathrm{~V}[\mathrm{C} 2(\mathrm{O} 1 \mathrm{H} 1 \mathrm{Cl} 1), \mathrm{SGV}(\mathrm{C} 4 \mathrm{H} 4)] \backslash \backslash @$

\section{$\mathrm{C}_{4} \mathrm{H}_{4} \mathrm{O} \cdots \cdot \mathrm{HCl}(\mathrm{C3})$}

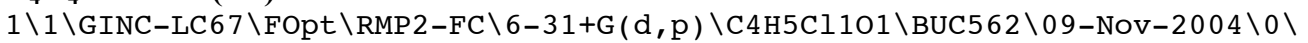

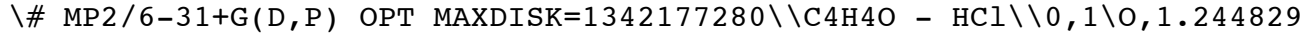
$648,-1.0024204824,-0.1726919257 \backslash C, 0.9094481997,-0.6794834627,1.1124137$ $994 \backslash \mathrm{C}, 0.8569990803,0.6829407426,1.260297178 \backslash \mathrm{C}, 1.1881884092,1.237641360$ $2,-0.0156600165 \backslash \mathrm{C}, 1.4145438609,0.1746711468,-0.848686964 \backslash \mathrm{H}, 1.247226848$ $5,2.2790073566,-0.2847539328 \backslash \mathrm{H}, 1.6762278861,0.0828879398,-1.8878785495$ $\backslash \mathrm{H}, 0.6259036578,1.2135959069,2.1693021822 \backslash \mathrm{H}, 0.7470872418,-1.5096775016$ $, 1.7772234557 \backslash \mathrm{H},-1.2450413002,0.1365381497,0.0833435724 \backslash \mathrm{Cl},-2.30735993$ $05,-0.1575062773,-0.5603461943 \backslash \backslash$ Version $=x 86-L$ inux-G03RevB.03 $\backslash$ State $=1-A$ $\backslash \mathrm{HF}=-688.7059544 \backslash \mathrm{MP} 2=-689.571575 \backslash \mathrm{RMSD}=5.763 \mathrm{e}-09 \backslash \mathrm{RMSF}=4.166 \mathrm{e}-05 \backslash \mathrm{Dipole}=$ $0.6415079,0.3841063,0.3846746 \backslash \mathrm{PG}=\mathrm{C} 01 \quad[\mathrm{X}(\mathrm{C} 4 \mathrm{H} 5 \mathrm{Cl} 1 \mathrm{O} 1)] \backslash \backslash @$

\section{$\mathrm{C}_{4} \mathrm{H}_{4} \mathrm{O} \cdots \mathrm{HNC}(\mathrm{O1})$}

$1 \backslash 1 \backslash$ GINC-SC59 \FOpt \RMP2-FC $66-31+\mathrm{G}(\mathrm{d}, \mathrm{p}) \backslash \mathrm{C} 5 \mathrm{H} 5 \mathrm{~N} 101 \backslash \mathrm{BUC} 562 \backslash 11-\mathrm{Nov}-2004 \backslash 0 \backslash \backslash$ \# MP2 /6-31+G(D,P) OPT MAXDISK $=1342177280 \backslash \backslash \mathrm{C} 4 \mathrm{H} 4 \mathrm{O}-\mathrm{HNC} \backslash \backslash 0,1 \backslash \mathrm{O}, 0 .,-0.105$ $4297308,-0.0992943691 \backslash \mathrm{C}, 1.105868495,-0.0078264493,-0.9124065918 \backslash \mathrm{C},-1.1$ $05868495,-0.0078264493,-0.9124065918 \backslash \mathrm{C}, 0.7154404109,0.1461730589,-2.21$ $15459399 \backslash \mathrm{C},-0.7154404109,0.1461730589,-2.2115459399 \backslash \mathrm{H}, 2.0580192922,-0$. $0672135383,-0.4153514797 \backslash \mathrm{H},-2.0580192922,-0.0672135383,-0.4153514797 \backslash \mathrm{H}$ $, 1.3710292817,0.2462843252,-3.0601734305 \backslash \mathrm{H},-1.3710292817,0.2462843252$, $-3.0601734305 \backslash \mathrm{H}, 0 .,-0.1042776293,1.7838821806 \backslash \mathrm{N}, 0 .,-0.0901675307,2.794$ $8196542 \backslash C, 0 .,-0.0732354498,3.9808692324 \backslash \backslash$ Version=DEC-AXP-OSF / 1-G03RevB $.03 \backslash$ State $=1-A^{\prime} \backslash \mathrm{HF}=-321.5075344 \backslash \mathrm{MP} 2=-322.5102365 \backslash \mathrm{RMSD}=4.060 \mathrm{e}-09 \backslash \mathrm{RMSF}=3$. $369 e-05 \backslash$ Dipole $=0,0.0372274,-2.0616719 \backslash \mathrm{PG}=\mathrm{CS} \quad[\mathrm{SG}(\mathrm{C} 1 \mathrm{H} 1 \mathrm{~N} 101), \mathrm{X}(\mathrm{C} 4 \mathrm{H} 4)] \backslash \backslash @$

\section{$\mathrm{C}_{4} \mathrm{H}_{4} \mathrm{O} \cdots \cdot \mathrm{HNC}(\mathrm{C3})$}

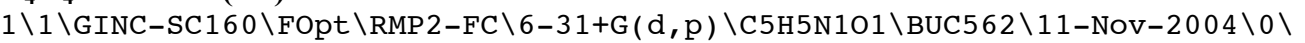
$\backslash \#$ MP2 /6-31+G(D,P) OPT MAXDISK $=1342177280 \backslash \backslash C 4 \mathrm{H} 4 \mathrm{O}-\mathrm{HNC} \backslash \backslash 0,1 \backslash 0,0.798572$ $3273,0.1598769016,-1.2947592213 \backslash \mathrm{C}, 1.0293557059,-0.9711022923,-0.561976$ $4809 \backslash \mathrm{C}, 1.2185833638,-0.6494122019,0.7582671469 \backslash \mathrm{C}, 1.1024619718,0.774306$ $2916,0.8447263736 \backslash \mathrm{C}, 0.8486170604,1.2165844305,-0.427095024 \backslash \mathrm{H},-1.131137$ $0097,-0.3325862828,0.5277372506 \backslash \mathrm{H}, 1.1966387033,1.3896348013,1.72401220$ $84 \backslash \mathrm{H}, 0.6804321585,2.1862072286,-0.8613788245 \backslash \mathrm{H}, 1.4310038226,-1.3424440$ $977,1.5557697607 \backslash \mathrm{H}, 1.0295081025,-1.8949520943,-1.1134194012 \backslash \mathrm{N},-2.13156$ $82828,-0.291649289,0.426975134 \backslash C,-3.3113591712,-0.2442645186,0.3088324$ $576 \backslash \backslash$ Version $=$ DEC-AXP-OSF $/ 1-G 03 R e v B .03 \backslash$ State $=1-A \backslash H F=-321.5021299 \backslash \mathrm{MP} 2=-3$ $22.508428 \backslash \mathrm{RMSD}=4.371 \mathrm{e}-09 \backslash \mathrm{RMSF}=1.955 \mathrm{e}-06 \backslash \mathrm{Dipole}=1.6445041,0.0021565,0.3$ $16736 \backslash \mathrm{PG}=\mathrm{C} 01 \quad[\mathrm{X}(\mathrm{C} 5 \mathrm{H} 5 \mathrm{~N} 1 \mathrm{O} 1)] \backslash \backslash @$ 


\section{$\mathrm{C}_{4} \mathrm{H}_{4} \mathrm{O} \cdots \cdot \mathrm{HCN}(\mathrm{O1})$}

$1 \backslash 1 \backslash$ GINC-SC65 $\backslash$ FOpt \RMP2-FC $\backslash 6-31+G(d, p) \backslash C 5 H 5 N 101 \backslash B U C 562 \backslash 11-N o v-2004 \backslash 0 \backslash \backslash$ \# MP2/6-31+G(D,P) OPT MAXDISK $=1342177280 \backslash \backslash \mathrm{C} 4 \mathrm{H} 4 \mathrm{O}-\mathrm{HCN} \backslash \backslash 0,1 \backslash 0,0,,-0.381$ $1178389,-0.227708935 \backslash \mathrm{C}, 1.102851325,-0.1428115274,-1.0106009668 \backslash \mathrm{C},-1.10$ $2851325,-0.1428115274,-1.0106009668 \backslash \mathrm{C}, 0.7150877088,0.2376889312,-2.264$ $7354791 \backslash \mathrm{C},-0.7150877088,0.2376889312,-2.2647354791 \backslash \mathrm{H}, 2.0560374601,-0.2$ $897711387,-0.5338437166 \backslash \mathrm{H},-2.0560374601,-0.2897711387,-0.5338437166 \backslash \mathrm{H}$, $1.3716892143,0.4834483778,-3.0824155574 \backslash \mathrm{H},-1.3716892143,0.4834483778,-$ $3.0824155574 \backslash \mathrm{H}, 0 .,-0.0922422812,1.8826014709 \backslash \mathrm{C}, 0 ., 0.0436639825,2.94532$ $85478 \backslash \mathrm{N}, 0 ., 0.1933311106,4.1148078029 \backslash \backslash$ Version=DEC-AXP-OSF / 1-G03RevB.03 $\backslash$ State $=1-A^{\prime} \backslash H F=-321.5178959 \backslash M P 2=-322.5351453 \backslash R M S D=6.911 e-09 \backslash R M S F=1.343$ e-04\Dipole $=0 .,-0.0610615,-1.7906959 \backslash P G=C S \quad[S G(C 1 H 1 N 101), X(C 4 H 4)] \backslash \backslash @$

\section{$\mathrm{C}_{4} \mathrm{H}_{4} \mathrm{O} \cdots \mathrm{HCN}(\mathrm{C3})$}

$1 \backslash 1 \backslash$ GINC-SC57 $\backslash$ FOpt \RMP2-FC $\backslash 6-31+\mathrm{G}(\mathrm{d}, \mathrm{p}) \backslash \mathrm{C} 5 \mathrm{H} 5 \mathrm{~N} 101 \backslash \mathrm{BUC} 562 \backslash 12-\mathrm{Nov}-2004 \backslash 0 \backslash \backslash$ \# MP2/6-31+G(D,P) OPT MAXDISK $=1342177280 \backslash \backslash C 4 \mathrm{H} 4 \mathrm{O}-\mathrm{HCN} \backslash \backslash 0,1 \backslash \mathrm{O}, 0.7737284$ $573,0.1179532033,-1.2399391634 \backslash C, 1.1069070806,-0.9984100525,-0.5216124$ $72 \backslash \mathrm{C}, 1.385973096,-0.6600000101,0.77731748 \backslash \mathrm{C}, 1.2191454666,0.7570866835$, $0.8677632376 \backslash \mathrm{C}, 0.8489185153,1.1813624896,-0.3810976806 \backslash \mathrm{H},-1.1748697489$ $,-0.3792293814,0.4730298068 \backslash \mathrm{H}, 1.3577309728,1.3815570166,1.7346691389 \backslash \mathrm{H}$ $, 0.6116178612,2.1413038652,-0.8048239533 \backslash \mathrm{H}, 1.6826210754,-1.3391794675$, $1.5595702506 \backslash \mathrm{H}, 1.1062434087,-1.9243716812,-1.0696415158 \backslash \mathrm{C},-2.230190278$ $8,-0.2630001497,0.3478297002 \backslash \mathrm{N},-3.393956358,-0.132277106,0.212215427 \backslash \backslash$ Version=DEC-AXP-OSF / 1-G03RevB.03 \State $=1-A \backslash H F=-321.5149593 \backslash \mathrm{MP} 2=-322.53$ $55944 \backslash \mathrm{RMSD}=5.955 \mathrm{e}-09 \backslash \mathrm{RMSF}=4.987 \mathrm{e}-05 \backslash \mathrm{Dipole}=1.5108106,-0.0863361,0.3649$ $832 \backslash \mathrm{PG}=\mathrm{C} 01 \quad[\mathrm{X}(\mathrm{C} 5 \mathrm{H} 5 \mathrm{~N} 1 \mathrm{O} 1)] \backslash \backslash @$ 
Table S2. MP2/aug'-cc-pVTZ Energies (hartrees) for the Neutral and Protonated Substrates

\begin{tabular}{lrrrcc}
\hline Substrate & Neutral & \multicolumn{5}{c}{ Protonated } \\
\cline { 3 - 6 } & & 1 & 2 & 3 & 5 \\
\hline $\mathrm{C}_{3} \mathrm{H}_{3}^{-}$ & -115.67872 & -116.35795 & & & \\
$\mathrm{C}_{4} \mathrm{H}_{4}$ & -154.33718 & -154.70913 & & & \\
$\mathrm{NH}_{2} \mathrm{CHNH}$ & -149.73497 & -150.05639 & & -150.10474 & \\
$\mathrm{C}_{2} \mathrm{H}_{2} \mathrm{NH}$ & -132.36381 & -132.72623 & -132.73058 & & \\
$\mathrm{NH}_{2} \mathrm{CHCH}$ & -133.67473 & -134.01177 & & -134.03341 & \\
$\mathrm{C}_{3} \mathrm{H}_{3} \mathrm{~N}$ & -170.38128 & -170.72334 & -170.71534 & -170.72383 & \\
$\mathrm{C}_{4} \mathrm{H}_{4} \mathrm{NH}$ & -209.74604 & -210.04986 & -210.07814 & -210.07025 & \\
$\mathrm{NH}_{2} \mathrm{CHO}$ & -169.61923 & -169.91769 & & -169.94437 & \\
$\mathrm{HOCHCHCHO}$ & -266.70157 & & & -267.00436 & -267.01974 \\
$\mathrm{C}_{4} \mathrm{H}_{4} \mathrm{O}$ & -229.59089 & -229.85833 & -229.90196 & -229.88285 & \\
$\mathrm{C}_{4} \mathrm{H}_{6}$ & -155.63616 & -155.94148 & & & \\
$\mathrm{HOCHO}$ & -189.48563 & -189.74373 & & -189.77524 & \\
\hline
\end{tabular}

Table S3. MP2/aug'-cc-pVTZ Energies (hartrees) for the Hydrogen-Bonded Complexes with $\mathrm{HF}$

\begin{tabular}{lcccc}
\hline Substrate & \multicolumn{4}{c}{ Hydrogen-bonded (HF) } \\
\cline { 2 - 5 } & \multicolumn{1}{c}{1} & 2 & 3 & 5 \\
\hline $\mathrm{C}_{3} \mathrm{H}_{3}{ }^{-}$ & -216.11474 & -216.04443 & & \\
$\mathrm{C}_{4} \mathrm{H}_{4}$ & -254.68629 & & & \\
$\mathrm{NH}_{2} \mathrm{CHNH}$ & -250.08826 & & -250.10101 & \\
$\mathrm{C}_{2} \mathrm{H}_{2} \mathrm{NH}$ & -232.72727 & -232.71054 & & \\
$\mathrm{NH}_{2} \mathrm{CHCH}$ & -234.03157 & & -234.02685 & \\
$\mathrm{C}_{3} \mathrm{H}_{3} \mathrm{~N}$ & -270.74056 & & -270.72356 & \\
$\mathrm{C}_{4} \mathrm{H}_{4} \mathrm{NH}$ & & & -310.09739 & \\
$\mathrm{NH}_{2} \mathrm{CHO}$ & -269.96785 & & -269.98058 & \\
$\mathrm{HOCHCHCHO}$ & -367.05269 & & -367.04708 & -367.05685 \\
$\mathrm{C}_{4} \mathrm{H}_{4} \mathrm{O}$ & -329.94114 & & -329.93911 & \\
$\mathrm{C}_{4} \mathrm{H}_{6}$ & -255.98449 & & & \\
$\mathrm{HOCHO}$ & -289.83428 & & -289.84321 & \\
\hline
\end{tabular}


Table S4. MP2/aug'-cc-pVTZ Energies (hartrees) for $\mathrm{A}-\mathrm{H}^{\mathrm{n}+}$ and $\mathrm{A}^{(\mathrm{n}-1)-}$, and the Hydrogen-Bonded Complexes of Vinylamine with $\mathrm{A}-\mathrm{H}^{\mathrm{n}+}$

\begin{tabular}{|c|c|c|c|c|}
\hline \multirow[t]{2}{*}{$\mathrm{A}-\mathrm{H}^{\mathrm{n}+}$} & \multirow[b]{2}{*}{$\mathrm{A}-\mathrm{H}^{\mathrm{n}+}$} & \multirow[b]{2}{*}{$\mathrm{A}^{(\mathrm{n}-1)+}$} & \multicolumn{2}{|c|}{$\mathrm{A}-\mathrm{H}^{\mathrm{n+}}$-vinylamine complex } \\
\hline & & & 1 & 3 \\
\hline $\mathrm{HF}-\mathrm{H}^{+}$ & -100.53207 & -100.34038 & -234.37017 & -234.39105 \\
\hline $\mathrm{HCl}-\mathrm{H}^{+}$ & -460.53200 & -460.31445 & -594.33908 & -594.35964 \\
\hline $\mathrm{HBr}-\mathrm{H}^{+}$ & -2573.52060 & -2573.29360 & -2707.31933 & -2707.33991 \\
\hline $\mathrm{H}_{2} \mathrm{O}-\mathrm{H}^{+}$ & -76.59898 & -76.32800 & -210.36996 & -210.39010 \\
\hline $\mathrm{HCN}-\mathrm{H}^{+}$ & -93.53583 & -93.25893 & -227.30336 & -227.32273 \\
\hline $\mathrm{HNC}-\mathrm{H}^{+}$ & -93.53583 & -93.23060 & -227.27651 & -227.29561 \\
\hline $\mathrm{LiCl}-\mathrm{H}^{+}$ & -467.57582 & -467.25865 & -601.32189 & -601.34039 \\
\hline $\mathrm{H}_{3} \mathrm{~N}-\mathrm{H}^{+}$ & -56.79500 & -56.45925 & -190.50907 & \\
\hline $\mathrm{MeH}_{2} \mathrm{~N}-\mathrm{H}^{+}$ & -96.01892 & -95.66532 & -229.72906 & \\
\hline $\mathrm{LiCN}-\mathrm{H}^{+}$ & -100.52428 & -100.16258 & -234.24255 & -234.22640 \\
\hline $\mathrm{Me}_{2} \mathrm{HN}-\mathrm{H}^{+}$ & -135.24405 & -134.87768 & -268.95197 & -268.94832 \\
\hline $\mathrm{Me}_{3} \mathrm{~N}-\mathrm{H}^{+}$ & -174.47027 & -174.09765 & -308.17683 & -308.17408 \\
\hline $\mathrm{NaCN}-\mathrm{H}^{+}$ & -254.94716 & -254.56709 & -388.65651 & -388.64603 \\
\hline $\mathrm{LiNC}_{-} \mathrm{H}^{+}$ & -100.55123 & -100.16324 & -234.25017 & -234.24650 \\
\hline $\mathrm{Br}-\mathrm{H}$ & -2573.29360 & -2572.77480 & -2706.98041 & -2706.97819 \\
\hline $\mathrm{Cl}-\mathrm{H}$ & -460.31445 & -459.78079 & -594.00120 & -593.99864 \\
\hline $\mathrm{CN}-\mathrm{H}$ & -93.23060 & -92.69289 & -226.92073 & -226.91714 \\
\hline $\mathrm{NC}-\mathrm{H}$ & -93.25893 & -92.69289 & -226.94254 & -226.94110 \\
\hline $\mathrm{F}-\mathrm{H}$ & -100.34038 & -99.74588 & -234.03157 & -234.02685 \\
\hline
\end{tabular}


Table S5. MP2/aug'-cc-pVTZ Energies (hartrees) for the Hydrogen-Bonded Complexes of Furan with A-H ${ }^{\mathrm{n}+}$

\begin{tabular}{|c|c|c|c|}
\hline \multirow[t]{2}{*}{$\mathrm{A}-\mathrm{H}^{\mathrm{n}+}$} & \multicolumn{3}{|c|}{$\mathrm{A}-\mathrm{H}^{\mathrm{n}+}$-furan complex } \\
\hline & 1 & 2 & 3 \\
\hline $\mathrm{HF}-\mathrm{H}^{+}$ & -330.22430 & -330.25598 & -330.23732 \\
\hline $\mathrm{HCl}-\mathrm{H}^{+}$ & -690.19496 & -690.22601 & -690.20751 \\
\hline $\mathrm{HBr}-\mathrm{H}^{+}$ & -2803.17629 & -2803.20590 & -2803.18486 \\
\hline $\mathrm{H}_{2} \mathrm{O}-\mathrm{H}^{+}$ & -306.23670 & -306.24938 & -306.23058 \\
\hline $\mathrm{HCN}-\mathrm{H}^{+}$ & -323.16847 & -323.18043 & -323.16336 \\
\hline $\mathrm{HNC}-\mathrm{H}^{+}$ & -323.15286 & & -323.15920 \\
\hline $\mathrm{H}_{3} \mathrm{~N}-\mathrm{H}^{+}$ & -286.41069 & & -286.41305 \\
\hline $\mathrm{LiCN}-\mathrm{H}^{+}$ & -330.13763 & & -330.13655 \\
\hline $\mathrm{NaCN}-\mathrm{H}^{+}$ & -484.55801 & & -484.55705 \\
\hline${\mathrm{LiNC}-\mathrm{H}^{+}}^{+}$ & -330.15674 & & -330.15996 \\
\hline $\mathrm{Br}-\mathrm{H}$ & -2802.89140 & & -2802.89262 \\
\hline $\mathrm{Cl}-\mathrm{H}$ & -689.91223 & & -689.91269 \\
\hline $\mathrm{CN}-\mathrm{H}$ & -322.83138 & & -322.83117 \\
\hline $\mathrm{NC}-\mathrm{H}$ & -322.85549 & & -322.85719 \\
\hline $\mathrm{F}-\mathrm{H}$ & -329.94114 & & -329.93911 \\
\hline
\end{tabular}

\section{Full Reference}

(16) Frisch, M. J.; Trucks, G. W.; Schlegel, H. B.; Scuseria, G. E.; Robb, M. A.; Cheeseman, J. R.; Montgomery, J. A., Jr.; Vreven, T.; Kudin, K. N.; Burant, J. C.; Millam, J. M.; Iyengar, S. S.; Tomasi, J.; Barone, V.; Mennucci, B.; Cossi, M.; Scalmani, G.; Rega, N.; Petersson, G. A.; Nakatsuji, H.; Hada, M.; Ehara, M.; Toyota, K.; Fukuda, R.; Hasegawa, J.; Ishida, M.; Nakajima, T.; Honda, Y.; Kitao, O.; Nakai, H.; Klene, M.; Li, X.; Knox, J. E.; Hratchian, H. P.; Cross, J. B.; Adamo, C.; Jaramillo, J.; Gomperts, R.; Stratmann, R. E.; Yazyev, O.; Austin, A. J.; Cammi, R.; Pomelli, C.; Ochterski, J. W.; Ayala, P. Y.; Morokuma, K.; Voth, G. A.; Salvador, P.; Dannenberg, J. J.; Zakrzewski, V. G.; Dapprich, S.; Daniels, A. D.; Strain, M. C.; Farkas, O.; Malick, D. K.; Rabuck, A. D.; Raghavachari, K.; Foresman, J. B.; Ortiz, J. V.; Cui, Q.; Baboul, A. G.; Clifford, S.; Cioslowski, J.; Stefanov, B. B.; Liu, G.; Liashenko, A.; Piskorz, P.; Komaromi, I.; Martin, R. L.; Fox, D. J.; Keith, T.; Al-Laham, M. A.; Peng, C. Y.; Nanayakkara, A.; Challacombe, M.; Gill, P. M. W.; Johnson, B.; Chen, W.; Wong, M. W.; Gonzalez, C.; Pople, J. A. Gaussian 03, revision B.03; Gaussian, Inc.: Pittsburgh, PA, 2003 